\title{
GROWTH IN GROUPS: IDEAS AND PERSPECTIVES
}

\author{
HARALD A. HELFGOTT \\ In memory of Ákos Seress (1958-2013)
}

\begin{abstract}
This is a survey of methods developed in the last few years to prove results on growth in non-commutative groups. These techniques have their roots in both additive combinatorics and group theory, as well as other fields. We discuss linear algebraic groups, with $\mathrm{SL}_{2}(\mathbb{Z} / p \mathbb{Z})$ as the basic example, as well as permutation groups. The emphasis will lie on the ideas behind the methods.
\end{abstract}

\section{INTRODUCTION}

The study of growth and expansion in infinite families of groups has undergone remarkable developments in the last decade. We will see some basic themes at work in different contexts - linear groups, permutation groups - which have seen the conjunction of a variety of ideas, including combinatorial, geometric, and probabilistic. The effect has been a still-ongoing series of results that are far stronger and more general than those known before.

1.1. What do we mean by "growth"? Let $A$ be a finite subset of a group $G$. Consider the sets

$A$,

$$
\begin{aligned}
A \cdot A & =\{x \cdot y: x, y \in A\}, \\
A \cdot A \cdot A & =\{x \cdot y \cdot z: x, y, z \in A\}, \\
& \cdots \\
A^{k} & =\left\{x_{1} x_{2} \ldots x_{k}: x_{i} \in A\right\} .
\end{aligned}
$$

Write $|S|$ for the size of a finite set $S$, meaning simply the number of elements of $S$. A question arises naturally: how does $\left|A^{k}\right|$ grow as $k$ grows?

This is just one of a web of interrelated questions that, until recently, were studied within separate areas of mathematics:

- additive combinatorics treats the case of $G$ abelian,

- geometric group theory studies $\left|A^{k}\right|$ as $k \rightarrow \infty$ for $G$ infinite,

- subgroup classification, within group theory, can be said to study the special case $|A|=|A \cdot A|$, since, for $A$ finite with $e \in A$, we have $|A|=|A \cdot A|$ precisely when $A$ is a subgroup of $G$. (As we shall later see, this is not a joke, or rather it is a very fruitful one.)

Received by the editors March 11, 2013, and, in revised form, September 24, 2013, and July 30, 2014.

2010 Mathematics Subject Classification. Primary 20F69; Secondary 20D60, 11B30, 20 B05. 
When $G$ is finite, rather than asking ourselves how $\left|A^{k}\right|$ behaves for $k \rightarrow \infty$-it obviously becomes constant as soon as $A^{k}=\langle A\rangle$, where $\langle A\rangle$ is the subgroup of $G$ generated by $A$-we ask what is the least $k$ such that $A^{k}=G$. (Here and henceforth we assume that $A$ does generate $G$, and that $e \in A$.) This value of $k$ is called the diameter of $G$ with respect to $A$. The word "diameter" comes from graph theory; as we shall see, a pair $(G, A)$ has a graph associated to it, and the diameter of the graph is the same as the diameter we have just defined.

Other key related concepts are:

- expander graphs: every expander graph has very small diameter (where very small $=O(\log |G|))$, but not vice versa - that is, expansion is stronger than having very small diameter;

- mixing time, meaning the least $k$ such that, when $x_{1}, \ldots, x_{k}$ are taken uniformly and at random within $A$, the distribution of the product $x_{1} \cdots x_{k}$ is close to uniform.

We will make both notions precise later.

This survey covers a series of rapid developments on these questions since 2005 . The focus will be on $G$ finite. Some of the results here extend to infinite groups with ease; indeed, working with, say, matrix groups over $\mathbb{Z} / p \mathbb{Z}$ is often strictly harder than working over matrix groups over $\mathbb{R}$ or $\mathbb{C}$. The emphasis will be on methodsoften with a distinct combinatorial flavor - that made strong, general results on finite groups possible.

Several main themes run through proofs that seem very different on the technical surface. One of them is the idea of stable configurations under group actions as the main object of study. It is this, with slowly growing sets (approximate subgroups) as a special case, that encapsulates not just the results but a great deal of the approach to proving them.

Contributions have been made by mathematicians from many fields. While one cannot claim or attempt completeness, I have tried to give as full a historical picture as I have been able to; this is so in part to give credit to all those to whom credit is due, but also so as to familiarize readers who are experts in a field with work coming from other domains, and most of all so as to make both the ideas themselves and their actual development clear.

1.2. Main results covered. Our focus will lie on the ideas behind the main growth results, going from $\mathrm{SL}_{2}(\mathbb{Z} / p \mathbb{Z})$ ([Hel08], reexamined in the light of [Hel11], [BGT11, PSa]) up to new work on the symmetric group [HS14. We will be able to give essentially complete proofs of Theorems 1.11 .4 below.

In particular, this includes Theorem 1.3 (Bourgain and Gamburd), the result that set a paradigm on how to prove expansion starting from a result on growth in groups. We will spend less time on the applications of expansion; while that is undoubtedly a rich topic, it is one whose developments to date have been well covered in Kowb and Lub12. The notes Kowa and Tao treat some of the material in the present survey as well as some applications.

1.2.1. Growth in linear groups. One of our goals will be to show the main ideas behind proofs of growth in linear groups of bounded rank. In particular, we will give most of the details of what amounts to an "up-to-date" proof of the following result in such a way that the proof generalizes naturally. (In other words, it will incorporate ideas from the series of developments to which the first proof gave rise.) 
Theorem 1.1 (Helfgott $\left[\right.$ Hel08]). Let $G=\mathrm{SL}_{2}\left(\mathbb{F}_{p}\right)$. Let $A \subset G$ generate $G$. Then either

$$
\left|A^{3}\right| \geq|A|^{1+\delta}
$$

or $\left(A \cup A^{-1} \cup\{e\}\right)^{k}=G$, where $\delta>0$ and $k \geq 1$ are absolute constants.

Here, as usual, "absolute constant" means "really a constant"; in particular, the constants $\delta$ and $k$ do not depend on $p$. Nikolov and Pyber [NP11] (following Gowers Gow08; see also BNP08) showed that one can replace $\left(A \cup A^{-1} \cup\{e\}\right)^{k}$ by $A^{3}$. Kowalski Kow13 has shown one can take $\delta=1 / 3024$ (assuming $A=A^{-1}$, $e \in A$; we will see in 3.1 that these are very "light" assumptions). On the other end, Button and Roney-Dougal [BRD] have given an example that shows that $\delta \leq\left(\log _{2} 7-1\right) / 6=0.30122 \ldots$

There are two main kinds of generalizations: changing the field and changing the Lie type. We will discuss them at the end of \$5.4. In brief, Theorem 1.1 is now known to hold (with $\delta$ depending on the rank) for all finite simple groups of Lie type ([PSa, [BGT11]); there are also generalizations to $\mathbb{C}$ [BG08b] and to solvable groups $\mathrm{GH}$.

Corollary 1.2. The diameter of $G_{p}=\mathrm{SL}_{2}(\mathbb{Z} / p \mathbb{Z})$ with respect to any set of generators $A$ is $\left(\log \left|G_{p}\right|\right)^{O(1)}$.

The proof of this Corollary to Theorem 1.1 is really a simple exercise; this is a good point for the reader to grab a nearby pencil.

Proof. Applying Theorem $1.1 \ell$ times, we obtain that either

$$
\left|A^{3^{\ell}}\right| \geq|A|^{(1+\delta)^{\ell}}
$$

or

$$
\left(S \cup S^{-1} \cup\{e\}\right)^{k}=G_{p}
$$

for $S=A^{3^{\ell-1}}$. We choose $\ell=\left\lfloor\left(\log \left(\log \left|G_{p}\right| / \log |A|\right)\right) / \log (1+\delta)+1\right\rfloor$, which, if (1.1) were true, would give us $\left|A^{3^{\ell}}\right|>|G|$; since this is absurd, (1.2) holds, and so the diameter of $G_{p}$ with respect to $A \cup A^{-1}$ is at most

$$
\ll k\left(\log \left|G_{p}\right| / \log |A|\right)^{\frac{1}{\log 1+\delta}} \ll\left(\log \left|G_{p}\right|\right)^{O(1 / \delta)} .
$$

Now, a general result Bab06, Thm. 1.4] states that the diameter $d^{+}$of a group $G$ with respect to a set of generators $A$ is at most $d^{2}(\log |G|)^{3}$, where $d$ is the diameter of $G$ with respect to the set of generators $A \cup A^{-1}$. Thus, (1.3) implies that the diameter of $G_{p}$ with respect to $A$ is

$$
\ll\left(\log \left|G_{p}\right|\right)^{O(2 / \delta)+3}=\left(\log \left|G_{p}\right|\right)^{O(1 / \delta)} .
$$

(We could have avoided the use of Bab06, Thm. 1.4] by applying the improved version of Theorem 1.1, with $A^{3}=G$ instead of $\left(A \cup A^{-1} \cup\{e\}\right)^{k}$, but why avoid quoting an elegant general result that the reader could find useful?) Since $\delta$ is an absolute constant and $\left|G_{p}\right| \geq 6$, (1.4) bounds the diameter by $\left(\log \left|G_{p}\right|\right)^{O(1)}$, as was desired.

A conjecture due to Babai (see [BS88, p. 176]) states that, for any finite, nonabelian simple group $G$ and any set of generators $A$, the diameter of $G$ with respect to $A$ is $O\left((\log |G|)^{C}\right)$, where $C$ is an absolute constant. Thus, Corollary 1.2 states that Babai's conjecture holds for $G=\mathrm{SL}_{2}(\mathbb{Z} / p \mathbb{Z})$ and $A$ arbitrary. (We should 
actually say: "for $G=\operatorname{PSL}_{2}(\mathbb{Z} / p \mathbb{Z})$ ", since $\mathrm{SL}_{2}$ is just almost simple, whereas $\mathrm{PSL}_{2}$ really is simple.)

Consider now an important special case: let $A$ be a subset of $\mathrm{SL}_{2}(\mathbb{Z})$, and suppose that its projections $A_{p}=A \bmod p$ generate $G_{p}$ for $p$ larger than a constant. This is the case, in particular, if $\langle A\rangle$ is Zariski-dense, i.e., if it is not contained in a proper subvariety of $\mathrm{SL}_{2}$. (The implication is proven, in varying degrees of generality, in Wei84, MVW84, Nor87, and [HP95.) Both (2.1) and (2.2) are examples of valid $A$.

It is not hard to show (as we will sketch in 5.5 ) that, in this situation, Theorem 1.1 implies a logarithmic bound $\left(O_{A}(\log |G|)\right)$ for the diameter of $G_{p}$ with respect to $A_{p}$. Bourgain and Gamburd proved a rather stronger statement.

Theorem 1.3 (Bourgain and Gamburd $[\mathrm{BG08c})$. Let $A \subset \mathrm{SL}_{2}(\mathbb{Z})$ generate a Zariski-dense subgroup of $\mathrm{SL}_{2}(\mathbb{Z})$. Then $\left(\mathrm{SL}_{2}(\mathbb{Z} / p \mathbb{Z}), A \bmod p\right)$ are a family of expanders.

In general, expansion is stronger than logarithmic mixing time, which is stronger than logarithmic diameter. Bourgain and Gamburd first show that a kind of mixing time (in a very weak sense, an $\ell$ such that $\left|\mu^{(\ell)}\right|_{2}^{2} \ll|G|^{-1+\epsilon}$, where $\mu^{(\ell)}$ is the distribution after $\ell$ steps of a random walk) is indeed logarithmic: For the first $\log _{c}(p / 2)$ steps, a random walk mixes well (for the same reason as what we said we will sketch in $\$ 5.5)$. Then, for a constant number of steps, they apply a result on the $\ell_{2}$ "flattening" of measures under convolutions (Proposition 3.5) that they prove using Theorem 1.1 (via a non-commutative version [Tao08] of a result from additive combinatorics, the Balog-Szemerédi-Gowers theorem). The fact that expansion does follow from a logarithmic bound on this kind of weak mixing time for groups, such as $\mathrm{SL}_{2}(\mathbb{Z} / p \mathbb{Z})$, is due to Sarnak and Xue [SX91]. We will go over this in detail in $\$ 5.5$.

Theorem 1.3 has found manifold applications (see, e.g., BGS10, the affine sieve); we refer again to Kowb and Lub12. Traditionally, such results as we had on expansion in $\mathrm{SL}_{2}(\mathbb{Z} / p \mathbb{Z})$ were deduced from results on the spectral gap of the (continuous) Laplacian on the surface $\Gamma(p) \backslash \mathbb{H}$. Theorem 1.3 is a much more general result, based on a combinatorial result, namely, Theorem 1.1.

Later, Bourgain, Gamburd, and Sarnak BGS11 reversed the traditional direction of the implication, showing that Theorem 1.3 can be used to obtain spectral gaps for the Laplacian on general quotients $\Lambda \backslash \mathbb{H}\left(\Lambda<\mathrm{SL}_{2}(\mathbb{Z})\right.$ Zariski-dense in $\left.\mathrm{SL}_{2}\right)$.

By now, as we shall see in \$5.4 Theorems 1.1 and 1.3 have been generalized several times. There remain two open questions, both related to uniformity. One is whether $\left(\mathrm{SL}_{2}(\mathbb{Z} / p \mathbb{Z}), A_{p}\right)$ might be a family of expanders as $A_{p}$ ranges over all generating sets $A_{p} \subset \mathrm{SL}_{2}(\mathbb{Z} / p \mathbb{Z})$. The other one concerns uniformity on the rank of the group. Theorem 1.1 is now known to hold over $\mathrm{SL}_{n}$, which is of rank $n-1$, but only if $\delta$ is allowed to depend on $n$. If $\delta>0$ is held constant and $n \rightarrow \infty$, counterexamples are known. The question is then whether consequences such as Corollary 1.2 might still be true uniformly, i.e., with implied constants independent of $n$.

1.2.2. The symmetric group and beyond. The Classification of Finite Simple Groups tells us that every finite, simple, non-abelian group is either a matrix group, or the alternating group $\operatorname{Alt}(n)$, or one of a finite list of exceptions. (The list is irrelevant for asymptotic statements precisely because it is finite.) All of the above work 
on matrix groups leaves unanswered the corresponding questions on diameter and growth in $\operatorname{Alt}(n)$ and other permutation groups.

The question of the diameter of permutation groups can be stated precisely in a playful way. Let a set $A$ of ways to scramble a finite set $\Omega$ be given. This is the familiar setting of permutation puzzles: Rubik's cube, Alexander's star, Hungarian rings.... People say that a position has a solution if it can be unscrambled back to a fixed "starting position" by means of some succession of moves in $A$. Given that we are told that a position has a solution, does it follow that it has a short solution?

The answer is yes HS14. The only condition is that $\langle A\rangle$ be transitive, i.e., that, given two elements $x, y$ of $\Omega$, there be a succession of moves in $A$ that, when combined, take $x$ to $y$. Transitivity is necessary: it is easy to construct a non-transitive group of very large diameter [BS92, Example 1.2]. (However, if the number of orbit 1 is bounded, then the problem reduces to the transitive case.)

Thinking for a moment, we see that a request for short solutions is the same as a request for a small diameter: if $A=A^{-1}$, the diameter of $\langle A\rangle$ with respect to $A$ equals the maximum, over all positions, of the length of the shortest solution to that position.

As BS92 showed, questions on the diameter of permutation groups reduce (with some loss) to the case $G=\operatorname{Alt}(n)$. Babai's conjecture gives that the diameter of $\operatorname{Alt}(n)$ with respect to any set of generators is $\ll(\log |G|)^{O(1)} \ll n^{O(1)}$. This special case of the conjecture actually predates Babai; Babai and Seress [BS92, p. 232] call it "folkloric". There are earlier references in print KMS84, McK84 where the question is posed as to the exact conditions under which a bound of $n^{O(1)}$ might be valid; in particular ( KMS84, §4]), might transitivity be sufficient? For an arbitrary transitive subgroup of $\operatorname{Sym}(n)$, Babai and Seress [BS92, Conj. 1.6] conjectured the weaker bound

$$
\leq \exp \left((\log n)^{O(1)}\right)
$$

on the diameter with respect to any set of generators.

Theorem 1.4 (Helfgott and Seress [HS14). Let $G=\operatorname{Sym}(n)$ or $\operatorname{Alt}(n)$. Let $A$ be any set of generators of $G$. Then the diameter of $G$ with respect to $A$ is

$$
\leq \exp \left(O\left((\log n)^{4} \log \log n\right)\right),
$$

where the implied constant is absolute.

By a general result BS92] bounding the diameter of a transitive group in terms of the diameter of its largest factor of the form $\operatorname{Alt}(n)$, Theorem 1.4 implies that (1.5) holds for all transitive permutation groups on $n$ elements and any set of generators.

What is the importance of $\operatorname{Sym}(n)$ and $\operatorname{Alt}(n)$, from the perspective of linear groups? It is not just a matter of historical importance (in that conjectures for permutation groups preceded Babai's more general conjecture) or of generality. The groups $\operatorname{Sym}(n)$ and $\operatorname{Alt}(n)$ are, in a sense, creatures of pure rank; $\operatorname{Sym}(n)$ corresponds particularly closely to what $\mathrm{SL}_{n}$ over a field with one element would be like 2 Uniformity on the rank is precisely what is still missing in the linear algebraic

\footnotetext{
${ }^{1}$ An orbit, in a permutation group $G<\operatorname{Sym}(n)$, means an orbit of $\{1,2, \ldots, n\}$ under the action of $G$. Thus, Rubik's cube has three orbits: corners, sides, and centers (if you are allowed to rotate the cube in space).

${ }^{2}$ While the field with one element does not exist, objects over the field with one element can be defined and studied. This is an idea going back to Tits [Tit57]; see, e.g., [Lor12].
} 
case; the new result on $\operatorname{Sym}(n)$ and $\operatorname{Alt}(n)$ can be seen - optimistically — as breaking the barrier of rank dependence, just as Hel08 showed that independence on the field was a feasible goal.

In parallel to the work on permutation groups in the line of BLS87, BBS04, et al.- works having their roots in the study of algorithms - there is also an entire related area of study coming from probability theory. This area is well represented by the text LPW09; the emphasis there is in part on mixing times for random processes that may be more general than a random walk. See, for example, results expressed in terms of card-shuffling, such as the Bayer-Diaconis "seven-shuffle" theorem [BD92].

The interest in studying the diameter and the expander properties of $\langle A\rangle$ with respect to $A$ for $A=\{g, h\} \subset \operatorname{Sym}(n), g, h$ random, comes in part from this area. (This is also of interest for linear algebraic groups; see BG08c.) Here a result of Babai and Hayes [BH05] based on BBS04 shows that, almost certainly (i.e., with probability tending to 1 as $n \rightarrow \infty)$, the diameter of $\langle A\rangle$ with respect to $A$ is polynomial in $n$. (A classical result of Dixon Dix69 states that $\langle A\rangle$ is almost certainly $\operatorname{Sym}(n)$ or $\operatorname{Alt}(n)$.) Schlage and Puchta SP12] improved the bound to $O\left(n^{3} \log n\right)$.

Recent work HSZ proves that the diameter of $\langle A\rangle$ with respect to $A$ is in fact $n^{2}(\log n)^{O(1)}$ with probability tending to 1 as $n \rightarrow \infty$; the mixing time is $n^{3}(\log n)^{O(1)}$. At play is a combination of probabilistic and combinatorial ideas ( $\mathrm{BS} 87 \mathrm{~b}$, BBS04]); there is some common ground with the ideas in [HS14] (discussed here in 6 on generation and random walks.

1.3. Notation. By $f(n) \ll g(n), g(n) \gg f(n)$ and $f(n)=O(g(n))$, we mean the same thing, namely, that there are $N>0, C>0$ such that $|f(n)| \leq C \cdot g(n)$ for all $n \geq N$. We write $\ll_{a}, \gg_{a}, O_{a}$ if $N$ and $C$ depend on $a$ (say).

As usual, $f(n)=o(g(n))$ means that $|f(n)| / g(n)$ tends to 0 as $n \rightarrow \infty$. We write $O^{*}(x)$ to mean any quantity at most $x$ in absolute value. Thus, if $f(n)=O^{*}(g(n))$, then $f(n)=O(g(n))$ (with $N=1$ and $C=1$ ).

Given a subset $A \subset X$, we let $1_{A}: G \rightarrow \mathbb{C}$ be the characteristic function of $A$ :

$$
1_{A}(x)= \begin{cases}1 & \text { if } x \in A, \\ 0 & \text { otherwise. }\end{cases}
$$

\section{Cultural Background}

The problems addressed in this survey first arose and were studied within different areas. The purpose of this section is to give a glimpse of a broad picture, or rather a series of glimpses from different perspectives. For the sake of clarity, and to avoid repetitions with earlier and later discussions of recent results, we will look at matters as they stood in different fields right before Theorem 1.1 was proven, followed quickly by Theorem 1.3 and a series of results by many authors.

This section is, so to speak, a "cultural" one, in that it presents matters quickly, without any claim to completeness, with the aim of setting a scene; it could in principle be skipped at first by a reader in a hurry. While it does define a key concept - that of expansion - and it makes more precise another one - the notion of mixing time - we will review the definitions later, before their first technical use. 
What would be missed by skipping this section would mainly be motivation, and a sense of the newness of some of the interactions between the fields presented here as separate units.

2.1. Additive combinatorics. This is a relatively recent name for a field one of whose starting points is the work of Freiman ([Fre73; see also Ruz91]) classifying subsets $A$ of $\mathbb{Z}$ such that $A+A$ is not much larger than $A$. (The strongest bound to date is that of Sanders [San12]; we still do not have the strongest conjectured form of this kind of result.) Work by Ruzsa and others ([Plü70, Ruz89, [RT85]) showed how that, if $|A+A|$ is not much larger than $|A|$, then the sequence $|A|,|A+A|,|A+A+A|, \ldots$ grows slowly; we will go over this in 3.1

The use of the sign "+" and the word "additive" both remind us that additive combinatorics treated abelian groups, even if some of its techniques turned out to generalize to non-abelian groups rather naturally (see, e.g., [Tao08]). We will take a detailed look at some of the bases of the subject in $\$ 3$

2.2. Expanders, spectral gaps, and property ( $\mathbf{T})$. Let us start by defining expanders. For $A$ a set of generators of a finite group $G$, we say that the pair $(G, A)$ gives us an $\epsilon$-expander if every subset $S$ of $G$ with $|S| \leq|G| / 2$ satisfies $|S \cup A S| \geq(1+\epsilon)|S|$. An $\epsilon$-expander always has very small diameter (meaning diameter $=O((\log |G|) / \epsilon))$ and, if the identity $e$ is in $A$, it also has very small $\ell_{2}$ and $\ell_{\infty}$ mixing time.

Alternatively, we can define the expander property in terms of the size of the second smallest eigenvalue $\lambda_{1}$ of the discrete Laplacian of the Cayley graph. The (normalized) adjacency matrix $\mathscr{A}$ of a graph is the operator that maps a function $f$ on the set of vertices $V$ of the graph to the function $\mathscr{A} f$ on $V$ whose value at $v$ is the average of $f(w)$ on the neighbors $w$ of $v$. The discrete Laplacian is simply $\triangle=I-\mathscr{A}$. A Cayley graph $\Gamma(G, A)$ is the graph having $G$ as its set of vertices and $\{(g, a g): g \in G, a \in A\}$ as its set of edges. Assume $A=A^{-1}$; then $\triangle$ is a symmetric operator, and all its eigenvalues $\lambda_{0}, \lambda_{1}, \ldots$ are thus real. Clearly, the smallest eigenvalue is $\lambda_{0}=0$, corresponding to the constant eigenfunctions. We say that $\Gamma(G, A)$ is an $\epsilon$-expander if $\lambda \geq \epsilon$ for every eigenvalue $\lambda$ corresponding to a non-constant eigenfunction. For $|A|$ bounded, this is equivalent to the first definition we gave of an $\epsilon$-expander (though the constant $\epsilon$ is different in the two sides of the equivalence).

We can order the eigenvalues $0=\lambda_{0} \leq \lambda_{1} \leq \lambda_{2} \leq \cdots$. We call $\left|\lambda_{1}-\lambda_{0}\right|=\lambda_{1}$ the spectral gap. We say that a family of graphs is an expander family, or has a spectral gap, if there is an $\epsilon>0$ such that, for every graph $\Gamma$ in the family, $\Gamma$ is an $\epsilon$-expander.

Of course, the discrete Laplacian is an analogue of the classical Laplacian $\Delta$, defined on surfaces. The Laplacian $\Delta$ on a surface also has real, non-negative eigenvalues; we speak of a spectral gap if there is an $\epsilon>0$ such that $\lambda>\epsilon$ for every eigenvalue $\lambda$ corresponding to a non-constant eigenfunction of $\Delta$. An especially well-researched class of surfaces is that given by quotients $G \backslash \mathbb{H}$ of the upper halfplane $\mathbb{H}=\{x+i y: y>0\}$ by subgroups $G$ of $\mathrm{SL}_{2}(\mathbb{R})$ : the group $\mathrm{SL}_{2}(\mathbb{R})$ acts on $\mathbb{H}$ by fractional linear transformations, and thus, given a discrete subgroup $G$ of $\mathrm{SL}_{2}(\mathbb{R})$, we can study the surface $G \backslash \mathbb{H}$, which is the result of identifying points $x_{1}, x_{2}$ of $\mathbb{H}$ for which there is a $g \in G$ such that $g\left(x_{1}\right)=x_{2}$. In particular, consider the discrete subgroup $\Gamma(N)=\left\{g \in \mathrm{SL}_{2}(\mathbb{Z}): g \equiv I \bmod N\right\}$. A central 
result in the theory of modular forms (Selberg [Sel65]) states that the Laplacian on the surface $\Gamma(N) \backslash \mathbb{H}$ has a spectral gap. It has long been known (see 95.5 ) that the existence of this spectral gap on the surface $\Gamma(N) \backslash \mathbb{H}$ implies that the pairs $\left(\mathrm{SL}_{2}(\mathbb{Z} / p \mathbb{Z}), A \bmod p\right)$ with

$$
A=\left\{\left(\begin{array}{ll}
1 & 1 \\
0 & 1
\end{array}\right),\left(\begin{array}{ll}
1 & 0 \\
1 & 1
\end{array}\right)\right\}
$$

are an expander family, i.e., are $\epsilon$-expanders for some fixed $\epsilon>0$ as $p$ varies over all primes. Before [Hel08] and [BG08c, little was known for more general A; e.g., for

$$
A=\left\{\left(\begin{array}{ll}
1 & 3 \\
0 & 1
\end{array}\right),\left(\begin{array}{ll}
1 & 0 \\
3 & 1
\end{array}\right)\right\}
$$

there were no good diameter bounds, let alone a proof that $\left(\mathrm{SL}_{2}(\mathbb{Z} / p \mathbb{Z}), A \bmod p\right)$ is a family of expanders. (This is a favorite example of Lubotzky's.)

For $G=\mathrm{SL}_{n}(\mathbb{Z} / p \mathbb{Z}), n \geq 3$, the proof of expansion for some $A$ was arguably more direct (due to property $(\mathrm{T})$, for which relatively elementary proofs were known Kaž67]), but the case of general $A$ was open, just as for $n=2$.

Kassabov applied what was known for $\mathrm{SL}_{n}$ (and linear algebraic groups in general) to prove the existence of expanders for the symmetric group Kas07. Other relevant works are [SX91] (giving an elementary treatment of expansion for $A$ as in (2.1), and, in general, "arithmetic lattices" 3 in $\mathrm{SL}_{2}$ ), Sha97, Sha99, Gam02 (strengthening and generalizing [SX91] to some infinite-index groups), and GS04] and Din06 (both of them influenced by the Solovay-Kitaev algorithm, as in [NC00, App. 3]; see [Var for recent work along this line). Still, (2.2) remained a frustrating example of wide classes of generators for which little was known.

2.3. Mixing times and diameters. Let $A$ be a set of generators of a finite group $G$. Recall that the diameter of $G$ with respect to $A$ is defined to be the least $k$ such that every element $g$ of $G$ can be written as $g=x_{1} x_{2} \cdots x_{r}$ for some $x_{i} \in A, r \leq k$; this is the same as the graph-theoretical diameter of the Cayley graph $\Gamma(G, A)$. As we said before, Babai's conjecture [BS88, p. 176] posits that, if $G$ is simple and non-abelian, the diameter of $G$ with respect to any set $A$ of generators is small, that is to say, $(\log |G|)^{O(1)}$. (Note that the bound does not depend on $A$.) For the alternating group $G=\operatorname{Alt}(n)$, this was a folklore conjecture; work toward it includes [BS88], BKL89], and BBS04].

The mixing time is the least $k$ such that, when $x_{1}, \ldots, x_{k}$ are taken uniformly and at random within $A$, the distribution of the product $x_{1} \cdots x_{k}$ (or, if you prefer, the outcome of a random walk of length $k$ on the Cayley graph $\Gamma(G, A))$ is close to uniform in $G$. We speak of $\ell_{2}$ mixing time, $\ell_{\infty}$ mixing time, etc., depending on the norm used to define "close to". Here most work has focused on permutation groups, with a strong probabilistic flavor; see [BBS04, BH05], [BH92, DS81, [DSC93], as well as the recent, rather thorough source [LPW09], which contains plenty of references.

\footnotetext{
${ }^{3}$ For more on the case of arithmetic lattices (a case that covers (2.1) but not (2.2), see the references in GV12, §1.1].
} 
2.4. Group theory: subgroup classification. If $e \in A$, the extreme case $|A \cdot A|=|A|$ happens exactly when $A$ is a subgroup of $G$. There are results on subgroup classification from the 1980s and 1990s intended to bypass parts of the Classification of Finite Simple Groups by elementary arguments. The motivation was clear: the Classification Theorem had - especially at first - an extremely long proof, dispersed in many articles. Thus, alternative proofs for some of its consequences were in demand.

As we will later see, several of these alternative, relatively elementary arguments - in particular, those in LP11] (circulated in preprint form since ca. 1998), Bab82 and Pyb93 - later played an important role in the study of growth: their techniques for studying sets with $|A \cdot A|=|A|$ (that is, subgroups) turned out to be robust enough to extend to the study of sets for which $|A \cdot A|$ is not much larger than $|A|$.

2.5. Asymptotic group theory. Model theory. As we said in 1.1 geometric group theory tends to study the growth of $\left|A^{k}\right|$ for $k \rightarrow \infty$, where $A$ is a set of generators of an infinite group $G$. One of the main results of geometric group theory is Gromov's theorem [Gro81], which states that if $\left|A^{k}\right| \ll k^{O(1)}$, then $G$ is virtually nilpotent, i.e., $G$ has a nilpotent subgroup of finite index. In other words, Gromov's theorem classifies groups where $A^{k}$ grows very slowly (meaning polynomially).

Model theory is essentially a branch of logic with applications to algebraic structures. The work of Hrushovski and his collaborators, culminating in Hru12 (see also [HP95] and [HW08]), used model theory to study subgroups of algebraic groups. (As we will see later, this effort was influenced by the work of Larsen and Pink [LP11] and also served to elucidate it.) In Hru12, Hrushovski gave a new proof of Gromov's theorem.

We will later have the chance to discuss (\$5.2) the recent influence of [HP95], [HW08, and [Hru12] on the area of this survey and on the study of growth in finite linear algebraic groups in particular.

\section{Methodological BaCKGRound: ARITHMetic COMBinatorics}

The terms "additive combinatorics" and "arithmetic combinatorics" are relatively new. To judge from [TV06], they cover at least some of additive number theory and the geometry of numbers. What may be called the core of additive combinatorics is the study of the behavior of arbitrary sets under addition (as opposed to, say, the primes or $k$ th powers). In this sense, the subject originated from at least two streams, one coursing through work on arithmetic progressions by Schur, van der Waerden, Roth [Rot53, Szemerédi [Sze69], Furstenberg [Fur77] (leading to the ergodic work of Host and Kra HK05] and Ziegler [Zie07]; see also Szegedy [Sze], Gowers Gow01, and Green and Tao GT08, among many others, and another one based on the study of growth in abelian groups, starting with Freiman [Fre73, Erdős and Szemerédi [ES83, and Ruzsa [Ruz94], Ruz99] and continuing in GR05, Cha02, San12. There has also been a vein of a more geometrical flavor (e.g., ST83]).

The use of the term arithmetic combinatorics instead of additive combinatorics emphasizes results on growth that do not require commutativity, as well as results on fields and rings (the sum-product theorem, \$3.3).

The reader who is playing hopscotch may jump ahead to 44 and thus miss the following material: the reason why Theorem 1.1 is and can be stated as a 
lower bound on $\left|A^{3}\right|$ (\$3.1), the connection of such a bound to the definition of approximate subgroups (also \$3.1), the Balog-Szemerédi-Gowers theorem and its use by Bourgain and Gamburd to establish an equivalence between the growth of sets and the flattening of measures (3.5), and the relation between the sum-product theorem and growth in the affine group (\$3.3.1). In $\$ 3.3 .2$, we will take a brief look at solvable and nilpotent groups in general.

Arithmetic combinatorics is important for the history of the subject, and also provides part of its raison d'être; the study of growth in non-commutative groups, after all, could be said to be just the non-commutative case of arithmetic combinatorics. There is more to arithmetic combinatorics and to growth in groups than that, but there is still some truth to the statement, particularly in view of the genesis of the material we aim to describe.

3.1. Triple products and approximate subgroups. Some of additive combinatorics can be described as the study of sets that grow slowly. In abelian groups, results are often stated so as to classify sets $A$ such that $\left|A^{2}\right|$ is not much larger than $|A|$; in non-abelian groups, works, starting with Hel08, classify sets $A$ such that $\left|A^{3}\right|$ is not much larger than $|A|$.

There is a reason for this difference in conventions. In an abelian group, if $\left|A^{2}\right|<$ $K|A|$, then $\left|A^{k}\right|<K^{O(k)}|A|$-i.e., if a set does not grow after one multiplication with itself, it will not grow under many. This is a result of Plünnecke Plü70. and Ruzsa Ruz89. (Petridis Pet12 recently gave a purely additive-combinatorial proof.) In a non-abelian group $G$, there can be sets $A$ breaking this rule: for example, if $H \triangleleft G, g \in G \backslash H$ and $A=H \cup\{g\}$, then $\left|A^{2}\right|<3|A|$, but $A^{3} \supset H g H$, and $H g H$ can be much larger than $A$. (For instance, if $H$ is the subgroup of $G=\mathrm{SL}_{2}(\mathbb{Z} / p \mathbb{Z})$ leaving a basis vector $e_{1}$ fixed, and $w$ is the element of $G$ taking $e_{1}$ to $e_{2}$ and $e_{2}$ to $-e_{1}$, then $H w H$ is of size $|H|^{2}$. We will later see (proof of Proposition 5.2) that this is not an isolated example - it can be quite useful to stick a subgroup $H$ in different directions (so to speak) in order to get a large product.)

However, Ruzsa's ideas do carry over to the non-abelian case, as was pointed out in Hel08 and [Tao08; in fact RT85 carries over without change, since the assumption that $G$ is abelian is never really used. We must assume that $\left|A^{3}\right|$ is small, not just $\left|A^{2}\right|$, and then it does follow that $\left|A^{k}\right|$ is small.

Lemma 3.1 (Ruzsa triangle inequality). Let $A, B$, and $C$ be finite subsets of a group $G$. Then

$$
\left|A C^{-1}\right||B| \leq\left|A B^{-1}\right|\left|B C^{-1}\right|
$$

Commutativity is not needed. In fact, what is being used is in some sense more basic than a group structure; as shown in [GHR], the same argument works naturally in any abstract projective plane endowed with the little Desargues axiom.

Proof. We will construct an injection $\iota: A C^{-1} \times B \hookrightarrow A B^{-1} \times B C^{-1}$. For every $d \in A C^{-1}$, choose $\left(f_{1}(d), f_{2}(d)\right)=(a, c) \in A \times C$ such that $d=a c^{-1}$. Define $\iota(d, b)=\left(f_{1}(d) b^{-1}, b\left(f_{2}(d)\right)^{-1}\right)$. We can recover $d=f_{1}(d)\left(f_{2}(d)\right)^{-1}$ from $\iota(d, b)$; hence we can recover $\left(f_{1}, f_{2}\right)(d)=(a, c)$, and thus $b$ as well. Therefore, $\iota$ is an injection. 
It follows easily that

$$
\frac{\left|\left(A \cup A^{-1} \cup\{e\}\right)^{3}\right|}{|A|} \leq\left(3 \frac{\left|A^{3}\right|}{|A|}\right)^{3}
$$

for any finite subset $A$ of any group $G$, and, moreover,

$$
\frac{\left|A^{k}\right|}{|A|} \leq\left(\frac{\left|A^{3}\right|}{|A|}\right)^{k-2}
$$

for any $A \subset G$ such that $A=A^{-1}$ (i.e., $A$ contains the inverse of every element in $A$ ). (Both of these statements go back to Ruzsa (or Ruzsa and Turjányi [RT85]), at least for $G$ abelian.) Again, this is the sort of statement for which it is nicer to give a proof oneself than to read another person's proof. Let us do one case: $\left|A A^{-1} A\right||A|=\left|A A^{-1} A\right|\left|A^{-1}\right| \leq\left|A^{2} \| A^{-1} A^{-1} A\right|$ (by Lemma 3.1 with $B=A^{-1}$ and $C=A^{-1} A$ ) and $\left|A^{-1} A^{-1} A\right||A| \leq\left|A^{-1} A^{-1} A^{-1}\right|\left|A^{2}\right|=\left|A^{3}\right|\left|A^{2}\right|$ (again by Lemma 3.1), implying $\left|A A^{-1} A\right| /|A| \leq\left|A^{2}\right|^{2}\left|A^{3}\right| /|A|^{3} \leq\left(\left|A^{3}\right| /|A|\right)^{3}$. The rest of (3.2) and (3.3) is left as an exercise.

Because of (3.2) and (3.3), from now on we can generally focus on studying when $\left|A^{3}\right|$ is or is not much larger than $|A|$, assuming, without any essential loss of generality, that $A=A^{-1}$ and $e \in A$. Obviously, we can apply (3.3) to $A \cup A^{-1} \cup\{e\}$ after applying (3.2).

The paper Tao08 focused on translating several results from additive combinatorics to the non-abelian context. In the course of this task, Tao defined what he called an approximate group. (Approximate subgroup might be more suggestive, as will become clear in 4 .) A $K$-approximate subgroup of a group $G$ is a set $A \subset G$ such that

(a) $A=A^{-1}$ and $e \in A$,

(b) there is a subset $X \subset G$ such that $|X| \leq K$ and $A^{2} \subset X \cdot A$.

This is essentially equivalent to the notion of a slowly growing set $A$ (or a set of small tripling), that is a set for which $\left|A^{3}\right| \leq K^{\prime}|A|$ : a $K$-approximate group is a slowly growing set (trivially, with $K^{\prime}=K^{2}$ ) and, for a slowly growing set $A$ with $A=A^{-1}$ and $e \in A$, the set $A^{3}$ is a $K^{O(1)}$-approximate subgroup. This was shown by Tao [Tao08, Cor. 3.11], with the essential ingredient being the Ruzsa covering lemma ([Ruz99]).

Lemma 3.2 (Ruzsa covering lemma). Let $A$ and $B$ be finite subsets of a group $G$. Assume $|A \cdot B| \leq K|B|$. Then there is a subset $X \subset A$ with $|X| \leq K$ such that $A \subset X \cdot B \cdot B^{-1}$.

Proof. Let $\left\{a_{1}, a_{2}, \ldots, a_{k}\right\}$ be a maximal subset of $A$ with the property that the cosets $a_{j} B, 1 \leq j \leq k$, are all disjoint. It is clear that $k \leq|A \cdot B| /|B| \leq K$. Let $x \in A$. Since $\left\{a_{1}, a_{2}, \ldots, a_{k}\right\}$ is maximal, there is a $j$ such that $a_{j} B \cap x B$ is non-empty. Then $x \in a_{j} B B^{-1}$. Thus, the sets $a_{j} B B^{-1}$ cover $A$.

Tao also showed that one can classify sets $A$ of small doubling in terms of approximate subgroups, using the covering lemma as one of the main tools:

Lemma 3.3 ([Tao08, Cor. 4.7]). Let $A$ be a finite subset of a group $G$. If $|A \cdot A| \leq$ $K|A|$ or $\left|A \cdot A^{-1}\right| \leq K|A|$, then $A$ lies in the union of at most $O\left(K^{O(1)}\right)$ cosets of an $O\left(K^{O(1)}\right)$ approximate subgroup $H$ of size $|H| \ll K^{O(1)}|A|$. 
We will now see that the notion of sets of small growth, besides being essentially equivalent to that of approximate subgroups, are also closely connected to another growth-related concept, this one based on measures.

3.2. Balog-Szemerédi-Gowers theorem. Flattening lemma (Bourgain and Gamburd). The Balog-Szemerédi-Gowers theorem is a key tool in modern additive combinatorics. As we shall see, it is also important in the study of growth on groups, since it allows one to pass from results on sets to results on measures. The first version of the theorem was due to Balog and Szemerédi BS94. Gowers Gow98, Prop. 12] improved the bounds dramatically, making all dependencies polynomial; this is needed for our applications. Then Tao showed that the proof (which is essentially graph-theoretical, as shown in [SSV05]) also works in a non-commutative setting [Tao08, §5].

First, we need a definition. Its commutative counterpart, the additive energy, is very common in additive combinatorics.

Definition 1. Let $G$ be a group. Let $A, B \subset G$ be finite sets. The multiplicative energy $E(A, B)$ is

$E(A, B)=\sum_{g \in G}\left|\left(1_{A} * 1_{B}\right)(g)\right|^{2}=\left|\left\{\left(a_{1}, a_{2}, b_{1}, b_{2}\right) \in A \times A \times B \times B: a_{1} b_{1}=a_{2} b_{2}\right\}\right|$.

Here, as usual, the convolution $f * g$ is defined by

$$
(f * g)=\sum_{y} f(y) g\left(y^{-1} x\right) .
$$

Clearly, $E(A, B) \leq \min \left(|A|^{2}|B|,|A||B|^{2}\right)$. By the Cauchy-Schwarz inequality, we also have

$$
E(A, B) \cdot|A B| \geq\left|1_{A} * 1_{B}\right|_{1}^{2}=|A|^{2}|B|^{2} .
$$

In other words, if $|A B|$ is not much larger than $|A|$ or $|B|$, then $E(A, B)$ is large. It turns out that, while the naïve converse does not hold (in that, even if $E(A, B)$ is large, $|A B|$ can be much larger than $|A|$ and $|B|)$, a converse statement of sorts is true.

Proposition 3.4 (Non-commutative Balog-Szemerédi-Gowers theorem [Tao08]). Let $G$ be a group. Let $A, B \subset G$ be finite. Suppose that $E(A, B) \geq|A|^{3 / 2}|B|^{3 / 2} / K$. Then there are $A^{\prime} \subset A, B^{\prime} \subset B$ such that $\left|A^{\prime}\right| \gg|A| / K,\left|B^{\prime}\right| \gg|B| / K$ and $\left|A^{\prime} \cdot B^{\prime}\right| \ll K^{8} \sqrt{|A||B|}$, where the implied constants are absolute.

The Balog-Szemerédi-Gowers theorem (for $G$ commutative) already played a minor role in [Hel08; BG08b] would later show this was not necessary. What concerns us most here is its use for $G$ non-commutative in BG08c: Bourgain and Gamburd showed how to use Proposition 3.4 to reduce a statement on the "flattening" of measures to a statement about the growth of sets (namely, Theorem 1.1).

Again, this will be some sort of converse to an easier statement. The easier statement here would be that, if $A$ is a set satisfying $\left|A^{2}\right| \leq K|A|$, then $|\mu * \mu|_{2} \geq K^{-1}|\mu|_{2}$ for $\mu=(1 /|A|) \mu_{A}$. That is an exercise. (Hint: Write $\left|\mu_{A} * \mu_{A}\right|_{2}^{2}=E(A, A) /|A|^{4}$ and use (3.4) to bound $E(A, A)$.)

Proposition 3.5 ([BG08c], "Flattening lemma"). Let $G$ be a finite group. Let $\mu$ be a probability measure on $G$ with $\mu(g)=\mu\left(g^{-1}\right)$ for all $g \in G$. Suppose that

$$
|\mu * \mu|_{2} \geq K^{-1}|\mu|_{2}
$$


for some $K>0$. Then there is a $K^{O(1)}$-approximate subgroup $H \subset G$ of size $\ll K^{O(1)} /|\mu|_{2}^{2}$ and an element $g \in G$ such that $\mu(H g) \gg K^{-O(1)}$. (The implied constants are absolute.)

Note that $\mu(H g) \gg K^{-O(1)}$ implies $(\mu * \mu)\left(H^{2}\right) \geq \mu(H g) \mu\left(g^{-1} H\right) \gg K^{-O(1)}$ (since $\mu(g)=\mu\left(g^{-1}\right)$ and $\left.H=H^{-1}\right)$.

We will give Bourgain and Gamburd's proof with a technical simplification due to Tao Tao. Wigderson seems to have suggested an analogous simplification (based on an idea already in BIW06]).

Proof. Just for expository purposes, we consider first the case of $\mu=(1 /|A|) 1_{A}$, where $1_{A}$ is the characteristic function of a set $A \subset G$ (i.e., $1_{A}(g)=1$ if $g \in A$, $1_{A}(g)=0$ if $\left.g \notin A\right)$. Then

$$
|\mu * \mu|_{2}^{2}=\frac{1}{|A|^{4}} E(A, A), \quad|\mu|_{2}^{2}=\frac{1}{|A|} .
$$

Thus, (3.5) means that $E(A, A) \geq K^{-2}|A|^{3}$. Hence, by Proposition 3.4, there are $A_{1}^{\prime}, A_{2}^{\prime} \subset A$ such that $\left|A_{1}^{\prime}\right|,\left|A_{2}^{\prime}\right| \gg|A| / K^{2}$ and $\left|A_{1}^{\prime} A_{2}^{\prime}\right| \ll K^{18} \sqrt{\left|A_{1}^{\prime}\right|\left|A_{2}^{\prime}\right|}$. By the Ruzsa triangle inequality (3.1), $\left|A_{1}^{\prime} A_{1}^{\prime}\right| \ll K^{36}\left|A_{1}^{\prime}\right|$. Thus, by Lemma 3.3. $A_{1}^{\prime}$ lies in a union of $\ll K^{O(1)}$ cosets of an $O\left(K^{O(1)}\right)$ approximate subgroup $H$ of size $\ll K^{O(1)}\left|A_{1}^{\prime}\right| \leq K^{O(1)}|A|$. At least one of these cosets $H g$ must contain $\gg K^{-O(1)}\left|A_{1}^{\prime}\right|$ elements of $A_{1}^{\prime}$, and thus of $A$. Hence $\mu(H g) \gg K^{-O(1)}$.

Now consider the case of general $\mu$. The idea is that (thanks in part to (3.5)) the bulk of $\mu$ is given by the values $\mu(g)$, neither much larger nor much smaller than a certain value $a$; that "bulk" (call it $\mu_{\sim}$ ) behaves essentially as a characteristic function, thus reducing the situation to the one we have already considered.

Inspired by the second equation in (3.6), we define $a=|\mu|_{2}^{2}$, and let $A$ be the set of all $g \in G$ with $\mu(g) \geq a /\left(C K^{c}\right)$, where $c, C>0$ will be set later. We let $\mu_{A}=(1 /|A|) 1_{A}$; we must check that $\left|\mu_{A} * \mu_{A}\right|_{2}$ is large relative to $\left|\mu_{A}\right|_{2}=1 / \sqrt{|A|}$.

First, note that each $g \in A$ makes, by definition, a contribution of $\geq a^{2} /\left(C^{2} K^{2 c}\right)$ to $|\mu|_{2}^{2}$; hence $|A| \leq C^{2} K^{2 c} / a$, and so $1 /|A| \geq a /\left(C^{2} K^{2 c}\right)$.

We split $\mu=\mu_{<}+\mu_{\sim}+\mu_{>}$, where $\mu_{<}(g)=\mu(g)$ when $\mu(g)<a /\left(C K^{c}\right)$ and 0 otherwise, and $\mu_{>}(g)=\mu(g)$ when $\mu(g)>C K^{c} a$ and 0 otherwise. Now $|f * g|_{2} \leq$ $|f|_{2}|g|_{1}$ for any $f, g$ (Young's inequality, special case; follows from the CauchySchwarz inequality). Hence,

$$
\begin{aligned}
& \left|\mu * \mu_{<}\right|_{2}=\left|\mu_{<} * \mu\right|_{2} \leq|\mu|_{1}\left|\mu_{<}\right|_{2} \leq 1 \cdot \sqrt{\left|\mu_{<}\right|_{\infty}\left|\mu_{<}\right|_{1}} \leq \frac{\sqrt{a}}{\sqrt{C K^{c}}} \\
& \left|\mu_{>} * \mu\right|_{2}=\left|\mu * \mu_{>}\right|_{2} \leq\left|\mu_{>}\right|_{1}|\mu|_{2} \leq \frac{\left|\mu_{>}\right|_{2}^{2}}{\min _{g \in G}\left|\mu_{>}(g)\right|} \sqrt{a} \leq \frac{a^{3 / 2}}{C K^{c} a} \leq \frac{\sqrt{a}}{\sqrt{C K^{c}}} .
\end{aligned}
$$

Thus, we can afford to cut off the tails: we obtain, by (3.5),

$$
\left|\mu_{\sim} * \mu_{\sim}\right|_{2} \geq K^{-1} \sqrt{a}-\frac{4 \sqrt{a}}{\sqrt{C K^{c}}} \geq \frac{1}{5} K^{-1} \sqrt{a},
$$

where we have set $C=5, c=2$. We are almost done; we now need to go from $\mu_{\sim}$, which is roughly a characteristic function, to $\mu_{A}$, which is actually a characteristic function.

The inequality (3.7) enables us to bound

$$
\left|\mu_{A} * \mu_{A}\right|_{2} \geq \frac{1 /|A|}{\left|\mu_{\sim}\right|_{\infty}}\left|\mu_{\sim} * \mu_{\sim}\right|_{2} \geq \frac{1 /|A|}{C K^{c} a} \cdot \frac{1}{5} K^{-1} \sqrt{a}=\frac{1}{25 K^{3}} \frac{1 /|A|}{\sqrt{a}} .
$$


By $1 /|A| \geq a /\left(C^{2} K^{2 c}\right)=a /\left(5^{2} K^{4}\right)$ and $\left|\mu_{A} * \mu_{A}\right|_{2}^{2}=E(A, A) /|A|^{4} \leq 1 /|A|$, this implies both

$$
\left|\mu_{A} * \mu_{A}\right|_{2} \geq \frac{1 /|\sqrt{A}|}{5^{3} K^{5}}=\frac{\left|\mu_{A}\right|_{2}}{5 K^{2}} \quad \text { and } \quad|A| \geq \frac{1 / a}{5^{4} K^{6}} .
$$

We now have the setup we had at the beginning, only with $\mu_{A}$ instead of $\mu$ and $5^{3} K^{5}$ instead of $K$. Proceeding as before, we obtain a $K^{O(1)}$-approximate subgroup $H \subset K$ such that $\mu_{A}(H g) \gg K^{-O(1)}$ for some $g \in G$, and so

$$
\mu(H g) \geq \frac{a}{5 K^{2}} 1_{A}(H g)=\frac{a|A|}{5 K^{2}} \mu_{A}(H g) \gg K^{-O(1)} .
$$

\subsection{The sum-product theorem. Growth in solvable groups.}

3.3.1. The affine group and the sum-product theorem. The analogue of the following lemma over $\mathbb{R}$ had been known for a long while (Erdös and Szemerédi [ES83]). The version over finite fields is harder, since there is no natural topology or fully natural ordering to work with. (Over $\mathbb{R}$, there is a brief and very natural proof Ele97. based on a result that is essentially topological [ST83; the best-known bound for the sum-product theorem over $\mathbb{R}$ has a direct proof, also topological [Sol09].)

Theorem 3.6 (Sum-product theorem [BKT04], BGK06]; see also [EM03]). For any $A \subset \mathbb{F}_{p}^{*}$ with $C<|A|<p^{1-\epsilon}, \epsilon>0$, we have

$$
\max (|A \cdot A|,|A+A|)>|A|^{1+\delta},
$$

where $C>0$ and $\delta>0$ depend only on $\epsilon$.

The proof was strengthened and simplified in TV06] and GK07]

The same result holds for $\mathbb{F}_{q}, q=p^{a}$, and indeed for arbitrary fields; we must only be careful to specify that $A$ is not concentrated in a proper subfield. The strength of this result must be underlined: $A$ is growing by a factor of $|A|^{\delta}$, where $\delta>0$ is moreover independent of $p$. In contrast, even after impressive recent improvements ( San12], see also [CS10]), the main additive-combinatorial result for abelian groups (Freiman's theorem) gives growth by smaller factors.

Rather than prove Theorem 3.6 , let us prove the key intermediate result toward it; it is enough for many applications, and it also illustrates the connection between the sum-product theorem and growth in solvable groups. The following idea was put forward in [Hel11, §3.1] and developed there and in later works: the sum-product theorem is really a result about the action of a group on another group; in its usual formulation (Theorem 3.6), the group that is acting is $\mathbb{F}_{p}^{*}$ (by multiplication), and the group being acted upon is $\mathbb{F}_{p}^{+}$.

Let $G$ be the affine group

$$
G=\left\{\left(\begin{array}{cc}
r & a \\
0 & 1
\end{array}\right): r \in \mathbb{F}_{p}^{*}, a \in \mathbb{F}_{p}\right\}
$$

Consider the following subgroups of $G$ :

$$
U=\left\{\left(\begin{array}{cc}
1 & a \\
0 & 1
\end{array}\right): a \in \mathbb{F}_{p}\right\}, \quad T=\left\{\left(\begin{array}{cc}
r & 0 \\
0 & 1
\end{array}\right): r \in \mathbb{F}_{p}^{*}\right\} .
$$

These are simple examples of a solvable group $G$, of a maximal unipotent subgroup $U$ and of a maximal torus $T$. Actually, the centralizer $C(g)$ of any element $g$ of $G$ not in $\pm U$ is a maximal torus. 
We look at two actions - that of $U$ on itself (by the group operation) and that of $T$ on $U$ (by conjugation; $U$ is a normal subgroup of $G$ ). They turn out to correspond to addition and multiplication in $\mathbb{F}_{p}$, respectively:

$$
\begin{aligned}
\left(\begin{array}{cc}
1 & a_{1} \\
0 & 1
\end{array}\right) \cdot\left(\begin{array}{cc}
1 & a_{2} \\
0 & 1
\end{array}\right) & =\left(\begin{array}{cc}
1 & a_{1}+a_{2} \\
0 & 1
\end{array}\right), \\
\left(\begin{array}{ll}
r & 0 \\
0 & 1
\end{array}\right) \cdot\left(\begin{array}{ll}
1 & a \\
0 & 1
\end{array}\right) \cdot\left(\begin{array}{cc}
r^{-1} & 0 \\
0 & 1
\end{array}\right) & =\left(\begin{array}{cc}
1 & r a \\
0 & 1
\end{array}\right) .
\end{aligned}
$$

Thus, we see that growth in $U$ (under the actions of $U$ and $T$ ) is tightly linked to growth in $\mathbb{F}_{p}$ (under addition and multiplication).

In fact, the result we will prove on these two actions (Proposition 4.7), implies immediately the "key intermediate result" we want:

Proposition 3.7 (GK07, Corollary 3.5). Let $X \subset \mathbb{F}_{p}, Y \subset \mathbb{F}_{p}^{*}$ be given with $X=-X, 0 \in X, 1 \in Y$. Then

$$
\left|4 Y X+2 Y^{2} X\right| \geq \frac{1}{2} \min (|X||Y|, p) .
$$

We write $4 X$ (say) for $X^{4}$ when $X$ is a subset of an additive group; thus, e.g., $2 Y^{2} X=Y^{2} X+Y^{2} X$.

Theorem 3.6 follows from Proposition 3.7 after the application of the Katz-Tao lemma [TV06, §2.8], which plays a role for sums and products analogous to that played by Lemma 3.1 (Ruzsa) for group operations.

3.3.2. Solvable and nilpotent groups. Before we actually start working on the affine group $(\$ 4.2)$, let us take a look at the general situation for solvable and nilpotent groups. This will not be needed for our treatment of the affine group, but it will be a chance to consider several intriguing questions, some of them going outside our main framework.

N. Gill and the author $[\mathrm{GH}]$ proved growth in all solvable subgroups of $\mathrm{GL}_{n}\left(\mathbb{F}_{p}\right)$, in the sense of Proposition 4.8. The main two challenges were the existence of elements outside $U$ that are not semisimple (and thus their action on $U$ has nontrivial fixed points) and the relatively complicated subgroup structure that solvable subgroups of $\mathrm{GL}_{n}\left(\mathbb{F}_{p}\right)$ can have. The case of solvable groups over $\mathbb{F}_{q}$ remains open; a proof along the lines of $\mathrm{GH}$ ] should be feasible but cumbersome. (The case of $\mathrm{GL}_{n}\left(\mathbb{F}_{q}\right)$ does not reduce to $\mathrm{GL}_{n}\left(\mathbb{F}_{p}\right)$, since doing that in a naive way would increase the rank $n$ depending on $q$, and we want results independent on $p$ or $q$.) As usual in this context, infinite fields can be easier if they have a "sensible" topology and/or if the subgroup structure is simpler ([BG11a], BG11b, BG12]). The problem also becomes more accessible if, instead of aiming at bounds of the quality $\left|A^{3}\right| \geq|A|^{1+\delta}$, we aim at weaker bounds [Tao10], since then more tools are admissible.

Already in $G$ as in (3.8), growth-related behavior can be complex. We will show that subsets $A$ of $G$ do grow rapidly under the group operation, outside some very specific circumstances. However, the action of $G$ on $U$ does not, in general, give us expander graphs. To be precise: identify $U$ with $\mathbb{F}_{p}$, fix a $\lambda \in \mathbb{Z}^{+}$, and say we have $\epsilon$-expansion if, for every $S \subset \mathbb{F}_{p}$ with $|S|<p / 2$,

$$
|S \cup(S+1) \cup \lambda S| \geq(1+\epsilon)|S| .
$$

(Here the addition of 1 can be thought of as coming from the action of $U$ on itself, and multiplication by $\lambda$ comes from the action of $G / U$ on $U$ by conjugation.) 
In order to relate expansion in the sense of (3.10) to eigenvalues of graphs, it is necessary to define Schreier graphs. A Schreier graph is given by a set of actions $A$ on a set $X$ (here $X=\mathbb{F}_{p}$ ); the set of vertices is $X$, and the set of edges is $\{(x, a(x)): x \in X, a \in A\}$. (A Cayley graph is thus a special case of a Schreier graph: $A \subset X, A$ acts on $X$ by multiplication.) Now, the spectrum of the discrete Laplacian for the Schreier graph $\Gamma$ given by $x \mapsto x+1, x \rightarrow \lambda x$ is given in MV00, which, in particular, shows that there is no constant spectral gap, i.e., $\lambda_{1}$ is not bounded away from 0 as $p \rightarrow \infty$; this implies that there is no fixed $\epsilon>0$ such that (3.10) holds for all $S \subset \mathbb{F}_{p}$ with $|S| \geq p / 2$ and all sufficiently large $p$.

J. Cilleruelo points out that one can prove this directly by modifying a construction by G. Fiz Pontiveros [Fiz, based in turn on an idea of Rokhlin's [Rok63] let $I$ be the reduction of $\{0 \leq n \leq \epsilon p / 3\}$ modulo $p$, and let $\phi: \mathbb{Z} / p \mathbb{Z} \rightarrow \mathbb{Z} / p \mathbb{Z}$ be the multiplication-by- $\lambda$ map; and define

$$
S=\bigcup_{0 \leq i \leq \frac{2}{\epsilon}} \phi^{-1}(I) .
$$

Then $|S| \sim p / 3$ and $|S \cup(S+1) \cup \lambda S| \leq(1+\epsilon)|S|$ for any $p$ larger than a constant depending only on $\epsilon$.

Can one make the somewhat weaker assertion that the diameter of the Schreier graph $\Gamma$ is small? For $\lambda \in \mathbb{Z}^{+}$, this is very easy: writing $x$ in base $\lambda$, we obtain that the diameter is $O_{\lambda}(\log x)$. If we want a bound independent of $\lambda$ for $\lambda \in \mathbb{F}_{p}^{*}$ arbitrary, the problem is subtler. (We must impose the condition that the order of $\lambda$ not be too small, or else what we try to prove could be false.) Using a result of Konyagin [Kon92, it is possible to show that the diameter is $O\left((\log |G|)^{O(1)}\right)$, where both implied constants are absolute 4

Nilpotent groups. The case of nilpotent groups is fairly close to the special case of abelian groups. Here Fisher, Katz, and Peng [FKP10] and Tao [Tao10] laid the groundwork; the recent preprint [Toi] contains a general statement. In summarytools that yield Freiman's theorem over abelian groups (also called Freiman-Ruzsa in that generality) can be adapted to work over nilpotent groups. On the flip side, bounds are quantitatively weak and necessarily conditional on the non-existence of significant structure (progressions), just as in the abelian case.

Finally, let us take a brief look at the asymptotics of growth in infinite solvable and nilpotent groups. Here some of the main results are Wol68, Mil68, Bas72, Gui73; in summary, a set of generators $A$ of a solvable group $G$ has polynomial growth (i.e., $\left|A^{k}\right| \ll|A|^{O(1)}$ ) if and only if $G$ has a nilpotent subgroup of finite index. (When $G$ is not assumed to be solvable, the "only if" direction becomes very hard; this is due to Tits for linear groups (a consequence of the "Tits alternative" [Tit72]) and to Gromov for general groups (the celebrated paper [Gro81]).)

\section{Group aCtions: Stabilizers, orbits, AND PIVOTS}

4.1. The orbit-stabilizer theorem for sets. A leitmotif recurs in recent work on growth in groups: results on subgroups can often be generalized to subsets. This is especially the case if the proofs are quantitative, constructive, or, as we shall later see, probabilistic.

\footnotetext{
${ }^{4}$ This was the outcome of a discussion among B. Bukh, A. Harper, and the author. We thank E. Lindenstrauss for referring us to Konyagin's paper.
} 
The orbit-stabilizer theorem for sets is an example both paradigmatic and basic: It underlies a surprising number of other results on growth. It also helps to put forward a case for seeing group actions, rather than groups themselves, as the main object of study. We state it as in [HS14, §3.1], though it is already implicit in [Hel08] (and clear in [Hel11]).

We recall that an action $G \curvearrowright X$ is a homomorphism from a group $G$ to the group of automorphisms of a set $X$. (The automorphisms of a set $X$ are just the bijections from $X$ to $X$; we will see actions on objects with richer structures later.) For $A \subset G$ and $x \in X$, the orbit $A x$ is the set $A x=\{g \cdot x: g \in A\}$. The stabilizer $\operatorname{Stab}(x) \subset G$ is given by $\operatorname{Stab}(x)=\{g \in G: g \cdot x=x\}$.

(Permutation group theorists prefer to use actions on the right; they write $x^{g}$ for $g(x), G_{x}$ for $\operatorname{Stab}(x)$, and use right cosets by default. We will use that notation in $₫ 6$, where we will also write $x^{A}$ instead of $A x$, in consequence.)

Lemma 4.1 (Orbit-stabilizer theorem for sets). Let $G$ be a group acting on a set $X$. Let $x \in X$, and let $A \subseteq G$ be non-empty. Then

$$
\left|\left(A^{-1} A\right) \cap \operatorname{Stab}(x)\right| \geq \frac{|A|}{|A x|} .
$$

Moreover, for every $B \subseteq G$,

$$
|B A| \geq|A \cap \operatorname{Stab}(x)||B x| .
$$

The usual orbit-stabilizer theorem is the special case $A=B=H, H$ a subgroup of $G$.

Sketch of proof. Exercise: (4.1) is proven by pigeonhole, (4.2) by counting.

Let $H$ be a subgroup of $G$. The following lemmas are all direct consequences of the above for the natural action $G \curvearrowright X=G / H$ defined by group multiplication. Lemma 4.2 gives us elements in a subgroup of $G$; Lemmas 4.3 and 4.4 tell us that, to obtain growth in a group, it is enough to obtain growth in a subgroup or in a quotient.

Lemma 4.2 ([Hel11, Lem. 7.2]). Let $G$ be a group and $H$ a subgroup thereof. Let $A \subset G$ be a non-empty set. Then

$$
\left|A A^{-1} \cap H\right| \geq \frac{|A|}{r},
$$

where $r$ is the number of cosets of $H$ intersecting $A$.

Lemma 4.3 ([HS14, Lem. 3.5]). Let $G$ be a group and $H$ a subgroup thereof. Let $A \subset G$ be a non-empty set with $A=A^{-1}$. Then, for any $k>0$,

$$
\left|A^{k+1}\right| \geq \frac{\left|A^{k} \cap H\right|}{\left|A^{2} \cap H\right|}|A| \text {. }
$$

Lemma 4.4 ([Hel11, Lem. 7.4]). Let $G$ be a group and $H$ a subgroup thereof. Write $\pi_{G / H}: G \rightarrow G / H$ for the quotient map. Let $A \subseteq G$ be a non-empty set with $A=A^{-1}$. Then, for any $k>0$,

$$
\left|A^{k+2}\right| \geq \frac{\left|\pi_{G / H}\left(A^{k}\right)\right|}{\left|\pi_{G / H}(A)\right|}|A| .
$$


Hints for Lemmas 4.2 4.4. Let $G \curvearrowright X=G / H$ be the natural action by multiplication, and let $x \in X$ be the equivalence class of the identity (i.e., $H$ ). For Lemma 4.3, use first (4.1), then (4.2) (with $A^{k}$ instead of $A$ and $A$ instead of $B$ for (4.2)). For Lemma 4.4, use first (4.2) (with $A^{2}$ instead of $A$ and $A^{k}$ instead of $B$ ), then (4.1).

In the above, as is often the case, the assumption $A=A^{-1}$ is inessential but convenient from the point of view of notation. (Obviously, if $A$ is a set not fulfilling $A=A^{-1}$, we can apply the lemmas to $A \cup A^{-1}$ rather than to $A$.)

As far as the orbit-stabilizer theorem (Lemma 4.1) is concerned, the action of $G$ on itself by multiplication is dull-all stabilizers are trivial. However, the action of $G$ on itself by conjugation is rather interesting. Write $C_{H}(g)$ for the centralizer

$$
C_{H}(g)=\left\{h \in H: h g h^{-1}=g\right\}
$$

and $\mathrm{Cl}(g)$ for the conjugacy class

$$
\mathrm{Cl}(g)=\left\{h g h^{-1}: h \in G\right\} .
$$

We can write $C(g)$ as short for $C_{G}(g)$.

Lemma 4.5. Let $A \subset G$ be a non-empty set with $A=A^{-1}$. Then, for every $g \in A^{l}, l \geq 1$,

$$
\left|A^{2} \cap C(g)\right| \geq \frac{|A|}{\left|A^{l+2} \cap \mathrm{Cl}(g)\right|} .
$$

Proof. Let $G \curvearrowright G$ be the action of $G$ on itself by conjugation. Apply (4.1) with $x=g$; the orbit of $g$ under conjugation by $A$ is contained in $A^{l+2} \cap \mathrm{Cl}(g)$.

4.2. A pivoting argument: the affine group. We will now see how to obtain growth in the affine group (3.8). The main ideas in the proof of Proposition 4.7 below were extracted in Hel11 from the proof of the sum-product theorem in [GK07]. In [Hel11, §3] and then in [GH], a similar strategy was shown to work for more general solvable groups. The theme of pivoting will recur in $\$ 5.4$.

First, let us see how to construct many elements in $U$ and $T$ starting from $A$.

Lemma 4.6. Let $G$ be the affine group over $\mathbb{F}_{p}$ (3.8). Let $U$ be the maximal unipotent subgroup of $G$, and let $\pi: G \rightarrow G / U$ be the quotient map.

Let $A \subset G, A=A^{-1}$. Assume $A \not \subset U$, and let $x$ be an element of $A$ not in $U$. Then

$$
\left|A^{2} \cap U\right| \geq \frac{|A|}{|\pi(A)|}, \quad\left|A^{2} \cap T\right| \geq \frac{|A|}{\left|A^{5}\right|}|\pi(A)|
$$

for $T=C(x)$.

Recall $U$ is given by (3.9) . Since $x \notin U$, its centralizer $T=C(x)$ is a maximal torus.

Proof. By Lemma 4.2 $A_{u}:=A^{-1} A \cap U$ has at least $|A| /|\pi(A)|$ elements. Consider the action of $G$ on itself by conjugation. Then, by Lemma 4.1, $\left|A^{-1} A \cap \operatorname{Stab}(x)\right| \geq$ $|A| /|A(x)|$. (Here $A(x)$ is the orbit of $x$ under the action (by conjugation) of $A$.) 
We set $A_{t}:=\left(A^{-1} A\right) \cap \operatorname{Stab}(x) \subset T$. Clearly, $|A(x)|=\left|A(x) x^{-1}\right|$ and $(A x) x^{-1} \subset$ $A^{4} \cap U$, and so $|A(x)| \leq\left|A^{4} \cap U\right|$. At the same time, by (4.2) applied to the action $G \curvearrowright G / U$ by left multiplication, $\left|A^{5}\right|=\left|A^{4} A\right| \geq\left|A^{4} \cap U\right| \cdot|\pi(A)|$. Hence

$$
\left|A_{t}\right| \geq \frac{|A|}{\left|A^{4} \cap U\right|} \geq \frac{|A|}{\left|A^{5}\right|}|\pi(A)| \text {. }
$$

As per previous notation, $A_{t}^{2}=A_{t} \cdot A_{t}, A_{t}\left(A_{u}\right)=\left\{t_{1}\left(u_{1}\right): t_{1} \in A_{t}, u_{1} \in A_{u}\right\}$, and $t(u)=t u t^{-1}$ (that is, $T$ acts on $U$ by conjugation).

Proposition 4.7. Let $G$ be the affine group over $\mathbb{F}_{p}$, let $U$ be the maximal unipotent subgroup of $G$, and let $T$ be a maximal torus. Let $A_{u} \subset U, A_{t} \subset T$. Assume $A_{u}=A_{u}^{-1}, e \in A_{t}, A_{u}$, and $A_{u} \nsubseteq\{e\}$. Then

$$
\left|\left(A_{t}^{2}\left(A_{u}\right)\right)^{6}\right| \geq \frac{1}{2} \min \left(\left|A_{u}\right|\left|A_{t}\right|, p\right) .
$$

To emphasize that what we prove really does imply Theorem 3.7 as stated, let us make clear that we will actually prove that

$$
\left|A_{t}\left(A_{u}\right) A_{t}^{2}\left(A_{u}\right) A_{t}\left(A_{u}^{2}\right) A_{t}^{2}\left(A_{u}\right) A_{t}\left(A_{u}\right)\right| \geq \frac{1}{2} \min \left(\left|A_{u}\right|\left|A_{t}\right|, p\right),
$$

which is slightly stronger than (4.6).

Proof. Call $a \in U$ a pivot if the function $\phi_{a}: A_{u} \times A_{t} \rightarrow U$ given by

$$
(u, t) \mapsto u t(a)=u t a t^{-1}
$$

is injective.

Case (a): There is a pivot a in $A_{u}$. Then $\left|\phi_{a}\left(A_{u}, A_{t}\right)\right|=\left|A_{u}\right|\left|A_{t}\right|$, and so

$$
\left|A_{u} A_{t}(a)\right| \geq\left|\phi_{a}\left(A_{u}, A_{t}\right)\right|=\left|A_{u}\right|\left|A_{t}\right| \text {. }
$$

This is the motivation for the name "pivot": the element $a$ is the pivot on which we build an injection $\phi_{a}$, giving us the growth we want.

Case (b): There are no pivots in $U$. As we are about to see, this case can arise only if either $A_{u}$ or $A_{t}$ is large with respect to $p$. Say that $\left(u_{1}, t_{1}\right),\left(u_{2}, t_{2}\right)$ collide for $a \in U$ if $\phi_{a}\left(u_{1}, t_{1}\right)=\phi_{a}\left(u_{2}, t_{2}\right)$. Saying that there are no pivots in $U$ is the same as saying that, for every $a \in U$, there are at least two distinct $\left(u_{1}, t_{1}\right),\left(u_{2}, t_{2}\right)$ that collide for $a$. Now, two distinct $\left(u_{1}, t_{1}\right),\left(u_{2}, t_{2}\right)$ can collide for at most one $a \in U \backslash\{e\}$. (The reader can quickly check this; the reason is that a collision corresponds to a solution to a non-trivial linear equation, which can have at most one solution.) Hence, if there are no pivots, $\left|A_{u}\right|^{2}\left|A_{t}\right|^{2} \geq|U \backslash\{e\}|=p-1$, i.e., $\left|A_{u}\right| \cdot\left|A_{t}\right|$ is large with respect to $p$. This already hints that this case will not be hard; it will yield to Cauchy-Schwarz and the like.

Choose the most "pivot-like" $a \in U$, meaning an element $a \in U$ such that the number of collisions

$$
\kappa_{a}=\left|\left\{u_{1}, u_{2} \in A_{u}, t_{1}, t_{2} \in A_{t}: \phi_{a}\left(u_{1}, t_{1}\right)=\phi_{a}\left(u_{2}, t_{2}\right)\right\}\right|
$$

is minimal. As we were saying, two distinct $\left(u_{1}, t_{1}\right),\left(u_{2}, t_{2}\right)$ collide for at most one $a \in U \backslash e$. Hence, the total number of collisions $\sum_{a \in U \backslash\{e\}} \kappa_{a}$ is $\leq\left|A_{u} \| A_{t}\right|(p-1)+$ $\left|A_{u}\right|^{2}\left|A_{t}\right|^{2}$, and so

$$
\kappa_{a} \leq \frac{\left|A_{u}\right|\left|A_{t}\right|(p-1)+\left|A_{u}\right|^{2}\left|A_{t}\right|^{2}}{p-1} \leq\left|A_{u}\right|\left|A_{t}\right|+\frac{\left|A_{u}\right|^{2}\left|A_{t}\right|^{2}}{p} .
$$


Cauchy-Schwarz implies that $\left|\phi_{a}\left(A_{u}, A_{t}\right)\right| \geq\left|A_{u}\right|^{2}\left|A_{t}\right|^{2} / \kappa_{a}$, and so

$$
\left|\phi_{a}\left(A_{u}, A_{t}\right)\right| \geq \frac{\left|A_{u}\right|^{2}\left|A_{t}\right|^{2}}{\left|A_{u}\right|\left|A_{t}\right|+\frac{\left|A_{u}\right|^{2}\left|A_{t}\right|^{2}}{p}}=\frac{1}{\frac{1}{\left|A_{u}\right|\left|A_{t}\right|}+\frac{1}{p}} \geq \frac{1}{2} \min \left(\left|A_{u}\right|\left|A_{t}\right|, p\right) .
$$

We are not quite done, since $a$ may not be in $A$. Since $a$ is not a pivot (as there are none), there exist distinct $\left(u_{1}, t_{1}\right),\left(u_{2}, t_{2}\right)$ such that $\phi_{a}\left(u_{1}, t_{1}\right)=\phi_{a}\left(u_{2}, t_{2}\right)$. Then $t_{1} \neq t_{2}$ (why?), and so the map $\psi_{t_{1}, t_{2}}: U \rightarrow U$ given by $u \mapsto t_{1}(u)\left(t_{2}(u)\right)^{-1}$ is injective. What follows may be thought of as the "unfolding" step, in that there is an element $a$ we want to remove from an expression, and we do so by applying to the expression a map that will send $a$ to something known. (We will be using the commutativity of $T$ here.)

For any $u \in U, t \in T$, since $T$ is abelian,

$$
\begin{aligned}
\psi_{t_{1}, t_{2}}\left(\phi_{a}(u, t)\right) & =t_{1}(u t(a))\left(t_{2}(u t(a))\right)^{-1}=t_{1}(u) t\left(t_{1}(a)\left(t_{2}(a)\right)^{-1}\right)\left(t_{2}(u)\right)^{-1} \\
& =t_{1}(u) t\left(\psi_{t_{1}, t_{2}}(a)\right)\left(t_{2}(u)\right)^{-1}=t_{1}(u) t\left(u_{1}^{-1} u_{2}\right)\left(t_{2}(u)\right)^{-1} .
\end{aligned}
$$

(Note that $a$ has just disappeared.) Hence,

$$
\psi_{t_{1}, t_{2}}\left(\phi_{a}\left(A_{u}, A_{t}\right)\right) \subset A_{t}\left(A_{u}\right) A_{t}\left(A_{u}^{2}\right) A_{t}\left(A_{u}\right) \subset\left(A_{t}\left(A_{u}\right)\right)^{4} .
$$

Since $\psi_{t_{1}, t_{2}}$ is injective, we conclude that

$$
\left|\left(A_{t}\left(A_{u}\right)\right)^{4}\right| \geq\left|\psi_{t_{1}, t_{2}}\left(\phi_{a}\left(A_{u}, A_{t}\right)\right)\right|=\left|\phi_{a}\left(A_{u}, A_{t}\right)\right| \geq \frac{1}{2} \min \left(\left|A_{u}\right|\left|A_{t}\right|, p\right) .
$$

There is an idea here that we are about to see again: any element $a$ that is not a pivot can, by this very fact, be given in terms of some $u_{1}, u_{2} \in A_{u}, t_{1}, t_{2} \in A_{t}$, and so an expression involving $a$ can often be transformed into one involving only elements of $A_{u}$ and $A_{t}$.

Case (c): There are pivots and non-pivots in $U$. This is what we can think of as an inductive step - essentially the same inductive step as in "escape from subvarieties" (to be discussed in \$5.1). To do induction, we do not really need an ordering; generation will suffice. Since $A_{u} \nsubseteq\left\{\{e\}, A_{u}\right.$ generates $U$. This implies that there is a non-pivot $a \in U$ and a $g \in A_{u}$ such that $g a$ is a pivot. Then $\phi_{a g}: A_{u} \times A_{t} \rightarrow U$ is injective. Much as in (4.7), we unfold:

$$
\begin{aligned}
\psi_{t_{1}, t_{2}}\left(\phi_{g a}(u, t)\right) & =t_{1}(u t(g) t(a))\left(t_{2}(u t(g) t(a))\right)^{-1} \\
& =t_{1}(u t(g)) t\left(u_{1}^{-1} u_{2}\right)\left(t_{2}(u t(g))\right)^{-1},
\end{aligned}
$$

where $\left(u_{1}, t_{1}\right),\left(u_{2}, t_{2}\right)$ are distinct pairs such that $\phi_{a}\left(u_{1}, t_{1}\right)=\phi_{a}\left(u_{2}, t_{2}\right)$. Just as before, $\psi_{t_{1}, t_{2}}$ is injective. Hence

$$
\left|A_{t}\left(A_{u}\right) A_{t}^{2}\left(A_{u}\right) A_{t}\left(A_{u}^{2}\right) A_{t}^{2}\left(A_{u}\right) A_{t}\left(A_{u}\right)\right| \geq\left|\psi_{t_{1}, t_{2}}\left(\phi_{g a}(u, t)\right)\right|=\left|A_{u}\right|\left|A_{t}\right| .
$$

The idea to recall here is that, if $S$ is a subset of an orbit $\mathscr{O}=\langle A\rangle x$ such that $S \neq \emptyset$ and $S \neq \mathscr{O}$, then there is an $s \in S$ and a $g \in A$ such that $g s \notin S$. In other words, we use the point at which we escape from $S$.

We are using the fact that $G$ is the affine group over $\mathbb{F}_{p}$ (and not over some other field) only at the beginning of Case (c) when we say that, for $A_{u} \subset U, A_{u} \nsubseteq\{e\}$ implies $\left\langle A_{u}\right\rangle=U$. 
Proposition 4.8. Let $G$ be the affine group over $\mathbb{F}_{p}$. Let $U$ be the maximal unipotent subgroup of $G$, and let $\pi: G \rightarrow G / U$ be the quotient map.

Let $A \subset G, A=A^{-1}, e \in A$. Assume $A$ is not contained in any maximal torus. Then either

$$
\left|A^{57}\right| \geq \frac{1}{2} \sqrt{|\pi(A)|} \cdot|A|
$$

or

$$
\left|A^{57}\right| \geq \frac{1}{2}|\pi(A)| p \quad \text { and } \quad U \subset A^{112} .
$$

Proof. We can assume $A \not \subset \pm U$, as otherwise what we are trying to prove is trivial. Let $g$ be an element of $A$ not in $\pm U$; its centralizer $C(g)$ is a maximal torus $T$. By assumption, there is an element $h$ of $A$ not in $T$. Then $h g h^{-1} g^{-1} \neq e$. At the same time, it does lie in $A^{4} \cap U$, and so $A^{4} \cap U$ is not $\{e\}$.

Let $A_{u}=A^{4} \cap U, A_{t}=A^{2} \cap T$; their size is bounded from below by (4.5). Applying Proposition 4.7, we obtain

$$
\left|A^{56} \cap U\right| \geq \frac{1}{2} \min \left(\left|A_{u}\right|\left|A_{t}\right|, p\right) \geq \frac{1}{2} \min \left(\frac{|A|}{\left|A^{5}\right|} \cdot|A|, p\right) .
$$

By (4.2), $\left|A^{57}\right| \geq\left|A^{56} \cap U\right| \cdot|\pi(A)|$. Clearly, if $|A| /\left|A^{5}\right|<1 / \sqrt{|\pi(A)|}$, then $\left|A^{57}\right| \geq$ $\left|A^{5}\right|>\sqrt{|\pi(A)|} \cdot|A|$.

The exponent 57 in (4.9) is not optimal, but, qualitatively speaking, Proposition 4.8 is as good a result as one can aim to for now: the assumption $A \not \subset T$ is necessary, the bound $\gg|\pi(A)| \cdot p$ can be tight when $U \subset A$. For $A \subset U$, getting a better-thantrivial bound amounts to Freiman's theorem in $\mathbb{F}_{p}$, and getting a growth factor of a power $|A|^{\delta}$ (rather than $\sqrt{|\pi(A)|}$ ) would involve getting a version of Freiman's theorem of polynomial strength (a difficult open problem).

Incidentally, (4.9) can be seen as a very simple result of the "classification of approximate subgroups" kind: if a set $A$ grows slowly $\left(\left|A^{k}\right| \leq|A|^{1+\delta}, k=57, \delta\right.$ small), then either $A$ is contained in a subgroup (a maximal torus) or $A$ is almost contained in a subgroup ( $U$, with "almost contained" meaning that $|\pi(A)| \leq|A|^{\delta}$ ) or $A^{k}$ contains a subgroup $(H=U)$ such that $\langle A\rangle / H$ is nilpotent (here, in fact, abelian).

A result of this kind was what $[\mathrm{GH}]$ proved for solvable subgroups and what Hel11 proved for $\mathrm{SL}_{3}\left(\mathbb{F}_{p}\right)$; that is to say, one can try to classify growth in general linear algebraic groups, leaving only the nilpotent case aside. This was called the "Helfgott-Lindenstrauss conjecture" in BGT12, which proved it in an impressively general but quantitatively very weak sense. In particular, BGT12 does recover a proof of Gromov's theorem (close to Hrushovski's [Hru12]), but it does not seem strong enough to give useful bounds for finite groups.

The use of pivoting for general groups was first advocated in [Hel11], but it came to full fruition only later, thanks to BGT11 and PSa: due in part to what in retrospect was a technical difficulty-namely, an intermediate result had an unnecessarily weak quantifier (see the remarks at the end of \$5.2) - Hel11 still uses a sum-product theorem at a certain point, though it does develop a more abstract setting, which we have just demonstrated here in the simplest case. 
Excursus. Let us comment briefly on how the sum-product theorem was used in the original proof of Theorem 1.1. The point is not (as one might naively think) that multiplying matrices involves summing products of matrix entries; that leads nowhere. Rather, the first proof of Theorem 1.1 used at one point the identity

$$
\operatorname{tr}(g) \operatorname{tr}(h)=\operatorname{tr}(g h)+\operatorname{tr}\left(g h^{-1}\right),
$$

valid for $\mathrm{SL}_{2}$, to show that, since the set $\operatorname{tr}(A)$ grows either under multiplication or addition (by the sum-product theorem), it grows both under multiplication and addition. Growth under addition then gets used to show that $\operatorname{tr}(A)$ grows under linear combinations, which are then achieved by multiplying elements of $A$ by a fixed element of $A$; thus, $\operatorname{tr}\left(A^{k}\right)$ grows as $k$ increases, and this is used to show that $A^{k}$ grows.

Later generalizations ( Hel11, GH11) showed that one can work with just about any linear identities involving traces instead of (4.11), provided that there are enough (independent) identities. This worked up to a certain point for $\mathrm{SL}_{n}$ and $\mathrm{Sp}_{2 n}$, but not for $\mathrm{SO}_{n}$, thereby frustrating a full proof for $\mathrm{SL}_{n}$. Both [BGT11] and PSa] showed one can do without such identities altogether.

\section{Growth in Linear Algebraic Groups}

Here we will go over an essentially complete and self-contained proof of Theorem 1.1. The proof we will give is somewhat more direct and easier to generalize than that in Hel08; it is influenced by Hel11, BGT11, PSa, and also by the exposition in Kow13. The basic elements are, however, the same: a dimensional estimate gives us tori with many elements on them, and, aided by an escape lemma, we will be able to use these tori to prove the theorem by contradiction, using a pivoting argument (indirectly in Hel08, directly here). The proof of the case of $\mathrm{SL}_{2}$ will be used to anchor a more general discussion; we will introduce the concepts used in the general case, explaining them by means of $\mathrm{SL}_{2}$. We will actually prove Theorem 1.1 for a general finite field $\mathbb{F}_{q}$, since we have no longer any use for the assumption that $q$ be prime.

We will then show how Theorem 1.1 and Proposition 3.5 imply Theorem 1.3 (Bourgain-Gamburd).

5.1. Escape. At some points in the argument, we will need to make sure that we can find an element $g \in A^{k}$ that is not special: for example, we want to be able to use a $g$ that is not unipotent, that does not have a given $\vec{v}$ as an eigenvector, that is regular semisimple (i.e., has a full set of distinct eigenvalues), etc. As [BGT11] states, arguments allowing us to do this appear in several places in the literature. The first version of Hel08 did this "by hand" in each case, and so does Kow13; that approach is useful if one aims at optimizing bounds, but our aim here is to proceed conceptually. The following general statement, used in Hel11, is modelled very closely after [EMO05, Prop. 3.2].

Lemma 5.1 (Escape). Let $G$ be a group acting linearly on a vector space $V / K$, and let $K$ be a field. Let $W$ be a subvariety of $V$ all of whose components have positive codimension in $V$. Let $A \subset G, A=A^{-1}, e \in A$, and let $x \in V$ be such that the orbit $\langle A\rangle \cdot x$ of $x$ is not contained in $W$.

Then there are constants $k, c$ depending only the number, dimension, and degree of the irreducible components of $W$ such that there are at least $\max (1, c|A|)$ elements $g \in A^{k}$ for which $g x \notin W$. 
In other words, if $x$ can escape from $W$ at all, it can escape from $W$ in a bounded number of steps.

Proof for a special case. Let us first prove the statement in the special case where $W$ is an irreducible linear subvariety. We will proceed by induction on the dimension of $W$. If $\operatorname{dim}(W)=0$, then $W$ consists of a single point, and the statement is clear: $\langle A\rangle \cdot x \not \subset\{x\}$ implies that there is a $g_{0} \in A$ such that $g_{0} x \neq x$; if there are fewer than $|A| / 2$ such elements of $A$, any product $g^{-1} g_{0}$ with $g x=x$ satisfies $g^{-1} g_{0} x \neq x$, and there are $>|A| / 2$ such products.

Assume, then, that $\operatorname{dim}(W)>0$, and that the statement has been proven for all $W^{\prime}$ with $\operatorname{dim}\left(W^{\prime}\right)<\operatorname{dim}(W)$. If $g W=W$ for all $g \in A$, then either (a) $g x$ does not lie on $W$ for any $g \in A$, proving the statement, or (b) $g x$ lies on $W$ for every $g \in\langle A\rangle$, contradicting the assumption. Assume that $g W \neq W$ for some $g \in A$; then $W^{\prime}=g W \cap W$ is an irreducible linear variety with $\operatorname{dim}\left(W^{\prime}\right)<\operatorname{dim}(W)$. Thus, by the inductive hypothesis, there are at least $\max \left(1, c^{\prime}|A|\right)$ elements $g^{\prime} \in A^{k^{\prime}}\left(c^{\prime}, k^{\prime}\right.$ depending only on $\left.\operatorname{dim}\left(W^{\prime}\right)\right)$ such that $g^{\prime} x$ does not lie on $W^{\prime}=g W \cap W$. Hence, for each such $g^{\prime}$, either $g^{-1} g^{\prime} x$ or $g^{\prime} x$ does not lie on $W$. We have thus proven the statement with $c=c^{\prime} / 2, k=k^{\prime}+1$.

Adapting the proof to the general case. Remove first the assumption of irreducibility; then $W$ is the union of $r$ components, not necessarily all of the same dimension. The intersection $W^{\prime}=g W \cap W$ may also have several components, but no more than $r^{2}$. Let $W_{1}$ be a component of $W$ of maximal dimension $d$. By the argument in the first sketch, we can find a $g \in A$ such that $g W_{1} \neq W_{1}$. (If $g x$ does not lie on $W_{1}$ for any $g \in A$, we simply remove $W_{1}$ from $W$ and repeat.) Hence $W^{\prime}=g W \cap W$ has fewer components of dimension $d$ than $W$ does. We can thus carry out the induction on (a) the maximum of the dimensions of the components of $W$, and (b) the number of components of maximal dimension. When (a) does not go down, it stays the same and (b) goes down; moreover, the number of components of lower dimension stays under control, as the total number of components $r$ gets no more than squared, as we said.

Removing the assumption that $V$ is linear is actually easy: the same argument works, and we only need to make sure that the total number of components (and their degree) stays under control. This is so by Bezout's theorem (in a general form, such as that in [DS98, p. 251], where the statement is credited to Fulton and MacPherson).

As Pyber and Szabó showed in [PSa], one can merge the "escape" argument above with the "dimensional estimates" we are about to discuss, in so far as that, in our context, an escape statement such as Lemma 5.1] is really a weak version of a dimensional estimate. Lemma 5.1tells us that many images $g x$ escape from a proper subvariety $W$, whereas a dimensional estimate tells us that, if $A$ grows slowly, very few images $g x, g \in A^{k}$, lie on a proper subvariety $W \subset G$. We will, however, use Lemma 5.1 as a tool to prove dimensional estimates and other statements, much as in [Hel11] (or BGT11]).

5.2. Dimensional estimates. By a dimensional estimate we mean a lower or upper bound on an intersection of the form $A^{k} \cap W$, where $A \subset G(K), W$ is a subvariety of $G$, and $G / K$ is an algebraic group. As the reader will notice, the 
bounds that we obtain will be meaningful when $A$ grows relatively slowly. However, no assumption on $A$ is made, other than that it generatt $5(K)$.

Let us first look at a particularly simple example. We will not actually use it as such here, but it was important in Hel08, and Hel11, and it exemplifies what is meant by a "dimensional estimate" and one way in which it can be proven. (Moreover, its higher-rank analogues do come into generalizations of what we will do to $\mathrm{SL}_{n}$ and other higher-rank groups, and the ideas in its proof will be reused for Proposition [5.3)

Proposition 5.2 ([Hel08, Lem. 4.7]; Hel11, Cor. 5.4], case $n=2$ ). Let $G=\mathrm{SL}_{2}$, and let $K$ be a field. Let $A \subset G(K)$ be a finite set with $A=A^{-1}, e \in A,\langle A\rangle=$ $G(K)$. Let $T$ be a maximal torus of $G$. Then

$$
|A \cap T(K)| \ll\left|A^{k}\right|^{1 / 3},
$$

where $k$ and the implied constant are absolute.

A maximal torus, in $\mathrm{SL}_{2}\left(\right.$ or $\mathrm{SL}_{n}$ ), is just the group of matrices that are diagonal with respect to some fixed basis of $\bar{K}^{2}$. Here $G(K)$ simply means the "set of $K$ valued points" of $G$, i.e., the group $\mathrm{SL}_{2}(K)$. (In general, according to standard formalism, an algebraic group is an abstract object (a variety plus morphisms); its set of $K$-valued points is a group.)

The meaning of $1 / 3$ in (5.1) is that it equals $\operatorname{dim}(T) / \operatorname{dim}(G)$. This will come through in the proof: we will manage to fit three copies of $T$ inside $G$ in, so to speak, independent directions.

Proof, as in [Hel11. It would be enough to construct an injective map

$$
\phi: T(K) \times T(K) \times T(K) \rightarrow G(K)
$$

such that $\phi(T(K) \cap A, T(K) \cap A, T(K) \cap A) \subset A^{k}$, since then

$$
\begin{aligned}
|T(K) \cap A|^{3} & =|(T(K) \cap A) \times(T(K) \cap A) \times(T(K) \cap A)| \\
& =|\phi((T(K) \cap A) \times(T(K) \cap A) \times(T(K) \cap A))| \leq\left|A^{k}\right| .
\end{aligned}
$$

It is easy to see that we can relax the condition that $\phi$ be injective; for example, it is enough to assume that every preimage $\phi^{-1}(g)$ has bounded size, and even then we can relax the condition still further by requiring only that $\phi^{-1}(g) \cap(T(K) \backslash S)^{3}$ be of bounded size, where $|S|$ is itself bounded, etc. Let us first construct $\phi$ and then see how far we have to relax injectivity.

We define $\phi: T(K) \times T(K) \times T(K) \rightarrow G(K)$ by

$$
\phi\left(t_{0}, t_{1}, t_{2}\right)=t_{0} \cdot g_{1} t_{1} g_{1}^{-1} \cdot g_{2} t_{2} g_{2}^{-1},
$$

where $g_{1}, g_{2} \in A^{k^{\prime}}, k^{\prime}=O(1)$, are about to be specified. It is easy to show that there are $g_{1}^{\prime}, g_{2}^{\prime} \in G(\bar{K})$ such that $v, g_{1}^{\prime} v g_{1}^{\prime-1}$ and $g_{2}^{\prime} v g_{2}^{\prime-1}$ are linearly independent, where $v$ is a non-zero tangent vector to $T$ at the origin. Now, the pairs $\left(g_{1}^{\prime}, g_{2}^{\prime}\right) \in$ $G(\bar{K}) \times G(\bar{K})$ for which $v, g_{1}^{\prime} v g_{1}^{\prime-1}, g_{2}^{\prime} v g_{2}^{\prime-1}$ are not linearly independent form a subvariety $W$ of $G \times G$ of bounded degree (given by the vanishing of a determinant).

\footnotetext{
${ }^{5}$ Even this can be relaxed to require only that $\langle A\rangle$ not be contained in the union $V$ of a bounded number of varieties of positive codimension and bounded degree, as is clear from the arguments we will see and as [BGT11] states explicitly. This boundedness condition is called "bounded complexity" in BGT11]. The "complexity" in BGT11] corresponds to the degree vector $\overrightarrow{\operatorname{deg}}(V)$ in Hel11.
} 
Since $G \times G$ is irreducible, and since we have shown that there is at least one point $\left(g_{1}^{\prime}, g_{2}^{\prime}\right)$ outside $W$, we see that all the components of $W$ have to be of positive codimension. Hence we can apply Lemma 5.1 (escape) with $A \times A$ (which generates $G \times G)$ instead of $A$ and $G \times G$ instead of $G$ and $V$, and obtain that there are $g_{1}, g_{2} \in A^{k^{\prime}}, k^{\prime}=O(1)$, such that $v, g_{1} v g_{1}^{-1}$ and $g_{2} v g_{2}^{-1}$ are linearly independent. This means that the derivative of $\phi$ at the origin $(e, e, e)$ of $T \times T \times T$ is nondegenerate when any such $g_{1}, g_{2} \in A^{k}$ are given.

The points of $T \times T \times T$ at which $\phi$ has degenerate derivative form, again, a subvariety $W_{0}$; since $T \times T \times T$ is irreducible, and since, as we have just shown, the origin $(e, e, e)$ does not lie on $W_{0}$, we see that $W_{0}$ is a union of components of positive codimension. This means that there is a subvariety $W_{1} \subset T \times T$ of bounded degree (bounded, mind you, independently of $g_{1}$ and $g_{2}$ ) made out of components of positive codimension, such that, for all $\left(t_{0}, t_{1}\right) \in T(K) \times T(K)$ not on $W_{1}$, there are $O(1)$ elements $t_{2} \in T(K)$ such that $\left(t_{0}, t_{1}, t_{2}\right)$ lies on $W_{0}$. We also see that there is a subvariety $W_{2} \subset T$ of bounded degree and positive codimension such that, for all $t_{0} \in T(K)$ not on $W_{2}$, there are $O(1)$ elements $t_{1} \in T(K)$ such that $\left(t_{0}, t_{1}\right)$ lies on $W_{1}$.

Given any point $y$ on $G(K)$, its preimage under the restriction $\left.\phi\right|_{(T \times T \times T) \backslash W_{0}}$ lies on a variety of dimension zero: if this were not the case, the preimage $\phi^{-1}(y)$ would be a variety $V_{y}$ such that there is at least one point $x$ not on $W_{0}$ lying on a component of positive dimension of $V_{y}$. There would then have to be a non-zero tangent vector to $V_{y}$ at $x$, and we see that its image under $D \phi$ would be 0 , i.e., $D \phi$ would be degenerate at $x$, implying that $x$ lies on $W_{0}$, a contradiction.

The preimage of $y$ under $\left.\phi\right|_{(T \times T \times T) \backslash W_{0}}$, besides being zero-dimensional, is also of bounded degree, because $\phi$ is of bounded degree. Hence the preimage consists of at most $C$ points, $C$ a constant.

Similarly, considering the boundedness of the degrees of $W_{0}, W_{1}$, and $W_{2}$, we see that there are at most $O(1)$ points $t_{0}$ on $W_{2}$, there are at most $|A \cap T(K)| \cdot O(1)$ points $\left(t_{0}, t_{1}\right) \in(A \cap T(K)) \times(A \cap T(K))$ on $W_{1}$ for $t_{0}$ not on $W_{2}$, and there are at most $|A \cap T(K)|^{2} \cdot O(1)$ points $\left(t_{0}, t_{1}, t_{2}\right) \in(A \cap T(K)) \times(A \cap T(K)) \times(A \cap T(K))$ on $W_{0}$ for $\left(t_{0}, t_{1}\right)$ not on $W_{1}$. Hence

$$
\left|\left\{x \in X: x \notin W_{0}(K)\right\}\right| \geq|A \cap T(K)|^{3}-O\left(|A \cap T(K)|^{2}\right)
$$

for $X=(A \cap T(K)) \times(A \cap T(K)) \times(A \cap T(K))$, and so

$$
\phi\left(\left\{x \in X: x \notin W_{0}(K)\right\} \mid\right) \geq \frac{|A \cap T(K)|^{3}-O\left(|A \cap T(K)|^{2}\right)}{C} .
$$

Since $\phi(X)$ lies in $A^{k}$ for $k=2 k^{\prime}+3$, we see that

$$
|A \cap T(K)|^{3}-O\left(|A \cap T(K)|^{2}\right) \leq C|A|
$$

and so $|A \cap T(K)|^{3} \ll\left|A^{k}\right|^{1 / 3}$.

The proof above follows [Hel11, Cor. 5.4], which establishes the same result for $\mathrm{SL}_{n}$ and indeed for all classical Chevalley groups. It is stated in more conceptual terms than the proof given in [Hel08, Lem. 4.7] (as might be expected). In consequence, it generalizes more easily; for instance, Hel11 carries out the same argument for non-maximal tori and unipotent subgroups. The main step was stated in general terms in Hel11, Prop. 4.12].

However, the situation was still not fully satisfactory. There is one passage in the proof of Proposition 5.2 that is not quite abstract enough: the one that starts with 
"It is easy to show". This is a very simple computation for $\mathrm{SL}_{2}$, and fairly easy even for $\mathrm{SL}_{n}$. The problem is that it has to be done from scratch for a specific algebraic group $G$ and a specific variety $V$ every time we want to generalize Proposition 5.2 . This means, first, that we have to go through Lie group types if we want a statement that is general on $G$, and that is tedious. Second, and more importantly, this kept the author from giving a statement for fully general $V$ in Hel11, as opposed to a series of statements for different varieties $V$.

A full generalization in these two senses was achieved independently by $\mathrm{PSa}$ and BGT11. It turns out that all we need to know about the algebraic group $G$ is that it is simple (or almost simple, like $\mathrm{SL}_{n}$ ). Under that condition, and assuming that $\langle A\rangle=G(K)$, Pyber and Szabó proved [PSa, Thm. 24] that, for every subvariety $V \subset G$ of positive dimension and every $\epsilon>0$,

$$
|A \cap V| \ll_{\epsilon}\left|A^{k}\right|^{(1+\epsilon) \frac{\operatorname{dim}(V)}{\operatorname{dim}(G)}},
$$

where $k$ and the implied constant depend only on $\epsilon$, on the degree and number of the components of $V$, and on the rank (and Lie type) of $G$. This they did by greatly generalizing and strengthening the arguments in [Hel11] (such as, for example, the proof of Lemma 5.2 above).

The route followed by Breuillard, Green, and Tao BGT11 was a little different. By then Hel11] was known - the first version was made public in 2008 - and the author had conversed at length with one of the authors of [BGT11] about the ideas involved and the difficulties remaining. The preprint of Larsen and Pink [LP11] was available, as were works by Hrushovski and Wagner [HW08] and Hrushovski [Hru12] 6 The aim of [LP11] was to give a classification of subgroups of GL without using the Classification Theorem of finite simple groups. This involved stating and proving (5.4) (without $\epsilon$ ) for $A$ a subgroup of $G(K)$ [LP11, Thm. 4.2]. This proof turned out to be robust (as Hrushovski and Wagner's model-theoretical work may have already indicated): BGT11] adapted it to the case of $A$ a set, obtaining (5.4) for every $A$ with $\langle A\rangle=G(K)$ (without the $\epsilon$, i.e., strengthened).

Rather than prove (5.4) here in full generality, we will prove in a case in which we need it. This is a case that, for $\mathrm{SL}_{2}$, would have been accessible by an approach even more concrete than that in [Hel08], as [Kow13] shows. However, the more conceptual proof below is arguably simpler, and also displays the main ideas in the proof of the general statement (5.4).

Proposition 5.3. Let $G=\mathrm{SL}_{2}$, and let $K$ be a field. Let $A \subset G(K)$ be a finite set with $A=A^{-1}, e \in A,\langle A\rangle=G$. Let $g$ be a regular semisimple element of $G(K)$. Then

$$
|A \cap \mathrm{Cl}(g)| \ll\left|A^{k}\right|^{2 / 3},
$$

where $k$ is an absolute constant.

For $G=\mathrm{SL}_{2}, g$ is regular semisimple if and only if it has two distinct eigenvalues. In that case, $\mathrm{Cl}(g)$ is just the subvariety $W$ of $G$ defined by $W=\{x: \operatorname{tr}(x)=\operatorname{tr}(g)\}$. (In general, in any linear algebraic group $G$, the conjugacy class of a semisimple element is a (closed) variety $\operatorname{Spr} 86$, Cor. 2.4.4].) Thus, $\operatorname{dim}(\mathrm{Cl}(g)) / \operatorname{dim}(G)=2 / 3$;

\footnotetext{
${ }^{6}$ Pyber and Szabó also mention the earlier paper [HP95] (Hrushovski and Pillay) as an influence. Part of the role of Hru12 was to make the work of Larsen and Pink clearer. According to LP11, p. 1108], there was actually a gap in the original version of [LP11], and Hru12] filled that gap, besides giving a more general statement.
} 
this is the meaning of the exponent in (5.5). The centralizer of a regular semisimple element is a torus.

The proof below is a little closer to [LP11] (apud BGT11) than to PSa, and also brings in some more ideas from Hel11. In both [LP11] and [PSa], recursion is used to reduce the problem to one for lower-dimensional varieties (not unlike what happens in the proof of Lemma 5.1) 7

Proof of Proposition 5.3. Write $Y$ for the variety $\overline{\mathrm{Cl}(g)}$. We start as in the proof of Proposition 5.2, defining a map $\phi: Y \times Y \rightarrow G$ by

$$
\phi\left(y_{0}, y_{1}\right)=y_{0} y_{1} \text {. }
$$

(We do not bother to conjugate as in (5.3) because $Y$ is invariant under conjugation; it is also invariant under inversion.) The preimage of a generic point of $G$ is not, unfortunately, zero dimensional, since $\operatorname{dim}(Y \times Y)=2 \cdot 2>3=\operatorname{dim}(G)$. Let $g \in \phi(Y \times Y)$. The preimage of $g$ is

$$
\left\{\left(y_{0}, y_{0}^{-1} g\right): y_{0} \in Y, y_{0}^{-1} g \in Y\right\}=\left\{\left(y_{0}, y_{0}^{-1} g\right): y_{0} \in Y \cap g Y\right\} .
$$

It is clear from this that the dimension of the preimage of $g$ equals $\operatorname{dim}(Y \cap g Y)$, and so there are at most two points with two-dimensional preimage, namely, $g=$ $\pm e \in G$. Assume $g \neq \pm e$.

By the usual $\mid$ domain $|\geq|$ image $\mid /$ largest preimage| argument, (5.7) implies

$$
\phi\left(\left\{\left(y_{0}, y_{1}\right) \in(A \cap Y) \times(A \cap Y): y_{1} \neq \pm y_{0}^{-1}\right\}\right) \geq \frac{|A \cap Y|(|A \cap Y|-2)}{\max _{g \neq \pm e}|A \cap Y \cap g Y|},
$$

and, since $\phi\left(\left\{\left(y_{0}, y_{1}\right) \in(A \cap Y) \times(A \cap Y)\right\}\right) \subset A^{2}$, we see that

$$
|A \cap Y| \leq 2+\sqrt{\left|A^{2}\right| \cdot \max _{g \neq \pm e}|A \cap Y \cap g Y|} .
$$

Hence, we should aim to bound $|A \cap Y \cap g Y|$ from above. The number of components of $Y \cap g Y$ is $O(1)$ because the degree of $Y$ and $g Y$ is bounded. Let $Z$ be the irreducible component of $Y \cap g Y$ containing the most elements of $A$. Since $g \neq \pm e$, $\operatorname{dim}(Z) \leq 1$; we can assume that $\operatorname{dim}(Z)=1$, as otherwise what we wish to prove is trivial. We want to bound $A \cap Z$. (This is the recursion we referred to before; we are descending to a lower-dimensional variety $Z$.)

We will now consider a map $\psi: Z \times Z \times Z \rightarrow G$ given by

$$
\psi\left(z_{0}, z_{1}, z_{2}\right)=z_{0} \cdot g_{1} z_{1} g_{1}^{-1} \cdot g_{2} z_{2} g_{2}^{-1} .
$$

Much as in the proof of Proposition 5.2. we wish to show that there are $g_{1}, g_{2} \in A^{k^{\prime}}$ such that, at all points of $Z \times Z \times Z$ outside a proper subvariety $W_{0}$ of $Z \times Z \times Z$, the derivative of $\psi$ is non-degenerate. Just as before, it will be enough to find a single point of $Z \times Z \times Z$ at which the derivative is non-degenerate. Choose any point $z_{0}$ on $Z$; we will look at the point $\left(z_{0}, z_{0}, z_{0}\right)$.

We write $\mathfrak{z}$ for the tangent space of $Z z_{0}^{-1}$ at the origin; it is a subspace of the Lie algebra of $G$. First, we compare the vector space $\mathfrak{z}$ (obtained by deriving $\psi\left(z, z_{0}, z_{0}\right) \psi\left(z_{0}, z_{0}, z_{0}\right)^{-1}$ at $\left.z=z_{0}\right)$ and the vector space $z_{0} g_{1} \mathfrak{z} g_{1}^{-1} z_{0}^{-1}$ (obtained by deriving $\psi\left(z_{0}, z, z_{0}\right) \psi\left(z_{0}, z_{0}, z_{0}\right)^{-1}$ at $\left.z=z_{0}\right)$. We would like them to be linearly

${ }^{7}$ The $\epsilon$ in (5.4) appears in $\mathrm{PSa}$ because the recursion there does not always end in the zero-dimensional case; rather, an excess concentration on a variety gets shuttled back and forth ("transport", PSa Lem. 27]) and augmented by itself a bounded number of times, until it is too large, yielding a contradiction and thereby proving (5.4). 
independent, i.e., intersect only at the origin; this is the same as asking whether there is a $g \in G(\bar{K})$ such that $\mathfrak{z}$ and $g \mathfrak{z} g^{-1}$ are linearly independent, since we can set $g_{1}=z_{0}^{-1} g$. If there were no such $g$, then $g \mathfrak{z} g^{-1}=\mathfrak{z}$ for all $g \in G(\bar{K})$, and so $\mathfrak{z}$ would be an ideal of the Lie algebra $\mathfrak{g}$ of $G$ (i.e., it would be invariant under the action ad of the Lie bracket). However, this is impossible, since a simple (or almost simple group of Lie type $G$ has a simple Lie algebra (prove as in [Bou72, III.9.8, Prop. 27]), i.e., an algebra $\mathfrak{g}$ without ideals other than itself and (0). Hence there is a $g_{1}$ such that $\mathfrak{z}$ and $z_{0} g_{1} \mathfrak{z} g_{1}^{-1} z_{0}^{-1}$ are linearly independent. This means that a determinant does not vanish at $g_{1}$. Thus we see that $\mathfrak{z}$ and $z_{0} g_{1} \mathfrak{z} g_{1}^{-1} z_{0}^{-1}$ are linearly independent for $g_{1}$ outside a subvariety $W$ that is a union of (a bounded number of) components of positive codimension (and bounded degree). If $|K|$ is larger than a constant, a simple bound on the number of points on varieties (weaker than either Lang-Weil or Schwartz-Zippel; [W54, Lem. 1] is enough) shows that $W(K) \neq G(K)$. We can assume that $|K|$ is larger than a constant, as otherwise the statement of the proposition we are trying to show is trivial. By escape from subvarieties (Lemma 5.1), it follows there is a $g_{1} \in A^{k}, k=O(1)$, lying outside $W$. Let us fix one such $g_{1}$.

We also want

$$
\left(x_{0} g_{1} x_{0} g_{1}^{-1}\right) g_{2} \mathfrak{z} g_{2}^{-1}\left(x_{0} g_{1} x_{0} g_{1}^{-1}\right)^{-1},
$$

obtained by deriving $\psi\left(z_{0}, z_{0}, z\right) \psi\left(z_{0}, z_{0}, z_{0}\right)^{-1}$ at $z=z_{0}$, to be linearly independent from $\mathfrak{z}+x_{0} g_{1} \mathfrak{z} g_{1}^{-1} x_{0}^{-1}$. This is done by exactly the same argument: $\mathfrak{z}+x_{0} g_{1} \mathfrak{z} g_{1}^{-1} x_{0}^{-1}$ cannot be an ideal of $\mathfrak{g}$ (because there are none, other than $\mathfrak{g}$ and $(0)$ ) and so there is a subvariety $W^{\prime} \subsetneq G$ (the union of a bounded number of components of positive codimension and bounded degree) such that the linear independence we want does hold for $g_{2}$ outside $W^{\prime}$. Again by a bound on the number of points, followed by escape (Lemma 5.1), there is a $g_{2} \in A^{k^{\prime}}, k^{\prime}=O(1)$, lying outside $W^{\prime}$.

Thus, we have $g_{1}, g_{2} \in A^{k^{\prime}}$ such that $\psi$ has a non-degenerate derivative at at least one point (namely, $\left.\left(z_{0}, z_{0}, z_{0}\right)\right)$. This means that $\psi$ has non-degenerate derivative outside a proper subvariety $W_{0} \subset Z \times Z \times Z$ (consisting of a bounded number of components of bounded degree). We finish exactly as in the proof of Proposition 5.2. using the same counting argument to conclude that

$$
\psi\left(\left\{x \in X: x \notin W_{0}(K)\right\} \mid\right) \gg|A \cap Z(K)|^{3}-O\left(|A \cap Z(K)|^{2},\right.
$$

and so $|A \cap Z(K)|^{3} \ll\left|A^{k}\right|^{1 / 3}$ for some $k=O(1)$. Substituting this into (5.8), we obtain that

$$
|A \cap Y| \ll 2+\sqrt{\left|A^{2}\right| \cdot\left|A^{k}\right|^{1 / 3}} \ll\left|A^{k}\right|^{2 / 3}
$$

for $A$ non-empty, as desired.

Remark. The proof of Proposition 5.3 lets itself be generalized fairly easily. In particular, note that we used no properties of $Z$ other than the fact that it was an irreducible one-dimensional variety of dimension 1 . Thus, we have actually shown that

$$
|A \cap Z(K)| \ll\left|A^{k}\right| \frac{1}{\operatorname{dim}(G)}
$$

for any one-dimensional irreducible subvariety of a simple (or almost simple) group of Lie type $G$; the implied constant depends only on the degree of $Z$, the dimension

\footnotetext{
${ }^{8}$ Meaning that $G$ has no connected normal algebraic subgroups other than itself and the identity; this is the case for $G=\mathrm{SL}_{2}$.
} 
of $G$, and the degree of the group operation in $G$ as a morphism. The main way in which the proof was easier than a full proof of

$$
|A \cap V| \ll\left|A^{k}\right|^{\frac{\operatorname{dim}(V)}{\operatorname{dim}(G)}}
$$

is that, since we were dealing with low-dimensional varieties, the inductive process was fairly simple, as were some of the counting arguments. However, the basic idea of the general inductive process is the same as here - go down in dimension, keeping the degree under control. It is not necessary for the maps used in the proof to be roughly injective (like the map $\phi$ in (5.3) ), as long as the preimage of a generic point is a variety whose dimension is smaller than the dimension of the domain (as is the case for the map $\phi$ in (5.6) ). This means, in particular, that we need not try to make the Lie algebras $\mathfrak{z}, g \mathfrak{z} g^{-1}$ be linearly independent-it is enough to ask that $g \mathfrak{z} g^{-1}$ not be equal to $\mathfrak{z}$ (clearly a weaker condition when $\operatorname{dim}(\mathfrak{z})>1$ ). We get $g \mathfrak{z} g^{-1} \neq \mathfrak{z}$ easily by the same argument as in the proof of Proposition 5.3 , using the simplicity of $G$.

We can now use the orbit-stabilizer theorem for sets to convert the upper bound given by Proposition 5.3 into a lower bound for $\left|A^{2} \cap T(K)\right|$. The idea is that having many elements of $A^{2}$ (or $A^{k}$ ) of a special form (e.g., elements of $T(K)$ ) is extremely useful.

Corollary 5.4. Let $G=\mathrm{SL}_{2}$, and let $K$ be a field. Let $A \subset G(K)$ be a finite set with $A=A^{-1}, e \in A, A \subset G(K)$. Let $g$ be a regular semisimple element of $A^{l}$, $l \geq 1$. Assume $\left|A^{3}\right| \leq|A|^{1+\delta}, \delta>0$. Then

$$
\left|A^{2} \cap C(g)\right| \gg|A|^{\frac{1}{3}-O(\delta)},
$$

where $k$ and the implied constants depend only on $l$.

This corresponds to LP11, Thm. 6.2]. The centralizer $C(g)$ is a maximal torus T. Clearly, $1 / 3=\operatorname{dim}(T) / \operatorname{dim}(G)$.

Proof. By Proposition 5.3 and Lemma 4.5 (orbit-stabilizer),

$$
\left|A^{2} \cap C(g)\right| \gg \frac{A}{\left|A^{k l}\right|^{2 / 3}} .
$$

The inequality $\left|A^{k l}\right| \ll|A|^{1+O(\delta)}$ follows from $\left|A^{3}\right| \leq|A|^{1+\delta}$ and (3.3).

This has been a centerpiece of proofs of growth in $\mathrm{SL}_{2}$ - and linear algebraic groups in general-since Hel08]. At the same time, there is a precision to be made. Proposition 4.1 in that paper proved Corollary 5.4 for some $g \in A$ (i.e., there exists a torus $T=C(g)$ with a large intersection with $\left.A^{2}\right)$. The same proof given there shows that this is true for most $g \in A$, but it does not prove it for all $g \in A$. This was, in retrospect, an important technical weakness. It made the rest of the argument more indirect and harder to generalize. This remained the case when Corollary 5.4 was itself stated in greater generality in Hel11, Prop. 5.8 and Cor. 5.10]. 
5.3. High multiplicity and spectral gaps, I. In order to supplement our main argument, we will need to be able to show that, if $A$ is very large $\left(|A|>|G|^{1-\delta}\right.$, $\delta>0$ small), then $\left(A \cup A^{-1} \cup\{e\}\right)^{k}=G$. (See the statement of Theorem [1.1.) This task is not particularly hard; in Hel08, it was done "by hand", using a descent to a Borel subgroup and results on large subsets of $\mathbb{F}_{p}$. As Nikolov and Pyber later pointed out, one can obtain a stronger result (with $k=3$ ) in a way that generalizes very easily. This requires a key concept - that of high eigenvalue multiplicity — which will appear again in $\$ 5.5$

Proposition 5.5 (Frobenius). Let $G=\mathrm{SL}_{2}\left(\mathbb{F}_{q}\right), q=p^{\alpha}, p$ odd. Then every non-trivial complex representation of $G$ has dimension at least $(q-1) / 2$.

It is of course enough to show that every irreducible non-trivial complex representation has dimension at least $(q-1) / 2$.

Proof. This can be seen directly from, e.g., the character tables in [FH91, §5.2]. See also the standard reference [LS74] or the exposition in [DSV03, §3.5] (for $G=$ $\mathrm{PSL}_{2}\left(\mathbb{F}_{p}\right), p$ prime).

There are analogues of Proposition 5.5 for all finite simple groups of Lie type.

Now consider a Cayley graph $\Gamma(G, A)$, where $A$ generates $G$ and $A=A^{-1}$. We recall that this is defined to be the graph having $G$ as its set of vertices and $\{(g, a g): g \in G, a \in A\}$ as its set of edges. We recall that the normalized adjacency matrix $\mathscr{A}$ is a linear operator on the space $L$ of complex-valued functions on $G$ : it is defined by

$$
(\mathscr{A} f)(g)=\frac{1}{|A|} \sum_{a \in A} f(a g) .
$$

Since $A$ is symmetric, $\mathscr{A}$ has a full real spectrum

$$
\cdots \leq \lambda_{2} \leq \lambda_{1} \leq \lambda_{0}=1
$$

with orthogonal eigenvectors $v_{j}$; the eigenvector $v_{0}$ corresponding to the highest eigenvalue $\lambda_{0}$ is just a constant function.

We can see from the definition (5.9) that every eigenspace of $\mathscr{A}$ is invariant under the action of $G$ on the right; in other words, it is a representation-and it can be trivial only for the eigenspace consisting of constant functions, i.e., the eigenspace associated to $\lambda_{0}$. Hence, by Proposition [5.5] every eigenvalue $\lambda_{j}, j>0$, has multiplicity at least $(q-1) / 2$.

The idea now is to use this high multiplicity to show a spectral gap, i.e., a nontrivial upper bound for $\lambda_{1}$. Let us follow Gow08, which shows that this is not hard for $A$ large. The trace of $\mathscr{A}^{2}$ can be written in two ways: on one hand, it is $1 /|A|^{2}$ times the number of length-2 paths whose head equals their tail, and, on the other hand, it equals a sum of squares of eigenvalues. In other words,

$$
\frac{|G||A|}{|A|^{2}}=\sum_{j} \lambda_{j}^{2} \geq \frac{q-1}{2} \lambda_{j}^{2}
$$

for every $j \geq 1$, and so

$$
\left|\lambda_{j}\right| \leq \sqrt{\frac{|G| /|A|}{\frac{q-1}{2}}} .
$$


If $A$ is large enough (close to $|G|$ in size), this is much smaller than $\lambda_{0}=1$. This means that a few applications of $\mathscr{A}$ "uniformize" any distribution very quickly, in that anything orthogonal to a constant function gets multiplied by $\lambda_{1}<1$ (or less) repeatedly. The proof of the following result is based on this idea.

Proposition 5.6 (Gow08] and [NP11]). Let $G=\mathrm{SL}_{2}\left(\mathbb{F}_{q}\right)$, and let $q$ be an odd prime power. Let $A \subset G, A=A^{-1}$. Assume $|A| \geq 2|G|^{8 / 9}$. Then

$$
A^{3}=G \text {. }
$$

Neither Gow08 nor [NP11] require $A=A^{-1}$; we are assuming it for simplicity.

Proof. Suppose there is a $g \in G$ such that $g \notin A^{3}$. Then the inner product $\left\langle\mathscr{A} 1_{A}, 1_{g A^{-1}}\right\rangle$ equals 0 . We can assume that eigenvectors $v_{j}$ have $\ell_{2}$-norm 1 (relative to the counting measure on $G$, say). Then

$$
\begin{aligned}
& \left\langle\mathscr{A} 1_{A}, 1_{g A^{-1}}\right\rangle=\lambda_{0}\left\langle 1_{A}, v_{0}\right\rangle\left\langle v_{0}, 1_{g A^{-1}}\right\rangle+\sum_{j \geq 1} \lambda_{j}\left\langle 1_{A}, v_{j}\right\rangle\left\langle v_{j}, 1_{g A^{-1}}\right\rangle \\
& =|A| \cdot\left(\frac{|A|}{\sqrt{|G|}}\right)^{2}+O^{*}\left(\sqrt{\frac{2|G||A|}{q-1}} \sqrt{\sum_{j \geq 1}\left|\left\langle 1_{A}, v_{j}\right\rangle\right|^{2}} \sqrt{\sum_{j \geq 1}\left|\left\langle v_{j}, 1_{g A^{-1}}\right\rangle\right|^{2}}\right) \\
& =\frac{|A|^{3}}{|G|}+O^{*}\left(\sqrt{\frac{2|G||A|}{q-1}}\left|1_{A}\right|_{2}\left|1_{g A^{-1}}\right|_{2}\right)=\frac{|A|^{3}}{|G|}+O^{*}\left(\sqrt{\frac{2|G||A|}{q-1}}|A|\right) .
\end{aligned}
$$

By $|G|=\left(q^{2}-q\right) q$, however, $|A| \geq 2|G|^{8 / 9}$ implies $|A|^{3} /|G|>\sqrt{2|G||A| /(q-1)}|A|$, and so (5.11) means that $\left\langle\mathscr{A} 1_{A}, 1_{g A^{-1}}\right\rangle$ cannot be 0 .

5.4. Growth in $\mathrm{SL}_{2}\left(\mathbb{F}_{q}\right)$. We finally come to the proof of Theorem 1.1. This is a "modern" proof, without any reliance on the sum-product theorem, and with a fairly straightforward generalization to higher-rank groups. This part is a little closer to $\mathrm{PSa}$ ] than to the first version of [BGT11. Note the parallels with Proposition 4.7 (pivoting).

We will actually be able to prove Theorem 1.1 over a general finite field $\mathbb{F}_{q}$, and not just over $\mathbb{F}_{p}$. The statement is as follows.

Theorem 5.7. Let $G=\mathrm{SL}_{2}\left(\mathbb{F}_{q}\right)$. Let $A \subset G$ generate $G$. Then either

$$
\left|A^{3}\right| \geq|A|^{1+\delta}
$$

or $\left(A \cup A^{-1} \cup\{e\}\right)^{k}=G$, where $\delta>0$ and $k \geq 1$ are absolute constants.

Proof. By (3.2), we can assume that $A=A^{-1}$ and $e \in A$ without loss of generality. We can also assume $A$ to be larger than an absolute constant, as otherwise $\left|A^{2}\right| \geq$ $|A|+1$ gives us $\left|A^{3}\right| \geq|A|^{1+\delta}$ trivially. (If $\left|A^{2}\right| \leq|A|$, then $A^{2} \supset A \cdot e=A$ implies $A^{2}=A$, and, since $A$ generates $G, A \cdot A=A$ implies that $A=G$.)

Assume $\left|A^{3}\right|<|A|^{1+\delta}$, where $\delta>0$ will be set later. By Lemma 5.1 (escape), there is an element $g_{0} \in A^{2}$ that is regular semisimple (that is, $\operatorname{tr}\left(g_{0}\right) \neq \pm 2$ ). Its centralizer $T=C_{G}\left(g_{0}\right)$ is a maximal torus.

Call $\xi \in G$ a pivot if the function $\phi_{\xi}: A \times T \rightarrow G$ defined by

$$
(a, t) \mapsto a \xi t \xi^{-1}
$$

is injective when considered as a function from $A /\{ \pm e\} \times T /\{ \pm e\}$ to $G /\{ \pm e\}$. (The analogy with the proof of Proposition 4.7 is deliberate.) 
Case (a): There is a pivot $\xi$ in $A$. Since $T=C\left(g_{0}\right)$, Corollary 5.4 (together with $\left.\left|A^{k}\right| \leq|A|^{1+O(\delta)}\right)$ gives us that there are $\gg|A|^{1 / 3-O(\delta)}$ elements of $T$ in $A^{2}$. Hence, by the injectivity of $\phi_{\xi}$,

$$
\left|\phi_{\xi}\left(A, A^{2} \cap T\right)\right| \geq \frac{1}{4}|A|\left|A^{2} \cap T\right| \gg|A||A|^{\frac{1}{3}-O(\delta)}=|A|^{\frac{4}{3}-O(\delta)} .
$$

At the same time, $\phi_{x}\left(A, A^{2} \cap T\right) \subset A^{5}$, and so

$$
\left|A^{5}\right| \gg|A|^{4 / 3-O(\delta)} \text {. }
$$

For $\delta$ smaller than a positive constant, this gives a contradiction to $\left|A^{3}\right|<|A|^{1+\delta}$ by Ruzsa's inequality (3.3). (Recall that we can assume that $|A|$ is larger than an absolute constant.)

Case (b): There are no pivots $\xi$ in $G$. Then, for every $\xi \in G$, there are $a_{1}, a_{2} \in A$, $t_{1}, t_{2} \in T,\left(a_{1}, t_{1}\right) \neq\left( \pm a_{2}, \pm t_{2}\right)$ such that $a_{1} \xi t_{1} \xi^{-1}= \pm e \cdot a_{2} \xi t_{2} \xi^{-1}$, i.e.,

$$
a_{2}^{-1} a_{1}= \pm e \cdot \xi t_{2} t_{1}^{-1} \xi^{-1} \text {. }
$$

In other words,

$$
A^{-1} A \cap \xi T \xi^{-1} \neq\{ \pm e\}
$$

for every $\xi \in G$.

Choose any $g \in \xi T \xi^{-1}$ with $g \neq \pm e$. Then $g$ is regular semisimple and its centralizer $C(g)$ equals $\xi T \xi^{-1}$. (This is particular to $\mathrm{SL}_{2}$; see the comments after the proof.) Thus, by Corollary 5.4, we obtain that there are $\geq c|A|^{1 / 3-O(\delta)}$ elements of $\xi T \xi^{-1}$ in $A^{k}$, where $k$ and $c>0$ are absolute. This is valid for every conjugate $\xi T \xi^{-1}$ of $T$ with $\xi \in G$. At least $(1 / 2)|G| /|T|$ maximal tori of $G$ are of the form $\xi T \xi^{-1}, \xi \in G$. Hence

$$
\left|A^{k}\right| \geq \frac{1}{2} \frac{|G|}{|T|}\left(c|A|^{1 / 3-O(\delta)}-2\right) \gg p^{2}|A|^{1 / 3-O(\delta)} .
$$

(Since any element of $G$ other than $\pm e$ can lie on at most one maximal torus, there is no double counting.)

From (5.14) it follows immediately that either $\left|A^{3}\right| \geq|A|^{1+\delta}$ (use (3.3)) or $A \geq$ $|G|^{1-O(\delta)}$. In the latter case, Proposition [5.6 implies that $A^{3}=G$.

Case (c): There are pivots and non-pivots in $G$. Since $\langle A\rangle=G$, this implies that there is a $\xi \in G$, not a pivot, and an $a \in A$ such that $a \xi$ is a pivot. Since $\xi$ is not a pivot, we obtain (5.13), and so there are $\geq|A|^{1 / 3-O(\delta)}$ elements of $\xi T \xi^{-1}$ in $A^{k}$, just as before. Hence

At the same time, $a \xi$ is a pivot, i.e., the map $\phi_{a \xi}$ (defined in (5.12) ) is injective.

$$
\left|\phi_{a \xi}\left(A, \xi^{-1}\left(A^{k} \cap \xi T \xi^{-1}\right) \xi\right)\right| \geq \frac{1}{4}|A|\left|A^{k} \cap \xi T \xi^{-1}\right| \geq \frac{1}{4}|A|^{\frac{4}{3}-O(\delta)} .
$$

Since $\phi_{a \xi}\left(A, \xi^{-1}\left(A^{k} \cap \xi T \xi^{-1}\right) \xi\right) \subset A^{k+3}$, it follows that

$$
\left|A^{k+3}\right| \geq \frac{1}{4}|A|^{4 / 3-O(\delta)} .
$$

We set $\delta$ small enough for Ruzsa's inequality (3.3) to imply that (5.15) contradicts $\left|A^{3}\right| \leq|A|^{1+\delta}$. 
One apparent obstacle to a generalization here is the fact that, in higher-rank groups (e.g., $\mathrm{SL}_{n}, n \geq 3$ ), the centralizer $C(g)$ of an element $g \neq \pm e$ of a torus $T$ is not necessarily equal to $T$; we have $C(g)=T$ only when $g$ is regular. This obstacle is not serious here, as the number of non-regular elements of $A$ on a torus is small by a dimensional bound; this is already in [Hel11, §5.5]. The difficulty in generalizing Theorem 1.1 to higher-rank groups (Hel11, GH11]) resided, in retrospect, in the fact that the version of Corollary 5.4 in [Hel08, §4] and [Hel11, Cor. 5.10] was slightly weaker, as discussed before. This made the pivoting argument more complicated and indirect, and thus harder to generalize; in particular, the sum-product theorem was still used, in spite of the attempts to gain independence from it in [Hel11, §3]. These issues were resolved in both $\mathrm{PSa}$ and BGT11.

As pointed out in BGT11, Theorem 1.1 actually implies the sum-product theorem; however, it is arguably more natural to deduce the sum-product theorem, or Proposition 3.7, from growth in the affine group (Proposition 4.7); multiplication and addition correspond to two different group actions; see 33.3 .

Let us now briefly go over the successive generalizations of Theorem $1.1 \mathrm{up}$ to this point. This will not just be a way to mention a series of contributions by different people in the field, it will also be a good opportunity to make a few additional points on the internal structure of the subject.

There are two main kinds of generalizations: changing the field and changing the Lie type.

Changing the field. Dinai Din11] showed that Theorem 1.1] holds with $\mathbb{F}_{p}$ changed to a general finite field $\mathbb{F}_{q}$. At the time, this involved some careful work involving the subgroup structure of $\mathrm{SL}_{2}\left(\mathbb{F}_{q}\right)$. Varjú Var12] gave a second, simpler proof of the same generalization.

The proof in Hel08 easily gives that Theorem 1.1 still holds if $\mathbb{F}_{p}$ is changed to $\mathbb{C}$ and $A$ is taken to be finite. In fact, in that case, there is a predecessor: Elekes and Király proved [EK01] a result corresponding to Theorem [1.1 with $\mathbb{R}$ instead of $\mathbb{F}_{p}$, with unspecified growth bounds. In general, in arithmetic combinatorics, results over $\mathbb{R}$ or $\mathbb{C}$ are more accessible than results over $\mathbb{F}_{p}: \mathbb{R}$ has an ordering and a topology that a general field, or $\mathbb{F}_{p}$ in particular, does not have. See the discussion on the sum-product theorem at the beginning of $\$ 3.3$ for a relevant instance of this.

Equally important is the fact that the new growth bounds on non-commutative groups are quantitatively strong $\left(\left|A^{3}\right| \geq|A|^{1+\delta}\right)$. Of course, one can take advantage of the structure of $\mathbb{R}$ or $\mathbb{C}$ and also give quantitatively strong estimates. A case in point is [Cha08, which gives (a) a simplified proof over $\mathbb{C}$ (based on Hel08]) and (b) an early attack on $\mathrm{SL}_{3}$.

It turns out that, for applications, one needs a stronger generalization to groups over $\mathbb{C}$ : a proof of expansion requires results for measures, and not just for sets $A$. If we are working over a finite field, then results of type $\left|A^{3}\right| \geq|A|^{1+\delta}$ for finite sets $A$ and results of type $|\mu * \mu|_{2} \leq|\mu|^{1+\delta}$ for measures $\mu$ on $G$ (with $|\mu|_{1}=1$ ) are equivalent (as was already pointed out in $[\mathrm{BG08 \textrm {C }}$ ); however, over $\mathbb{R}$ or $\mathbb{C}$, results for measures $\mu$ are stronger than results for sets $A$. It is in the context of measures that the new ideas we are now discussing become as crucial over infinite fields as they are over finite fields.

The first result for measures was proven in BG08b] for $\mathrm{SU}(2)$, which has the same Lie algebra type as $\mathrm{SL}_{2}$ : the main idea is to redo the proof in Hel08, still for finite sets, but keeping track of distances (e.g., where Hel08 uses that a map 
is injective, BG08b] also checks that the map does not shrink distances by more than a constant factor).

Changing the Lie type. The generalization of Theorem 1.1 to $\mathrm{SL}_{3}\left(\mathbb{F}_{p}\right)$ Hel11] was neither easy nor limited to $\mathrm{SL}_{3}$ alone; it involved general work on tori, conjugacy classes and slowly growing sets. It was also then that ideas from sum-product theorems (pivoting; see \$5) were taken to the context of actions of groups on groups.

Part of the generalization to $\mathrm{SL}_{n}$ was carried out in GH11, but, for instance, $\mathrm{SO}_{n}$ ( $n$ large) resisted (and was an obstruction to a full solution for $\mathrm{SL}_{n}$ ). Full and elegant generalizations to all finite simple groups of Lie type (with bounds depending on the rank) were given by Pyber and Szabó [PSa] and, independently, by Breuillard, Green, and Tao [BGT11] this, of course, covered the classical groups $\mathrm{SL}_{n}\left(\mathbb{F}_{q}\right), \mathrm{SO}_{n}\left(\mathbb{F}_{q}\right), \mathrm{Sp}_{2 n}\left(\mathbb{F}_{q}\right)$, with $\delta>0$ depending on $n$. (The issue of the dependence on $n$ is important; we will discuss it in some detail later.)

The case in some sense opposite to that of simple groups is that of solvable groups. We went over the case of a small but paradigmatic solvable group in detail - the affine group of $\mathbb{P}^{1}(4.2)$. The general case of solvable subgroups of $\mathrm{SL}_{n}\left(\mathbb{F}_{p}\right)$ is treated in $[\mathrm{GH}]$. A clean generalization of $\left.\mathrm{GH}\right]$ to $\mathbb{F}_{q}$ still remains to be done.

5.5. High multiplicity and spectral gaps, II. Now that we have proven the main theorem (Theorem 1.1), we may as well finish our account of growth in linear groups by going briefly over the proof of Theorem 1.3 (Bourgain and Gamburd), which gives us expanders. We will keep an eye on how the proof (from [BG08c]) can be adapted to general $G$.

In $₫ 2$, we said a pair $\left(G, A_{G}\right)$ gives an $\epsilon$-expander if every $S \subset G$ with $|S| \leq|G| / 2$ satisfies $\left|S \cup A_{G} S\right| \geq(1+\epsilon)|S|$. We also gave an alternative definition, based on eigenvalues; phrased in terms of the normalized adjacency matrix $\mathscr{A}$ on the Cayley graph $\Gamma(G, A)$ (see (5.9)), it states that a graph is an $\epsilon$-expander if the second largest eigenvalue $\lambda_{1}$ of $\mathscr{A}$ is at most $1-\epsilon$. Being an $\epsilon$-expander according to the second definition implies being an $(\epsilon / 2)$-expander according to the first definition: if $\left|S \cup A_{G} S\right|<(1+\epsilon / 2)|S|$, then $f: G \rightarrow \mathbb{C}$ defined by $f(x)=1_{S}-|S| /|G|$ would obey $\langle\mathscr{A} f, f\rangle>(1-\epsilon)\langle f, f\rangle$ and $\left\langle f, 1_{G}\right\rangle=0$, a contradiction if $\lambda_{1} \leq 1-\epsilon$. The two definitions are, in fact, equivalent for $|A|$ bounded; if the pair $\left(G, A_{G}\right)$ gives us an $\epsilon$ expander in the first sense, then the second largest eigenvalue $\lambda_{1}$ of $\mathscr{A}$ on $\Gamma\left(G, A_{G}\right)$ satisfies $\lambda_{1} \leq 1-\epsilon^{2} / 2|A|^{2}$ (see, e.g., [DSV03, Thm. 1.2.3] or [LPW09, §13.3.2]).

We will now prove Theorem 1.3, which states that, for $A \subset \mathrm{SL}_{2}(\mathbb{Z}),\langle A\rangle$ Zariskidense, $\left\{\Gamma\left(\mathrm{SL}_{2}(\mathbb{Z} / p \mathbb{Z}), A \bmod p\right)\right\}_{p}$ prime is a family of expander graphs. We will be using the second definition, that is, we will show that $\lambda_{1} \geq 1-\epsilon$, where $\epsilon>0$ depends only on $A$, not on $p$.

We recall at this point what was known before on expander families in $\mathrm{SL}_{2}(\mathbb{Z} / p \mathbb{Z})$, even though we will not use those previous results. As we said in $₫ 2$ it was known that the existence of the Selberg spectral gap [Sel65] on the surface $\Gamma(N) \backslash \mathbb{H}$ implies that all pairs $\left(\mathrm{SL}_{2}(\mathbb{Z} / p \mathbb{Z}), A \bmod p\right)$ with

$$
A=\left\{\left(\begin{array}{ll}
1 & 1 \\
0 & 1
\end{array}\right),\left(\begin{array}{ll}
1 & 0 \\
1 & 1
\end{array}\right)\right\}
$$

are $\epsilon$-expanders for some fixed $\epsilon>0$; the same holds for all other $A \subset \mathrm{SL}_{2}(\mathbb{Z})$ such that $\langle A\rangle$ is of finite index in $\mathrm{SL}_{2}(\mathbb{Z})$. The implication is not, from today's 
perspective, particularly difficult, but tracing the first time it was remarked is nontrivial; what follows is a preliminary attempt at a one-paragraph history of the implication, since this seems to be a matter of general interest in the field.

The implication was shown for compact quotients of $\mathbb{H}$ in Buser's work Bus78. See also Brooks Bro86. (still for the compact case) and Bro87. (non-compact case, in terms of the Kazhdan constant) and Burger Bur86. (compact case, in terms of eigenvalues). What is a little harder to pinpoint is the first proof for the non-compact case (in particular, $\Gamma(N) \backslash \mathbb{H}$ ) in terms of eigenvalues. (At least some proofs for the compact case do generalize to the non-compact case - see, e.g., [EHK12, App. A], based on Burger's approach - but this seems not to have been obvious at first.) A. Lubotzky and P. Sarnak (private communication) state that the work leading to $[$ LPS88 $]$ was originally centered on $\operatorname{SL}(\mathbb{Z} / p \mathbb{Z})$ and $\Gamma(N) \backslash \mathbb{H}$ and, in particular, showed the correspondence in this (non-compact) case. Thanks are due to them and to E. Kowalski for several references.

Let us go back to the proof of Theorem 1.3. which is a much more general statement, as it works for $A \subset \mathrm{SL}_{2}(\mathbb{Z})$ essentially arbitrary. (We recall that the only condition is that $\langle A\rangle$ be Zariski-dense in $\mathrm{SL}_{2}(\mathbb{Z})$; this is roughly what is necessary for $A \bmod p$ to generate $\mathrm{SL}_{2}(\mathbb{Z} / p \mathbb{Z})$ in the first place.)

Let us first sketch, as we promised, a proof of the weaker statement that $G_{p}$ has logarithmic diameter $O_{A}(\log |G|)$ with respect to $A_{p}$. Let us assume, for simplicity, that $A$ generates a free group, i.e., any two products $a_{1} \cdots a_{k}, a_{1}^{\prime} \cdots a_{k^{\prime}}^{\prime}$ of elements of $A \cup A^{-1}$ are distinct, unless they are equal for the trivial reason of having the same reduction (e.g., $x x^{-1} y z=y w^{-1} w z$, since both reduce to $y z$ purely formally). (The condition that $A$ generates a free group is fulfilled both by (2.1) and by (2.2); in general, the argument works because, by the Tits alternative, a subset of $\mathrm{SL}_{n}$ generating a Zariski-dense group must contain a pair of elements generating a free group. For $n=2$, as we will soon see, the Tits alternative is easy to prove by the use of the free group $\Gamma(2)$.)

If $k$ and $k^{\prime}$ are $<\log _{c}(p / 2)$ (where $c=2 \cdot 3=6$ for $A$ is in (2.2)), then $a_{1} \cdots a_{k} \neq a_{1}^{\prime} \cdots a_{k^{\prime}}^{\prime}$ implies $a_{1} \cdots a_{k} \not \equiv a_{1}^{\prime} \cdots a_{k^{\prime}}^{\prime} \bmod p$, simply because the entries of the matrices involved are $<p / 2$ in absolute value, and thus cannot be congruent without being equal. Hence, for $k=\left\lfloor\log _{c} p / 2\right\rfloor,\left|\left(A_{p} \cup A_{p}^{-1} \cup e\right)^{k}\right|$ is already quite large $\left(\geq(2|A|-1)^{k} \geq p^{\delta_{c}}, \delta_{c} \gg 0\right)$ 9 We can then apply Theorem 1.1 a bounded number of times and conclude that the diameter of $G_{p}$ with respect to $A_{p}$ is in fact $\ll \log p \ll \log |G|$, i.e., logarithmic.

As we will now see, the proof of Bourgain and Gamburd's expansion theorem is considerably more difficult.

Proof of Theorem 1.3. Let $S=A \cup A^{-1} \cup\{e\}$. Let $G=\mathrm{SL}_{2}$. Let $\mu$ be the measure on $G_{p}=\mathrm{SL}_{2}(\mathbb{Z} / p \mathbb{Z})$ given by

$$
\mu(x)= \begin{cases}\frac{1}{|S|} & \text { if } x \in S \\ 0 & \text { otherwise }\end{cases}
$$

\footnotetext{
${ }^{9}$ This bound on growth in the free group is trivial: given a word ending in, say, $x$, we can choose to prolong it by any element of $A \cup A^{-1}$ other than $x^{-1}$. Note, however, that obtaining a result like Theorem 1.1 for the free group is far from trivial; Theorem 1.1 (and [Cha08]) imply such a result, but the first direct proof is due to Razborov Raz14, who proved a strong bound $\left|A^{3}\right| \geq|A|^{2} /(\log |A|)^{O(1)}$ for any finite subset $A$ of a free group on at least two elements.
} 
We consider the convolutions $\mu^{(k)}=\mu * \mu * \cdots * \mu$. We will see how $\left|\mu^{(k)}\right|_{2}$ decreases as $k$ increases. This happens very quickly at first (Stage 1). It then goes on happening quickly enough (Stage 2), thanks to Theorem 1.1 (applied via Proposition 3.5, the Bourgain-Gamburd "flattening lemma"). Once $\left|\mu^{(k)}\right|_{2}$ is quite small (not much larger than $1 /\left|G_{p}\right|$, which is the least it could be), the proof can be finished off by an argument from [SX91], based on the same high-multiplicity phenomenon that was exploited in $\$ 5.3$.

Stage 0 (Reduction to $\langle A\rangle$ free). For $G=\mathrm{SL}_{2}$, we can (as in BG08c]) define $H=\Gamma(2)=\{g \in G(\mathbb{Z}): g \equiv I \bmod 2\}$. Now, $H$ is both free and of finite index in $G(\mathbb{Z})$; hence $\langle A\rangle \cap H$ is free (since, by the Nielsen-Schreier theorem, every subgroup of a free group is free), Zariski-dense, and generated by a set $A^{\prime} \subset\langle A\rangle$ of bounded size (Schreier generators). We can thus replace $A$ by $A^{\prime}$ (at the cost of at most a constant factor-depending on $A$ and $A^{\prime}$-in the final bounds) and assume from now on that $\langle A\rangle$ is free.

(For general $G$, the task is much more delicate, since such a convenient $H$ does not in general exist, and also because the "concentration in subgroups" issue we will discuss below requires stronger inputs to be addressed successfully-Zariski density no longer seems enough (given current methods). See GV12 for a general solution. An approach via products of random matrices is also possible BG08a, [BG09.)

Stage 1 (Decrease in $\left|\mu^{(k)}\right|_{2}$ for $k \ll \log |G|$, due to freedom). We can now assume that $\langle A\rangle$ is a free group on $r \geq 2$ elements. By the argument we went over in the introduction (shortly before the statement of Theorem 1.3), there is a constant $c$ depending only on $A$ such that two words on $A$ of length $k \leq c \log p$ reduce to the same element of $G(\mathbb{Z} / p \mathbb{Z})$ only if they give the same element of $G(\mathbb{Z})$; since $\langle A\rangle$ is free, this can happen only if they have the same reduction (e.g., $\left.x x^{-1} y z=x w^{-1} w z\right)$. Thus, for instance,

$$
\mu^{(k)}(e)=\frac{\mid \text { words of length } k \text { reducing to the identity } \mid}{r^{k}},
$$

where $\mu^{(k)}=\mu * \mu * \cdots * \mu$ ( $k$ times). Hence, Kesten's bound on the number of words of given length reducing to the identity [Kes59] gives us that, for any $\epsilon>0$,

$$
\mu^{k}(e) \ll_{\epsilon}\left(\sqrt{\frac{2 r-1}{r}}+\epsilon\right)^{k},
$$

and so, for $k=\lfloor c \log p\rfloor$,

$$
\mu^{(k)}(e) \ll \frac{1}{p^{\eta}},
$$

where $\eta>0$ depends only on $c$, and thus only on $A$. (Note that, if $r \geq 3$, the simple bound [BS87b, Lem. 2] can be used instead of Kesten's result.)

It turns out that, using the fact that $\langle A\rangle$ is free, we can show not just that $\mu^{(k)}(e)$ is small, but that $\mu^{(k)}\left(G^{\prime}\right)$ is small for any proper subgroup $G^{\prime}$ of $G$. For $G=\mathrm{SL}_{2}$, this is relatively straightforward: every proper subgroup $G^{\prime}$ of $G_{p}=\mathrm{SL}_{2}(\mathbb{Z} / p \mathbb{Z})$ is almost solvable, i.e., contains a solvable subgroup $G^{\prime \prime}$ of bounded index. It is enough to show that $\mu^{(2 k)}\left(G^{\prime \prime}\right)$ is small (as this implies immediately that $\mu^{(k)}\left(G^{\prime}\right)$ 
is small, by pigeonhole). Because we are in $\mathrm{SL}_{2}, G^{\prime \prime}$ is not just solvable but 2-step solvable, i.e., any elements $g_{1}, g_{2}, g_{3}, g_{4} \in G^{\prime \prime}$ must satisfy

$$
\left[\left[g_{1}, g_{2}\right],\left[g_{3}, g_{4}\right]\right]=e \text {. }
$$

By the same idea as before, for $k \leq c \log p, c$ small enough, this is possible only if $g_{1}, g_{2}, g_{3}, g_{4}$ are projections $\bmod p$ of elements of $\langle A\rangle$ that also satisfy (5.17). However, any set $S$ of words of length $\leq l$ in a free group such that all 4 -tuples of elements of $S$ satisfy (5.17) must be of size $\leq l^{O(1)}$, by a simple argument [BG08c, Prop. 8 and Lem. 3] based on the fact that the centralizer of a non-trivial element in a free group is cyclic: the centralizer is a free group (being a subgroup of a free group) but it cannot be of rank $\geq 2$, as it satisfies a non-trivial relation. Hence $\mu^{(2 k)}\left(G^{\prime \prime}\right)$, and thus $\mu^{(k)}\left(G^{\prime}\right)$, is indeed small:

$$
\mu^{(k)}\left(G^{\prime}\right) \ll \mu^{(2 k)}\left(G^{\prime \prime}\right) \ll \frac{(2 k)^{O(1)}}{r^{2 k}} \ll \frac{1}{p^{\eta}},
$$

where $\eta>0$ depends only on $c$, and thus only on $A$.

(For general $G$, showing that there is no concentration in a proper subgroup $G^{\prime}$ is a much more delicate matter. A fully general solution was given by GV12 ("escape in mass from subvarieties").)

Stage 2 (Decrease in $\mu^{(k)}$ for $k$ medium-sized, due to $\left|A^{3}\right| \geq|A|^{1+\delta}$ ). We are in the case in which one of the main results in this survey (Theorem 1.1) will be applied (via Proposition 3.5). Consider $\mu^{(k)}, \mu^{(2 k)}, \mu^{(4 k)}$, etc. At each step, we apply Proposition 3.5 (the flattening lemma) with $K=p^{\delta^{\prime}}, \delta^{\prime}>0$ to be set later. If (3.5) fails every time, we obtain $\left|\mu^{\left(2^{r} k\right)}\right|_{2}^{2}<1 /|G|$ after $r=O_{\delta^{\prime}, \eta}(1)$ steps; we then go to Stage 3.

Suppose (3.5) holds for some $k^{\prime}=2^{j} k, j \ll_{\delta^{\prime}, \eta} 1$. Then Proposition 3.5 gives that there is a $p^{O\left(\delta^{\prime}\right)}$-approximate subgroup $H \subset G$ of size $\ll p^{O\left(\delta^{\prime}\right)} /\left|\mu^{\left(k^{\prime}\right)}\right|_{2}^{2}$ and an element $g \in G$ such that $\mu^{\left(k^{\prime}\right)}(H g) \gg p^{-O\left(\delta^{\prime}\right)}$. In particular, $\left|H^{3}\right|<|H|^{1+O\left(\delta^{\prime} / \eta\right)}$. Choosing $\delta^{\prime}>0$ small enough, we get a contradiction to Theorem 1.1] unless $|H| \geq|G|^{1-O(\delta)}$ (where we can make $\delta$ as small as we want) or $H$ is contained in a proper subgroup $G^{\prime}$ of $G$.

If $|H| \geq|G|^{1-O(\delta)}$, then $|H| \ll p^{O\left(\delta^{\prime}\right)} /\left|\mu^{\left(k^{\prime}\right)}\right|_{2}^{2}$ implies that $\left|\mu^{\left(k^{\prime}\right)}\right|_{2}^{2} \ll 1 /|G|^{1-\delta-\delta^{\prime}}$, and we go to Stage 3. Assume, then, that $H$ is contained in a proper subgroup $G^{\prime}$ of $G$. Then $\mu^{\left(k^{\prime}\right)}\left(G^{\prime} g\right) \gg|G|^{-O\left(\delta^{\prime}\right)}$. This implies that $\mu^{(k)}\left(G^{\prime} g\right) \gg|G|^{-O\left(\delta^{\prime}\right)}$ ( simply because $\mu^{\left(k^{\prime}\right)}=\mu^{(k)} * \mu^{\left(k^{\prime}-k\right)}$ ), i.e., $\mu^{(k)}$ is concentrated in a subgroup, in contradiction (once we set $\delta^{\prime}$ small enough) with what we proved in Stage 2.

Stage 3 (Amplifying the effect of $\left|\mu^{(k)}\right|_{2}$ small by eigenvalue multiplicity). We have got to an $\ell=k^{\prime} \leq 2^{O_{\delta^{\prime}, \eta}(1)} k \ll_{A, \delta} \log p \ll \log |G|$ such that $\left|\mu^{(\ell)}\right|_{2}^{2} \ll 1 /|G|^{1-\delta}$, where $\delta>0$ is as small as we want. This is still weaker than a bound on $\ell^{2}$ mixing time (meaning an $\ell$ such that $\left.\left|\mu^{\ell}-1 /\right| G\right|_{2} ^{2} \ll \epsilon /|G|$ ), which is itself, in general, weaker than expansion. Let us see, however, how to get to expansion by using the high multiplicity of eigenvalues (\$5.3). (This is as in Sarnak and Xue SX91.) The trace of $\mathscr{A}^{2 \ell}$ is, on the one hand, $|G|\left|\mu^{\ell}\right|_{2}^{2}$ (by definition of trace, since the probability of $x$ returning to $x$ after $2 k$ steps of a random walk is $\left.\left|\mu^{\ell}\right|_{2}^{2}\right)$, and, on the other, $\sum \lambda_{i}^{2 \ell}$ (sum of eigenvalues). Hence

$$
m_{1} \lambda_{1}^{2 \ell} \leq \sum \lambda_{i}^{2 \ell}=|G|\left|\mu^{\ell}\right|_{2}^{2} \ll \frac{|G|}{|G|^{1-\delta}}=|G|^{\delta},
$$


where $m_{1}$ is the multiplicity of $\lambda_{1}$. As we saw in $55.3, m_{1}=(p-1) / 2 \gg|G|^{1 / 3}$. Thus, $\lambda_{1}^{2 \ell} \gg|G|^{\delta-1 / 3}$. We set $\delta<1 / 3(\delta=1 / 6$, say $)$ and obtain that $\lambda_{1} \leq 1-\epsilon$, where $\epsilon>0$ depends only on $A$ (and $\delta$, which is now fixed).

Applications have called for generalizing Theorem 1.3 in two directions. One is that of changing the Lie type. Here the first step was taken by Bourgain and Gamburd themselves BG08a]; a fully general statement for all perfect $G$ is due to Salehi-Golsefidy and Varjú GV12. (We have already discussed one of the main issues involved in a generalization, namely, avoiding concentration in subgroups.) The other kind of generalization consists in changing the ground ring. For many applications, the most important change turns out not to be changing $\mathbb{F}_{p}$ for $\mathbb{F}_{q}$, but changing $\mathbb{Z} / p \mathbb{Z}$ for $\mathbb{Z} / d \mathbb{Z}$. (This is needed for the the affine sieve [BGS10], one of the main ways in which results in the area get applied nowadays.) For $\mathrm{SL}_{2}$ and $d$ square-free, this was done in BGS10; Var12 and GV12 solved the problem for $G$ general and $d$ square-free; BG08b addressed $\mathrm{SL}_{2}$ and $d=p^{k}$, and BG09 did the same for $\mathrm{SL}_{n}$ and $d=p^{k}$. So far the only result for general moduli $d$ is [BV12, which treats $\mathrm{SL}_{n}$; as of the time of writing, the case of $G$ general and $d$ general is not yet finished.

\section{Growth in permutation groups}

6.1. Introduction. Our aim now will be to give some of the main ideas in the proof of quasipolynomial diameter for all Cayley graphs of the symmetric and alternating groups (Theorem 1.4):

$$
\operatorname{diam}(\Gamma(G, A)) \ll \exp \left((\log n)^{O(1)}\right)
$$

for any transitive group $G$ on $n$ elements and any $A \subset G$ generating $G$. (Here $O(1)$ is just $4+\epsilon$.)

The proof uses much of the foundational material we gave in 4 . The structure of the proof is very different from that for linear algebraic groups, however. In particular, we do not have access to dimensional bounds (since there is no clear meaning to "dimension" in a permutation group) or to escape-from-subvarieties arguments (essentially for the same reason).

Additional tools come from two sources. There were existing results on diameters of permutation groups: among those that were particularly useful, BS92, Thm. 1.1] reduces the problem to that for $\langle A\rangle=\operatorname{Alt}(n)$ (intuitively the hardest case) and BBS04 proves a polynomial diameter bound provided that $A$ contains at least one element other than the identity with small support. Note that BBS04 already uses the fact that even a very short random walk in $\operatorname{Sym}(n)$ takes an element of $\Omega=\{1,2, \ldots, n\}$ to any other element with almost uniform distribution.

Before [HS14], the strongest bound on the diameter of permutation groups was that of Babai and Seress [BS88, who showed that, for any permutation group $G$ on $n$ elements and any $A \subset G$ generating $G$,

$$
\operatorname{diam}(\Gamma(G, A)) \leq \exp ((1+o(1)) \sqrt{n \log n}) .
$$

While this is much weaker than the bound in Theorem [1.4 it does not assume transitivity (and indeed it can be tight for non-transitive groups). Moreover, the proof (see also BS87a and BLS87]) contains an idea that was useful in HS14, $\S 3.6]$ - namely, that prefixes can be played with to give the existence of non-identity elements with small support [BS88, Lem. 1]. 
Another key source of ideas came from existing work on Classification-free results on subgroups of $\operatorname{Sym}(n)$. The Classification of Finite Simple Groups is a result whose first proof spanned many volumes; its acceptance was gradual - even the date of its completion, at some point in the 1980s, is unclear. Thus, there was an interest in what Bab82] called "intelligible proofs of the asymptotic consequences of the Classification Theorem". Work in this direction includes Bab82, Pyb93, and [LP11].

Precisely because this work had combinatorial, relatively elementary bases, it turned out to be very robust: that is, these are results on the size of subgroups that can be generalized to any subsets that grow slowly under multiplication. (The basic idea here is as in the orbit-stabilizer theorem and its consequences (\$4.1): these are bounds on the size of a subgroup $H$ that are based on maps or processes that multiply $H$ by itself a few times ( $\leq k$ times, say); thus, if instead of having a subgroup $H$ we have a set $A$, we still have a result - just one where $H$ gets replaced sometimes by $A$ and sometimes by $A^{k}$, as we shall see.)

Just as a generalization of LP11 played an important role in both HW08 and BGT11, a generalization of Bab82 and Pyb93 plays an important role in HS14. What HS14] uses is not the final result in Bab82, but rather an intermediate result, the "splitting lemma". This is a result based on what is called the probabilistic method in combinatorics (generally, as in AS00, traced back to Erdös). This method is based on the observation that, if we show that something happens with positive probability, then it happens sometimes; thus, if we impose a convenient distribution (often the uniform one) on some initial objects, and we obtain that they then satisfy a certain property with positive probability, we have shown that a configuration of objects satisfying the property exists. The objects in Bab82] are elements of a group $H$. Now, we, in [HS14], do not have the right to choose elements of $H$ at random; to do so would be to assume what we are trying to prove, namely, a small bound on the diameter. Instead (as in BBS04]) we mimic the effects of a uniform distribution by means of a random walk; since the set $\{1,2, \ldots, n\}$ being acted upon is small, a short random walk is enough to give a distribution very close to uniform.

The proof in HS14 contains many other elements; a full outline is given in [HS14, §1.5]. Here, let us focus on a crucial part: the generalization of Babai's splitting lemma, and its application by means of the orbit-stabilizer theorem to create elements of $A^{k}$ in small subgroups of $\operatorname{Sym}(n)$. We will then look at a different part of the proof, giving a result of independent interest (Proposition 6.8) on small generating sets. This will demonstrate how different random processes - not just random walks on graphs - can be used to give explicit results on growth and generation.

6.1.1. Notation. As we said earlier, we will follow here the sort of notation that is current in the literature on permutation groups: actions $G \curvearrowright X$ are by default on the right, $x^{g}$ means $g(x)$ (so that $\left.x^{g h}=\left(x^{g}\right)^{h}\right), x^{A}$ is the orbit of $x$ under $A \subset G$, and $A_{x}$ means $\operatorname{Stab}(x) \cap A$. There are two different kind of stabilizers of a set $S \subset X$ : the setwise stabilizer

$$
A_{S}=\left\{g \in A: S^{g}=S\right\}
$$

and the pointwise stabilizer

$$
A_{(S)}=\left\{g \in A: x^{g}=x \quad \forall x \in S\right\} .
$$

(The notation here is as in DM96] and [Ser03, not as in Wie64.) 
6.2. Random walks and elements of small support. We will start with some basic material on random walks. We will then be able to go briefly over the proof of BBS04.

Let us start by defining our terms. We will work with a directed multigraph $\Gamma$, that is, a graph where the edges are directed (i.e., they are arrows) and may have multiplicity $>1$. (The setting we will work in now is more general than that of Cayley graphs.) We assume that $\Gamma$ is strongly connected (i.e., there is a path respecting the arrows between any two points in the vertex set $V(\Gamma)$ ), regular of valency (or degree) $d$ (meaning that there are $d$ arrows (counted with multiplicity) going out of every vertex in $V(\Gamma)$ ), and symmetric (i.e., the number of arrows from $x$ to $y$ is the same as the number of arrows from $y$ to $x$, counting with multiplicity in both cases).

We will study a lazy random walk: a particle moves from vertex to vertex, and at each point in time, if it is at a vertex $x$ and the arrows going out of $x$ end at the vertices $x_{1}, x_{2}, \ldots, x_{d}$ (with repetitions possible), the particle decides to be lazy (i.e., stays at $x$ ) with probability $1 / 2$, and moves to $x_{i}$ with probability $1 / 2 d$. (Studying a lazy random walk is a well-known trick used to avoid the possible effects of large negative eigenvalues of the adjacency matrix.)

Let $x, y \in V(\Gamma)$. We write $p_{k}(x, y)$ for the probability that a particle is at vertex $y$ after $k$ steps of a lazy random walk starting at $x$. For $\epsilon>0$ given, the $\left(\ell_{\infty}, \epsilon\right)$-mixing time is the least $k$ such that

$$
\left|p_{k}(x, y)-\frac{1}{|V(\Gamma)|}\right|_{\infty} \leq \frac{\epsilon}{|V(\Gamma)|} .
$$

(A mixing time is a time starting at which the outcome of a random walk is very close to a uniform distribution; the norm (e.g., $\ell_{\infty}$ ) and the tolerance (namely, $\epsilon$ ) have to be specified (as they are here) for "very close" to have a precise meaning.)

As before, the normalized adjacency matrix $\mathscr{A}$ is the operator taking a function $f: V(\Gamma) \rightarrow \mathbb{C}$ to the function $\mathscr{A} f: V(\Gamma) \rightarrow \mathbb{C}$ defined by

$$
\mathscr{A} f(x)=\frac{1}{d} \sum_{\text {arrows } v} f(\operatorname{wead}(v)) .
$$

(An arrow goes from its tail to its head.) Let $f_{0}, f_{1}, f_{2}, \ldots$ be a full set of eigenvectors corresponding to the eigenvalues $1=\lambda_{0} \geq \lambda_{1} \geq \lambda_{2} \geq \cdots$ of $\mathscr{A}$. If $\Gamma$ is regular and symmetric, then $\mathscr{A}$ is a symmetric operator, and so all $\lambda_{i}$ are real, and we can also assume all the $f_{i}$ to be real valued and orthogonal to each other. The following fact is well known.

Lemma 6.1. Let $\Gamma$ be a connected, regular, and symmetric multigraph of valency $d$ and with $N$ vertices. Then the $\left(\ell_{\infty}, \epsilon\right)$-mixing time is at most $N^{2} d \log (N / \epsilon)$.

The proof contains two steps: a trivial bottleneck bound gives a lower bound on the eigenvalue gap $\lambda_{0}-\lambda_{1}=1-\lambda_{1}$, and a lower bound on the eigenvalue gap gives an upper bound on the mixing time.

Proof. Let $f_{1}$ be an eigenvector corresponding to $\lambda_{1}$; since $f_{1}$ is orthogonal to the constant function $f_{0}$, the maximum $r_{+}$and the minimum $r_{-}$of $f_{1}(x)$ obey $r_{+}>$ $0>r_{-}$. By pigeonhole, there is an $r \in\left(r_{-}, r_{+}\right]$such that there are no $x \in V(\Gamma)$ with $f_{1}(x) \in(r-\eta, r)$, where $\eta=\left(r_{+}-r_{-}\right) / N$. Let $S=\left\{x \in V(\Gamma): f_{1}(x) \geq r\right\}$. Clearly, $S$ is neither empty nor equal to all of $V(\Gamma)$. 
Since $\Gamma$ is connected, there is at least one $x \in S$ with at least one arrow starting at $x$ and ending outside $S$. (This is the same as saying that the bottleneck of a connected graph is $\geq 1 / N d$.) Hence

$$
\sum_{x \in S} \mathscr{A} f_{1}(x) \leq-\frac{\eta}{d}+\sum_{x \in S} f_{1}(x)
$$

Again by $\left\langle f_{0}, f_{1}\right\rangle=0$, the average of $f_{1}(x)$ over $S$ is $>0$; trivially, it is also $<r_{+}$. Thus, 6.1) gives us

$$
\sum_{x \in S} \mathscr{A} f_{1}(x) \leq\left(1-\frac{1}{N|S| d}\right) \sum_{x \in S} f_{1}(x) .
$$

Therefore, $\lambda_{1} \leq 1-1 / N|S| d \leq 1-1 / N^{2} d$.

This implies the desired bound on the mixing time (exercise 10 ) by an idea already used in $\$ 5.3$, every step of the random walk multiplies the vector describing the probability distribution of the particle by $(\mathscr{A}+I) / 2$, and so anything orthogonal to a constant function gets multiplied by $\lambda_{1}=1-1 / N^{2} d$ (or less) repeatedly.

Lemma 6.1 may look weak, but it is actually quite useful for $N$ small, i.e., graphs with small vertex sets. When we work with a permutation group $G \leq \operatorname{Sym}(n)$, we may not have all the geometry we had at hand when working with linear algebraic groups, but we do have something else - an action on the small set $\{1,2, \ldots, n\}$ (and tuples thereof). That action gives rise to graphs with small vertex sets, allowing us to use Lemma 6.1.

First, we prove that we can mimic the uniform distribution on $k$-tuples by relatively short random walks. This is just as in BBS04, §2].

A $k$-transitive subgroup of $\operatorname{Sym}(n)$ is one whose action on the set of $k$-tuples of distinct elements of $\{1,2, \ldots, n\}$ is transitive.

Lemma 6.2. Let $G$ be a $k$-transitive subgroup of $\operatorname{Sym}(n)$. Let $A$ be a set of generators of $G$. Then there is a subset $A_{0} \subset A \cup A^{-1}$ such that the following holds.

For $\epsilon>0$ arbitrary, for any $\ell \geq 4 n^{2 k+1} \log \left(n^{k} / \epsilon\right)$ and for any $k$-tuples $\vec{x}, \vec{y}$ of distinct elements of $\{1,2, \ldots, n\}$, the probability that the outcome $g \in\left\langle A_{0}\right\rangle$ of a lazy random walk of length $\ell$ (on the graph $\Gamma\left(\left\langle A_{0}\right\rangle, A_{0}\right)$, starting at $e$ ) take $\vec{x}$ to $\vec{y}$ is at least $(1-\epsilon)(n-k) ! / n$ ! and at most $(1+\epsilon)(n-k) ! / n !$.

The number of $k$-tuples of distinct elements of $\{1,2, \ldots, n\}$ is, of course, $n ! /(n-k) !$.

Proof. Since $A$ may be large and it will be best to work with a generating set that is not very large, we start by choosing a subset of $A$ that still generates $G$. This we do simply by choosing an element $g_{1} \in A$, and then an element $g_{2} \in A$ such that $\left\langle g_{1}\right\rangle \lesseqgtr\left\langle g_{1}, g_{2}\right\rangle$, and then a $g_{3} \in A$ such that $\left\langle g_{1}, g_{2}\right\rangle \lesseqgtr\left\langle g_{1}, g_{2}, g_{3}\right\rangle$, etc., until we get $\left\langle g_{1}, g_{2}, \ldots, g_{r}\right\rangle=\langle A\rangle=G(r \geq 1)$. Since the longest subgroup chain in $\operatorname{Sym}(n)$ is of length $\leq 2 n-3$ Bab86, 11 we see that $r \leq 2 n-2<2 n$. Let $A_{0}=\left\{g_{1}, g_{1}^{-1}, \ldots, g_{r}, g_{r}^{-1}\right\}$.

\footnotetext{
${ }^{10}$ See the proof of [HS14, Lem. 4.1] (or any of many other sources, e.g., Lov96, Thm. 5.1]) for a solution. It is easy to do this suboptimally and obtain an extra factor of $\left|p_{k}(x, y)-1 / N\right| \leq N \lambda_{2}^{k}$ instead of $\left|p_{k}(x, y)-1 / N\right| \leq \lambda_{2}^{k}$.

${ }^{11}$ The trivial bound is $(\log n !) / \log 2$ : in a subgroup chain $H_{1} \lesseqgtr H_{2} \lesseqgtr \cdots \lesseqgtr H_{k}$, we have $\left|H_{2}\right| \geq 2\left|H_{1}\right|,\left|H_{3}\right| \geq 2\left|H_{2}\right|$, etc., simply because the index of a proper subgroup of a group is always $\leq 2$.
} 
Now define the multigraph $\Gamma$ by letting the set of vertices consist of all $k$-tuples of distinct elements of $\{1,2, \ldots, n\}$. Draw an arrow between $\vec{z}=\left(z_{1}, z_{2}, \ldots, z_{k}\right)$ and $\left(z_{1}^{a}, z_{2}^{a}, \ldots, z_{k}^{a}\right)$ for every vertex (i.e., $k$-tuple) $\vec{z}$ and every $a \in A_{0}$. (This is, of course, a Schreier graph.) Finish by applying Lemma 6.1.

We will now see how to adapt the probabilistic method using Lemma 6.2 to approximate a uniform distribution by a short random walk.

Proposition 6.3 ([BBS04] $)$. Let $A \subset \operatorname{Sym}(n)$ generate a 3-transitive subgroup of $\operatorname{Sym}(n)$. Let $g \in A^{\ell_{0}}, \ell_{0} \geq 1$ arbitrary. Assume that $0<|\operatorname{supp}(g)|<n$. Then, for any $\epsilon>0$, there is an element $g^{\prime} \in\left(A \cup A^{-1}\right)^{\ell+4 \ell_{0}}, \ell \ll n^{7} \log (n / \epsilon), g \neq e$, such that

$$
\operatorname{supp}\left(g^{\prime}\right) \leq 3+3(1+O(\epsilon))|\operatorname{supp}(g)|^{2} / n,
$$

where the implied constant is absolute.

The conclusion is non-trivial only when $\operatorname{supp}(g)<n / 3$.

Proof. Given $\sigma \in \operatorname{Sym}(n)$ and $x \in\{1,2, \ldots, n\}$, let $h=\sigma^{-1} g \sigma$; thus, $\operatorname{supp}(h)=$ $(\operatorname{supp}(g))^{\sigma}$. Write $S$ for $\operatorname{supp}(g)$. When is $x$ in the support of the commutator $[g, h]=g^{-1} h^{-1} g h$ ? (Defining the commutator in this way, rather than as $g h g^{-1} h^{-1}$, is standard in the study of permutation groups.) There are three possibilities:

(a) $x \in S$ and $x^{g^{-1}} \in \operatorname{supp}(h)$, i.e., $x^{g^{-1}} \in S \cap S^{\sigma}$;

(b) $x \in S, x^{g^{-1}} \notin \operatorname{supp}(h)$ and $x \in \operatorname{supp}(h)$, and so, in particular, $x \in S \cap S^{\sigma}$;

(c) $x \notin S, x \in \operatorname{supp}(h)$ and $x^{h^{-1}} \in S$, and so, in particular, $x^{h^{-1}} \in S \cap S^{\sigma}$.

Thus, $\operatorname{supp}([g, h])$ is contained in

$$
\left(S \cap S^{\sigma}\right) \cup\left(S \cap S^{\sigma}\right)^{g} \cup\left(S \cap S^{\sigma}\right)^{h} .
$$

Now let $\sigma \in\left(A \cup A^{-1}\right)^{\ell^{\prime}}$ be the outcome of a lazy random walk of length $\ell^{\prime}=$ $\left\lceil 4 n^{2 k+1} \log \left(n^{k} / \epsilon\right)\right\rceil, k=3$. Lemma 6.2 tells us that, for any $x, x^{\prime} \in\{1,2, \ldots, n\}, \sigma$ will take $x$ to $x^{\prime}$ with probability between $(1-\epsilon) / n$ and $(1+\epsilon) / n$. Since expectation is additive, it follows that, for every $S \subset\{1,2, \ldots, n\}$,

$$
\begin{aligned}
\mathbb{E}\left(\left|S \cap S^{\sigma}\right|\right) & =\sum_{x^{\prime} \in S} \operatorname{Prob}\left(x^{\prime} \in S^{\sigma}\right)=\sum_{x^{\prime} \in S} \sum_{x \in S} \operatorname{Prob}\left(x^{\sigma}=x^{\prime}\right) \\
& =\sum_{x^{\prime} \in S} \sum_{x \in S} \frac{1+O(\epsilon)}{n}=(1+O(\epsilon)) \frac{|S|^{2}}{n} .
\end{aligned}
$$

Writing $S=S$, we see that

$$
\begin{aligned}
\mathbb{E}(|\operatorname{supp}([g, h])|) & \leq \mathbb{E}\left(\left|S \cap S^{\sigma}\right|\right)+\mathbb{E}\left(\left|\left(S \cap S^{\sigma}\right)^{g}\right|\right)+\mathbb{E}\left(\left|\left(S \cap S^{\sigma}\right)^{h}\right|\right) \\
& =3 \cdot \mathbb{E}\left(\left|S \cap S^{\sigma}\right|\right) \leq 3(1+O(\epsilon)) \frac{|S|^{2}}{n}=3(1+O(\epsilon)) \frac{|S|^{2}}{n} .
\end{aligned}
$$

Now we could conclude that there exists a $\sigma \in\left(A \cup A^{-1}\right)^{\ell^{\prime}}$ such that $|\operatorname{supp}([g, h])|$ is at most $3(1+O(\epsilon))|S|^{2} / n$. We forgot to take care of one detail, however: $\left[g, \sigma^{-1} g \sigma\right]$ could be the identity. Fortunately $\ell^{\prime}$ is large enough that Lemma 6.2 assures us that, even if we specify that $y_{i}^{\sigma}=y_{i}^{\prime}$ for some $y_{i}, y_{i}^{\prime} \in\{1,2, \ldots, n\}$ $\left(i=1,2 ; y_{1} \neq y_{2}, y_{1}^{\prime} \neq y_{2}^{\prime}\right)$, the probability that $x^{\sigma}=x^{\prime}$ for $x, x^{\prime} \in\{1,2, \ldots, n\}$ $\left(x \neq y_{i}, x^{\prime} \neq y_{i}^{\prime}\right.$ for $\left.i=1,2\right)$ is $(1+O(\epsilon)) /(n-2)=(1+O(\epsilon)) / n$. (This is why we let $k=3$ and not $k=1$.) We choose $y_{1} \in S, y_{1}^{\prime} \in S, y_{2} \notin S, y_{2}^{\prime}=\left(y_{1}^{\prime}\right)^{g^{-1}}$. Then (by a brief computation) $\left(y_{1}^{\prime}\right)^{[g, h]} \neq y_{1}^{\prime}$, and so $[g, h]$ is not the identity. 
The analysis goes on as before, except we obtain

$$
\mathbb{E}\left(\left|\left\{x \in S \cap S^{\sigma}\right\}\right|\right) \leq 1+(1+O(\epsilon)) \frac{|S|^{2}}{n}
$$

(or $2+(1+O(\epsilon))|S|^{2} / n$ for general $S$; we are using the fact that $y_{2}$ and $y_{2}^{\prime}$ are never both in $S$ in our case) and so

$$
\mathbb{E}(|\operatorname{supp}([g, h])|) \leq 3+3(1+O(\epsilon)) \frac{|S|^{2}}{n} .
$$

Thus, there is a $\sigma \in\left(A \cup A^{-1}\right)^{\ell^{\prime}}$ such that $g^{\prime}=[g, h]=\left[g, \sigma^{-1} g \sigma\right]$ has support $\leq 3+3(1+O(\epsilon))|S|^{2} / n$.

Corollary 6.4 ([BBS04] $)$. Let $A \subset \operatorname{Sym}(n)\left(A=A^{-1}\right)$ generate a 3-transitive subgroup $G$ of $\operatorname{Sym}(n)$. Assume there is a $g \in A, g \neq e$, with $|\operatorname{supp}(g)| \leq(1 / 3-\epsilon) n$, $\epsilon>0$. Then

$$
\operatorname{diam}(\Gamma(G, A)) \ll_{\epsilon} n^{c_{1}}(\log n)^{c_{2}},
$$

where $c_{1}=8$ and $c_{2}$ is absolute.

Proof. Apply Proposition 6.3, then apply it again with $g^{\prime}$ instead of $g$, and again and again. After $O(\log \log n)$ steps, we will have obtained an element $g_{1} \in A^{\ell_{1}}$, $\ell_{1} \ll_{\epsilon} n^{7}(\log n)^{c}$, such that $g_{1} \neq e$ and $\left|\operatorname{supp}\left(g_{1}\right)\right| \leq 3$. A brief argument suffices to show that $A^{n^{3}}$ acts 3 -transitively (i.e., for any two 3-tuples of distinct elements of $\{1,2 \ldots, n\}$, there is an element of $A$ taking one to the other). Hence either all 2 -cycles or all 3 -cycles are in $A^{\ell_{1}+2 n^{3}}$ (in that they can be obtained by conjugating $g_{1}$ by elements of $A^{n^{3}}$ ). If at least one element $h$ of $A$ is not in $\operatorname{Alt}(n)$, it is easy to construct a 2-cycle (and hence all 2-cycles) by using $h$ and some well-chosen 3 -cycles. We then construct every element of $\langle A\rangle$ (which, without having meant to, we are now showing to be either $\operatorname{Alt}(n)$ or $\operatorname{Sym}(n))$ as a product of length at most $n$ in our 2-cycles (if $A \not \subset \operatorname{Alt}(n)$ ) or 3-cycles (if $A \subset \operatorname{Alt}(n)$ ).

There is clearly some double-counting going on in the proof of Proposition 6.3 , A more careful counting argument gives an improved statement that results in a version of Corollary 6.4 with $1 / 2$ instead of $1 / 3$. Redoing Proposition 6.3 with well-chosen words other than $[g, h]$ results in still better bounds; $\mathrm{BGH}^{+} 14$ gives $\operatorname{diam}(\Gamma(G, A)) \ll n^{O(1)}$ provided that there is a $g \in A, g \neq e$ with $|\operatorname{supp}(g)| \leq$ $0.63 n$.

The moral of this section is that short random walks can be enough for "the probabilistic method" in combinatorics (showing existence by showing positive probability) to work, in that they serve to approximate the uniform distribution on $k$-tuples $(k$ small) very well.

6.3. Large orbits, pointwise stabilizers, and stabilizer chains. The following result was a key part of the proof of Babai's elementary bound on the size of 2transitive permutation groups other than $\operatorname{Alt}(n)$ and $\operatorname{Sym}(n)$ Bab82. It has a probabilistic proof.

Lemma 6.5 (Babai's splitting lemma Bab82]). Let $H \leq \operatorname{Sym}(n)$ be 2-transitive. Let $\Sigma \subset\{1,2, \ldots, n\}$. Let $\rho>0$. Assume that there are at least $\rho n(n-1)$ ordered pairs $(\alpha, \beta)$ of distinct elements of $\{1,2, \ldots, n\}$ such that there is no $g \in H_{(\Sigma)}$ with $\alpha^{g}=\beta$. Then there is a subset $S$ of $H$ with

$$
H_{\left(\Sigma^{S}\right)}=\{e\}
$$

and $|S| \ll_{\rho} \log n$. 
Proof. Let $\alpha, \beta$ be distinct elements of $\{1,2, \ldots, n\}$. Let $h \in H$. Suppose there is a $g^{\prime} \in H_{\left(\Sigma^{h}\right)}$ such that $\alpha^{g^{\prime}}=\beta$. Then $g=h g^{\prime} h^{-1}$ is an element of $H_{(\Sigma)}$ taking $\alpha^{h^{-1}}$ to $\beta^{h^{-1}}$.

The elements $h$ of $H$ such that $h^{-1}$ takes $(\alpha, \beta)$ to a given pair $\left(\alpha^{\prime}, \beta^{\prime}\right)$ of distinct elements of $\{1,2, \ldots, n\}$ form a coset of $H_{(\alpha, \beta)}$. Hence, if we choose an element $h \in H$ at random, $h^{-1}$ is equally likely to take $(\alpha, \beta)$ to any given pair $\left(\alpha^{\prime}, \beta^{\prime}\right)$. In particular, the probability that it will take $(\alpha, \beta)$ to a pair $\left(\alpha^{\prime}, \beta^{\prime}\right)$ such that there is no $g \in H_{(\Sigma)}$ taking $\alpha$ to $\beta$ is at least $\rho$. By what we were saying, this would imply that there is no $g^{\prime} \in H_{\left(\Sigma^{h}\right)}$ such that $\alpha^{g^{\prime}}=\beta$.

Now take a set $S$ of $r$ elements of $H$ taken uniformly at random. By the above, for a given $(\alpha, \beta)$, the probability that, for every $h \in H$, there is a $g^{\prime} \in H_{\left(\Sigma^{h}\right)}$ such that $\alpha^{g^{\prime}}=\beta$ is at most $(1-\rho)^{r}$. There would be such a $g^{\prime}$ for every $h \in H$ if there were a $g^{\prime} \in H_{\Sigma^{S}}$ such that $\alpha^{g^{\prime}}=\beta$ (since such a $g^{\prime}$ would be good for every $h \in H)$. Hence, the probability that there is at least one pair $(\alpha, \beta)$ of distinct elements such that there is a $g^{\prime} \in H_{\Sigma S}$ with $\alpha^{g^{\prime}}=\beta$ is at most $n^{2}(1-\rho)^{r}$. For any $r>2(\log n) / \log (1 /(1-\rho))$, we get $n^{2}(1-\rho)^{r}<1$, and thus there exists a set $S$ of at most $r$ elements such that there is no such pair $(\alpha, \beta)$. If there is no such pair, then the only possible element of $H_{\Sigma^{S}}$ is the identity, i.e., $H_{\Sigma^{S}}=\{e\}$.

We wish to adapt Lemma 6.5 to hold for subsets $A \subset G$ instead of subgroups $H$. Here one of our leitmotifs reappears, but undergoes a change. Adapting a result on subgroups to hold for subsets is a recurrent idea that we have seen throughout this survey. However, so far, we have usually done this by relaxing the condition that $A$ be a subgroup into the condition that $A^{3}$ not be much larger than $A$. This is a tactic that often works when the underlying idea is basically quantitative, as is the case, e.g., for the orbit-stabilizer theorem.

Another tactic consists in redoing an essentially constructive proof keeping track of how many products are taken; this is, for example, how a lemma of Bochert's gets adapted in [HS14, Lem. 3.12]. To generalize Babai's splitting lemma, however, we will follow a third tactic - namely, making a probabilistic proof into what we can call a stochastic one, viz., one based on random walks, or, more generally, on a random process.

We already saw how to use random walks in this way in 6.2 (Babai, Beals, and Seress); the idea is to approximate the uniform distribution by a short random walk, using Lemma 6.2

Lemma 6.6 (Splitting lemma for sets ([HS14], Proposition 5.2)). Let $A \subseteq \operatorname{Sym}(n)$ with $A=A^{-1}, e \in A$, and $\langle A\rangle$ 2-transitive. Let $\Sigma \subseteq\{1,2, \ldots, n\}$. Let $\rho>0$. Assume that there are at least $\rho n(n-1)$ ordered pairs $(\alpha, \beta)$ of distinct elements of $\{1,2, \ldots, n\}$ such that there is no $g \in\left(A^{\left\lfloor 9 n^{6} \log n\right\rfloor}\right)_{(\Sigma)}$ with $\alpha^{g}=\beta$. Then there is a subset $S$ of $A^{\left\lfloor 5 n^{6} \log n\right\rfloor}$ with

$$
\left(A A^{-1}\right)_{\left(\Sigma^{S}\right)}=\{e\}
$$

and $|S| \ll_{\rho} \log n$.

Passing from the statement of Lemma 6.5 to the statement of Lemma 6.6 some instances of $H$ get replaced by $A$ and some get replaced by $A^{k}, k$ moderate. This is what makes it possible to give a statement that is true for general sets $A$ without 
assumptions on the size of $A^{3}$; of course, a moment's thought shows that the statement is particularly strong when $A$ grows slowly. This is much as in Proposition 5.2 or Proposition 5.3 .

Sketch of proof. Exercise. Adapt the proof of Lemma 6.5, using Proposition 6.3, It is useful to note that $g A_{\left(\Sigma^{g}\right)} g^{-1}=\left(g A g^{-1}\right)_{(\Sigma)}$.

Let us see how to use Lemma 6.6. (This part of the argument is similar to that in Pyb93, which sharpened the bounds in Bab82.) By pigeonhole, $\left(A A^{-1}\right)_{\Sigma^{S}}=\{e\}$ can be the case only if $|A| \leq n^{\left|\Sigma^{S}\right|}$, and that can happen only if

$$
|\Sigma| \gg \frac{\log |A|}{(\log n)^{2}}
$$

This means that there is a constant $c$ such that, for every $\Sigma \subset\{1,2, \ldots, n\}$ with $|\Sigma| \leq c(\log |A|) /(\log n)^{2}$, the assumption ("there are at least $\rho n(n-1)$ ordered pairs...") in Lemma 6.6 does not hold (since the conclusion cannot hold.)

Thus, for any $\sigma<1$, we are guaranteed to be able to find $\Sigma=\left\{\alpha_{1}, \alpha_{2}, \ldots, \alpha_{m}\right\}$, $m \gg_{\sigma}(\log |A|) /(\log n)^{2}$, such that, for $A^{\prime}=A^{\left\lfloor 9 n^{6} \log n\right\rfloor}$,

$$
\left|\alpha_{i}^{A_{\left(\alpha_{1}, \ldots, \alpha_{i-1}\right)}^{\prime}}\right| \geq \sigma n
$$

for every $1 \leq i \leq m$; we are setting $\rho=1-\sigma$. (The use of stabilizer chains $A>A_{\left(\alpha_{1}\right)}>A_{\left(\alpha_{1}, \alpha_{2}\right)}>\cdots$ goes back to the algorithmic work of Sims [Sim70], [Sim71, as does the use of the size of the orbits in (6.3); see [Ser03, §4.1].)

By (6.3), $\left(A^{\prime}\right)^{m}$ occupies at least $(\sigma n)^{m}$ cosets of the pointwise stabilizer $\operatorname{Sym}(n)_{(\Sigma)}$ (exercise; HS14, Lem. 3.17]), out of $n ! /(n-m) !<n^{m}$ possible cosets of $\operatorname{Sym}(n)_{(\Sigma)}$ in $\operatorname{Sym}(n)$. The number of cosets of $\operatorname{Sym}(n)_{(\Sigma)}$ in the setwise stabilizer $\operatorname{Sym}(n)_{\Sigma}$ is $m$ !, which is much larger than $n^{m} /(\sigma n)^{m}=(1 / \sigma)^{m}$. (We can work with $\sigma=9 / 10$, say.) A hybrid of Lemma 4.3 and Lemma 4.4 ([HS14, Lem. 3.7]) then shows immediately that $\left(A^{\prime}\right)^{2 m} \cap \operatorname{Sym}(n)_{\Sigma}$ intersects many $\left(\geq \sigma^{m} m !\right)$ cosets of $\operatorname{Sym}(n)_{(\Sigma)}$ (and, in particular, $\left.\left|\left(A^{\prime}\right)^{2 m} \cap \operatorname{Sym}(n)_{\Sigma}\right| \geq \sigma^{m} m !\right)$.

Let us see what we have got. We have constructed many elements of $\left(A^{\prime}\right)^{2 m} \subset$ $A^{n^{O(1)}}$ lying in a "special subgroup" $(\operatorname{Sym}(n))_{\Sigma}$. This is in loose analogy to the situation over linear algebraic groups, where we constructed many elements of $A^{2}$ lying in a special subgroup $T=C(g)$ (Corollary 5.4). Moreover, the elements of $\left(A^{\prime}\right)^{2 m} \cap(\operatorname{Sym}(n))_{\Sigma}$ will act (by conjugation) on an even more specia 12 subgroup, namely, $(\operatorname{Sym}(n))_{(\Sigma)}$.

This is a turning point in the proof of Theorem 1.4, just as Corollary 5.4 (or its weaker version, [Hel08, Prop. 4.1]) was a turning point in the proof of Theorem 1.1.

6.4. Constructing small generating sets. Let $A$ be a set of generators of $\operatorname{Sym}(n)$ or $\operatorname{Alt}(n)$. The set $A$ may be large -inconveniently so for some purposes. Can we find a set $S \subset A^{\ell}$ of bounded size ( $\ell$ moderate) so that $S$ generates $\langle A\rangle$ ?

\footnotetext{
${ }^{12}$ Note the shift, or non-shift, in the meaning of "special" (dictated by the requirements of the problems at hand). Before, a special subgroup was exactly that, namely, an algebraic subgroup ("special" meaning "lying on a set of positive codimension, algebraically speaking"). Here the role of "special subgroups" is played by stabilizers (in relation to the natural action of $\operatorname{Sym}(n)$ on $\{1,2, \ldots, n\}$, or powers thereof). The difference is, however, less than it seems at first: the algebraic subgroup $T$ is also given as a stabilizer $C(g)$ (in relation to the action by conjugation of $G$ on itself, which is a natural action of $G$ on an affine space.)
} 
This is a question that arises in the course of the proof of Theorem 1.4. Addressing it will give us the opportunity to show how to use stochastic processes other than a simple random walk in order to put a generalized probabilistic method into practice.

Let us start with a simple lemma.

Lemma 6.7 ([S14, Lem. 4.3]). Let $A \subset \operatorname{Sym}(n), e \in A$. Assume $\langle A\rangle$ is transitive. Then there is a $g \in A^{n}$ such that $|\operatorname{supp}(g)| \geq n / 2$.

Sketch of proof. For every $i \in\{1,2, \ldots, n\}$, there is a $g_{i} \in A$ such that $i \in \operatorname{supp}(g)$ (why?). Consider $g=g_{1}^{r_{1}} g_{2}^{r_{2}} \cdots g_{n}^{r_{n}}$, where $r_{1}, r_{2}, \ldots, r_{n} \in\{0,1\}$ are independent random variables taking the values 0 and 1 with equal probability. Show that the expected value of $|\operatorname{supp}(g)|$ is at least $n / 2$ (exercise).

We will be using $g$ to move an element of $\{1,2, \ldots, n\}$ around and another element $h$ (produced by a random walk) in order to scramble $\{1,2, \ldots, n\}$.

Proposition 6.8 ([HS14, Lem. 4.5 and Prop. 4.6]). Let $A \subseteq \operatorname{Sym}(n)$ with $A=$ $A^{-1}$, let $e \in A$, and let $\langle A\rangle=\operatorname{Sym}(n)$ or $\operatorname{Alt}(n)$. Then there are $g \in A^{n}$ and $j, h \in A^{n^{O(\log n)}}$ such that $\langle g, j, h\rangle$ is transitive.

Extended sketch of proof. By Lemma 6.7, there is a $g \in A^{n}$ with $|\operatorname{supp}(g)| \geq n / 2$. Let $h \in A^{\ell}, \ell=n^{7 k}, k=\lceil 8 \log n\rceil$ (say) be the outcome of a lazy random walk on $\Gamma(G, A)$ of length $\ell$ (starting at $e$ ). We can assume $n$ is larger than an absolute constant.

We will consider words of the form

$$
f(\vec{a})=h g^{a_{1}} h g^{a_{2}} \cdots h g^{a_{k}},
$$

where $a_{1}, \ldots, a_{k} \in\{0,1\}$. We wish to show that, for $\beta \in\{1,2, \ldots, n\}$ taken at random (with the uniform distribution on $\{1,2, \ldots, n\}$ ), the orbit $\beta^{f(\vec{a})}, \vec{a} \in\{0,1\}^{k}$, is likely to be very large $\left(\gg n /(\log n)^{2}\right)$.

A simple sphere-packing bound shows that there is a set $V \subset\{0,1\}^{k},|V| \geq n$, such that the Hamming distance 13 between any two distinct elements of $V$ is at least $k / 5>\log _{2} n$ (exercise). We wish to show that, for $\beta$ random and $\vec{a}, \vec{a}^{\prime} \in V$ distinct, it is very unlikely that $\beta^{f(\vec{a})}$ equal $\beta^{f\left(\vec{a}^{\prime}\right)}$.

Write $\vec{a}=\left(a_{1}, a_{2}, \ldots, a_{k}\right), \vec{a}^{\prime}=\left(a_{1}^{\prime}, a_{2}^{\prime}, \ldots, a_{k}^{\prime}\right)$. Consider the sequences

$$
\begin{array}{r}
\beta_{0}=\beta, \beta_{1}=\beta_{0}^{h g^{a_{1}}}, \beta_{2}=\beta_{1}^{h g^{a_{2}}}, \ldots, \beta_{k}=\beta_{k-1}^{h g^{a_{k}}}, \\
\beta_{0}^{\prime}=\beta, \beta_{1}^{\prime}=\beta^{h g^{a_{1}^{\prime}}}, \beta_{2}^{\prime}=\left(\beta_{1}^{\prime}\right)^{h g^{a_{2}^{\prime}}}, \ldots, \beta_{k}^{\prime}=\left(\beta_{k-1}^{\prime}\right)^{h g^{a_{k}^{\prime}}} .
\end{array}
$$

It is very unlikely that $\beta^{h}=\beta$ (probability $\sim 1 / n$ ) or that $\beta^{h g}=\beta$, i.e., $\beta^{h}=\beta^{g^{-1}}$ (probability $\sim 1 / n$ ). If neither of these unlikely occurrences takes place, it is also very unlikely (total probability $\leq(1+o(1)) 4 / n$ ) that $\beta_{1}^{h}$ or $\beta_{1}^{h g}$ equal $\beta$ or $\beta^{h}$. The reason is that, since $\beta_{1}$ has not been seen "before" (i.e., $\left(\beta, \beta_{1}\right)$ is a pair of distinct elements), the distribution of $h^{\beta_{1}}$ is almost uniform, even conditionally on $\beta=x$, for any $x$. (This can be easily made rigorous; it is much as (for instance) in the proof of Proposition 6.3, right after (6.2).) Thus, the probability that $\beta_{1}^{h}=\beta$ (for example) is $\sim 1 / n$ (the same as the probability of $\beta_{1}^{h}=x$ for any $x$ other than $\beta^{h}$ ). Proceeding in this way, we obtain that it is almost certain (probability

${ }^{13}$ The Hamming distance $d(\vec{x}, \vec{y})$ between two elements $\vec{x}, \vec{y} \in\{0,1\}^{k}$ is the number of indices $1 \leq i \leq k$ such that $x_{i} \neq y_{i}$. 
$1-O(k / n))$ that $\beta_{1}, \beta_{2}, \ldots, \beta_{k}$ are all distinct. (Recall that, by Lemma 6.2 a random walk of length $\ell$ mixes $k$-tuples (and even $2 k$-tuples) of distinct elements. It is also relevant that $k$ is very small compared to $n$, as this means that hitting one of $k$ (or rather $2 k$ ) visited elements by picking an element of $\{1,2, \ldots, n\}$ at random is highly unlikely. We can keep our independence from the past as long as we do not go back to it.)

Let us see what happens to $\beta_{0}^{\prime}, \beta_{1}^{\prime}, \ldots$ in the meantime. Start at $i=0$ and increase $i$ by 1 repeatedly. As long as $a_{i}=a_{i}^{\prime}$, we have $\beta_{i}=\beta_{i}^{\prime}$. As soon as $a_{i} \neq a_{i}^{\prime}$ (denote by $i_{1}$ the first index $i$ for which this happens), we may have $\beta_{i} \neq \beta_{i}^{\prime}$; this happens when $\beta_{i-1}^{h} \in \operatorname{supp}(g)$, i.e., it happens with probability $\sim|\operatorname{supp}(g)| / n$. If this happens, then, by the same argument as above, it is highly likely that the two paths in (6.4) diverge, i.e., $\beta_{j} \neq \beta_{j}^{\prime}$ for all $j>i$, and, for that matter, that they also avoid each other's past $\left(\beta_{j} \neq \beta_{l}^{\prime}\right.$ for all $j>i$ and all $l<j$ ). (It is useful to keep track of the latter condition for the same reason as above, namely, to keep our independence from events that have already been determined.)

Since $\vec{a}, \vec{a}^{\prime}$ are at Hamming distance at least $n$ from each other, it is very unlikely that $\beta_{i-1}^{h} \in \operatorname{supp}(g)$ for all $i$ such that $a_{i} \neq a_{i}^{\prime}\left(\operatorname{probability} \leq(1-|\operatorname{supp}(g)| / n)^{n} \leq\right.$ $2^{-n}$, since there are $n$ such indices $i$ ). Hence the two paths almost certainly diverge - never to meet again, as we just showed; in particular, $\beta^{f(\vec{a})}$ and $\beta^{f\left(\vec{a}^{\prime}\right)}$ are almost certainly distinct. They are distinct with probability $\geq 1-O\left((\log n)^{2} / n\right)$ for any distinct $\vec{a}, \overrightarrow{a^{\prime}} \in V$ and $\beta \in\{1,2, \ldots, n\}$ random, to be precise.

By Cauchy-Schwarz, this implies that the expected value of $1 /\left|\beta^{\langle g, h\rangle}\right|$ for $\beta$ random is $O\left((\log n)^{2} / n\right)$. This implies, in turn, that the expected value of the number of orbits of $\langle g, h\rangle$ is $O\left((\log n)^{2}\right)$ (exercises).

A third element $j \in\langle A\rangle$ obtained by a random walk of length $\ell$ almost certainly merges these orbits, i.e., $\langle g, h, j\rangle$ is transitive. (Longer but easy exercise.) Hence there exist (many) $g, h, j \in A^{\ell}$ such that $\langle g, h, j\rangle$ is transitive.

Corollary 6.9 ([ $\mathrm{HS} 14$, Cor. 4.7]). Let $A \subseteq \operatorname{Sym}(n)$ with $A=A^{-1}$, let $e \in A$, and let $\langle A\rangle=\operatorname{Sym}(n)$ or $\operatorname{Alt}(n)$. Then, for every $k \geq 1$, there is a set $S \in A^{(3 n)^{k} n^{O(\log n)}}$ of size at most $3 k$ such that $\langle S\rangle$ is transitive.

In particular, if we want $\langle S\rangle$ to be $\operatorname{Sym}(n)$ or $\operatorname{Alt}(n)$ (something we do not need in the application in [HS14), then it is enough to set $k=6$, as the Classification of Finite Simple groups implies that a 6 -transitive group must be either $\operatorname{Sym}(n)$ or $\operatorname{Alt}(n)$.

Sketch of proof. Apply Proposition6.8 repeatedly, using Schreier generators to pass to pointwise stabilizers of $\{1\},\{1,2\}$, etc.

How far can arguments such as those in the proof of Proposition 6.8 be pushed? Here there is again a "classical" argument to be examined in the light of random processes and random walks, namely, the work of Broder and Shamir on the spectral gap of random graphs BS87b. The ideas there and those in Proposition 6.8 are some of the elements leading to [HSZ, which gives a bound of $O\left(n^{2}(\log n)^{O(1)}\right)$ on the diameter of $\Gamma(\langle A\rangle, A)$ for $A=\{g, h\}, g, h \in \operatorname{Sym}(n)$ random.

6.5. The action of the setwise stabilizer on the pointwise stabilizer. How is this all put together to give Theorem 1.4? The entire argument is outlined in detail in [HS14, §1.5]. Here, let us go over a crucial step and look quickly at what then follows, skipping some of the complications. 
We are working with a set $A \subset \operatorname{Sym}(n)$ generating $\operatorname{Alt}(n)$ or $\operatorname{Sym}(n)$. By the end of 6.3 , we had constructed a large subset $\Sigma \subset\{1,2, \ldots, n\}(m=|\Sigma| \gg$ $\left.(\log |A|) /(\log n)^{2}\right)$ such that $\left(A^{\prime \prime}\right)_{\Sigma}\left(\right.$ where $\left.A^{\prime \prime}=\left(A^{\prime}\right)^{2 m}=A^{n^{O(1)}}\right)$ intersects $\geq$ $\sigma^{m} m$ ! cosets of $\operatorname{Sym}(n)_{(\Sigma)}$; in other words, the projection of $\left(A^{\prime \prime}\right)_{\Sigma}$ to $\operatorname{Sym}(\Sigma)$ (by restriction to $\Sigma$ ) has $\geq \sigma^{m} m$ ! elements.

By the trick of demanding (6.3) for $m+1$ rather than $m$, we can ensure that $\left\langle\left(A^{\prime \prime}\right)_{(\Sigma)}\right\rangle$ has at least one large orbit $\Gamma(|\Gamma| \geq \sigma n)$. We can actually assume that $\left\langle\left(A^{\prime \prime}\right)_{(\Sigma)}\right\rangle$ acts as Sym or Alt on $\Gamma$, since otherwise we are done by a different argument (called descent in [HS14, §6], as in "infinite descent", because it is inductive; it is also the one step that involves the Classification of Finite Simple Groups). Then, by Corollary 6.9 there is a set $S \subset\left(\left(A^{\prime \prime}\right)^{n^{O(\log n)}}\right)_{(\Sigma)},|S| \leq 6$, such that $\langle S\rangle$ acts as a 2-transitive group on $\Gamma$.

We now consider the action of the elements of $\left(A^{\prime \prime}\right)_{\Sigma}$ on the elements of $S$ by conjugation. By the orbit-stabilizer principle (Lemma 4.1), either (a) there is an element $g \neq e$ of $\left(A^{\prime \prime}\right)_{\Sigma}$ commuting with every element of $S$, or (b) the orbit $\left\{g s g^{-1}: g \in\left(A^{\prime \prime}\right)_{\Sigma}\right\}$ of some $s \in S$ is of size $\geq\left|\left(A^{\prime \prime}\right)_{\Sigma}\right|^{1 / 6} \geq\left(\sigma^{m} m !\right)^{1 / 6}$. This orbit is entirely contained in the pointwise stabilizer $\left(A^{n^{O(\log n)}}\right)_{(\Sigma)}$.

In case (a), $g$ must act trivially on $\Gamma$ (since it commutes with a 2-transitive group on $\Gamma$ ) and so we are done by Corollary 6.4 (Babai, Beals, and Seress). In case (b), we have succeeded in constructing many elements of $A^{n^{O(\log n)}}$ in the pointwise stabilizer of the set $\Sigma$.

This does not mean we are done yet; perhaps there were already many elements of $A^{2}$ in the pointwise stabilizer of $\Sigma$. (Otherwise, Lemma 4.3 does mean that $A$ is growing rapidly, and so we are done.) However, having many elements in the pointwise stabilizer of $\Sigma$ does mean that we can start now an iteration, constructing a second set $\Sigma_{2}$ and a longer stabilizer chain satisfying (6.3) with $A$ replaced by $\left(A^{n^{O(\log n)}}\right)_{(\Sigma)}$, and then a third set $\Sigma_{3}$, and so on. Instead of focusing on making $A$ grow, we focus on making the length of the stabilizer chain grow, until it reaches size about $n$, at which point we are done.

\section{Some OPEN PROBLEMS}

The following questions are hard and far from new.

(a) Consider all Cayley graphs $\Gamma(G, A)$ with $G=\mathrm{SL}_{2}\left(\mathbb{F}_{p}\right), A \subset G,|A|=2$, $\langle A\rangle=G, p$ arbitrary. Are they all $\epsilon$-expanders for some fixed $\epsilon>0$ ?

Here [LW93, p. 96] states that an affirmative answer was made plausible by the experiments in [LR92. The proof in BG08c is valid for $A=A_{0} \bmod p\left(A_{0}\right.$ fixed) and also for $A$ random (with probability 1 ), among other cases; see also [BG10]. Expansion for $A=A_{0} \bmod p, A_{0}$ fixed, is known for all non-abelian simple groups of Lie type $G$ (of bounded rank) thanks to GV12; a proof of expansion has also been announced for such $G$ and $A$ random BGGT. Expansion has been conjectured for general $G$ of bounded rank and arbitrary $A$; see, e.g., [Lub12, Conj. 2.29].

(b) Does every Cayley graph $\Gamma(G, A)$ with $G=\mathrm{SL}_{n}\left(\mathbb{F}_{p}\right), A$ a set of generators of $G$ ( $n$ and $p$ arbitrary) have diameter $(\log |G|)^{C}$, where $C$ is an absolute constant? 
(This is Babai's conjecture in the case of linear algebraic groups. The cases $n=2, n=3$ were proven in [Hel08, Hel11. Both BGT11] and PSa] give this result with $C$ depending on $n$.)

(c) Does every Cayley graph $\Gamma(\operatorname{Sym}(n), A)(A$ a set of generators of $\operatorname{Sym}(n))$ have diameter $n^{O(1)}$ ?

(This predates Babai's more general conjecture. Here Theorem 1.4 (as in [HS14]) is the best result to date.)

(d) Let $A$ consist of two random elements of $\operatorname{Alt}(n)$. Is $\Gamma(\operatorname{Alt}(n), A)$ an $\epsilon$ expander, $\epsilon>0$ fixed? Is the diameter of $\Gamma(\operatorname{Alt}(n), A)$ at most $n(\log n)^{O(1)}$ ?

(A "yes" to the former question implies a "yes" to the latter, but there is no consensus on what the answer to either question should be.)

(e) ("Navigation") Given a set $A$ of generators of $G=\mathrm{SL}_{2}\left(\mathbb{F}_{p}\right)(|A|=2$ if you wish) and an element $g$ of $G$, can you find in time $O\left((\log p)^{c_{1}}\right)$ a product of length $O\left((\log p)^{c_{2}}\right)$ of elements of $A \cup A^{-1}$ equal to $g$ ?

(A probabilistic algorithm for a specific $A$ is given in Lar03.)

One of the difficulties in answering question (ㄷ) resides in the fact that a statement such as Theorem 1.1 cannot be true for all subsets $A$ of a symmetric group $G$. Both Pyber and Spiga have given counterexamples. The following counterexample is due to Pyber. Let $G=\operatorname{Sym}(2 n+1)$ and $A=H \cup\left\{\sigma, \sigma^{-1}\right\}$, where $\sigma$ is the shift $m \rightarrow m+2 \bmod 2 n+1$ and $H$ is the subgroup generated by all transpositions $(i, i+1)$ with $1 \leq i \leq n$. Then $\left|A^{3}\right| \ll|A|$. See also [PPSS12, §3] and [Spi12].

What happens if the conditions on $A \subset \operatorname{Sym}(n)$ are strengthened? Can a statement such as Theorem 1.1 then be true? In a first draft of the present text, the author asked whether $r$-transitivity is enough. That is: let $A \subset G(G=\operatorname{Sym}(n)$ or $G=\operatorname{Alt}(n))$ be a set of generators of $G$; assume that $A$ is $r$-transitive, meaning that, for any two $r$-tuples $v_{1}, v_{2}$ of distinct elements of $\{1,2, \ldots, n\}$, there is a $g \in A$ such that $g$ takes $v_{1}$ to $v_{2}$. If $r$ is greater than a constant (say 6), does it follow that

$$
\text { either } \quad\left|A^{3}\right|>|A|^{1+\delta} \quad \text { or } \quad A^{k}=G \text {, }
$$

where $k$ is an absolute constant? L. Pyber promptly showed that the answer is "no": Let $A$ be the union of any large subgroup $H<G$ and the union of all $2 r$ cycles. Then $\left|A^{3}\right| \leq|C|^{2}|A| \leq n^{4 r}|A|$, and this is much smaller than $|A|^{1+\delta}$ for $H$ large.

What if $A \subset G$ is of the form $A=B^{k}$, where $|B|=O(1)$ ? Is this a sufficient condition for (7.1) to hold? It is easy to see that a "yes" answer here, together with a stronger version of Proposition 6.8 (with $j, h \in A^{n^{O(1)}}$ instead of $j, h \in A^{n^{O(\log n)}}$ ), would imply a "yes" answer to (C) above.

A separate, more open-ended question is that of the relevance of work on permutation groups to the study of linear algebraic groups. As we discussed before, the problem of proving growth in $\mathrm{Alt}_{n}$ is closely related to that of proving growth in $\mathrm{SL}_{n}$ uniformly as $n \rightarrow \infty$.

Challenge. Apply the ideas in HS14 to question (b) above.

Finally, let us end with a question for which the time is arguably ripe, but for which there is still no full answer. The idea is to give a full description of subsets of $A$ that fail to grow. 
Conjecture 1. Let $K$ be a field. Let $A$ be a finite subset of $\mathrm{GL}_{n}(K)$ with $A=A^{-1}$, $e \in A$. Then, for every $R \geq 1$, either

(a) $\left|A^{3}\right| \geq R|A|$; or else

(b) there are two subgroups $H_{1} \leq H_{2}$ in $\mathrm{GL}_{n}(K)$ and an integer $k=O_{n}(1)$ such that

- $H_{1}$ and $H_{2}$ are both normal in $\langle A\rangle$, and $H_{2} / H_{1}$ is nilpotent,

- $A^{k}$ contains $H_{1}$, and

- $\left|A^{k} \cap H_{2}\right| \geq R^{-O_{n}(1)}|A|$.

This conjecture was made fairly explicitly in Hel11 (see comments after Hel11, Thm. 1.1]), where it was also proven for $n=3$ and $K=\mathbb{F}_{p}$ (in a slightly weaker form). The same conjecture was proven for $n$ general and $K=\mathbb{F}_{p}$ as [GH, Thm. 2] (joint with Pyber and Szabó). Breuillard, Green, and Tao have given to this conjecture the name of Helfgott-Lindenstrauss conjecture; in [BGT12], they proved a qualitative version with non-explicit bounds (valid even for non-algebraic groups). The case of $n$ general and $K$ general, as stated here, remains open. A slightly modified version of the conjecture (for $n$ and $K$ general, but with $H_{2} / H_{1}$ soluble rather than nilpotent) has been proven by Pyber and Szabó [PSb, Thm. 8].

\section{ACKNOWLEDGMENTS}

The present paper was written in fulfillment of the conditions for the author's Adams Prize (Cambridge). The author is very grateful to the Universidad Autónoma de Madrid for the opportunity to lecture on the subject during his stay there as a visiting professor; special thanks are due to J. Cilleruelo. Notes taken by students during his previous series of lectures at the AQUA summer school (TIFR, Mumbai, 2010) were also helpful, as was the opportunity to teach at the GANT school in EPFL (Lausanne), and, in the last stages of preparation of the text, at the Analytic Number Theory workshop at IHES. The author would like to thank the organizers in general and E. Kowalski and $\mathrm{Ph}$. Michel in particular.

A special place in the acknowledgments is merited by A. Granville, whose course and whose notes Gra introduced the author to additive combinatorics. E. Kowalski, M. Rudnev, P. Varjú, and E. Vlad must also be thanked profusely for their close reading of large sections of the paper. Many thanks are also due to L. Babai, F. Brumley, N. Gill, B. Green, L. Pyber, P. Spiga, and an anonymous referee for their helpful comments.

\section{ABOut THE AUTHOR}

Harald Helfgott is Directeur de Recherche at CNRS-Paris VI/VII. His interests lie in number theory, group theory, additive combinatorics, and diophantine geometry, among other fields.

\section{REFERENCES}

[AS00] N. Alon and J. H. Spencer, The probabilistic method, 2nd ed., Wiley-Interscience Series in Discrete Mathematics and Optimization, Wiley-Interscience [John Wiley \& Sons], New York, 2000. With an appendix on the life and work of Paul Erdős. MR.1885388 (2003f:60003)

[Bab86] L. Babai, On the length of subgroup chains in the symmetric group, Comm. Algebra 14 (1986), no. 9, 1729-1736, DOI 10.1080/00927878608823393. MR:860123 (87m:20005) 
[Bab06] L. Babai, On the diameter of Eulerian orientations of graphs, Proceedings of the Seventeenth Annual ACM-SIAM Symposium on Discrete Algorithms, ACM, New York, 2006, pp. 822-831, DOI 10.1145/1109557.1109648. MR2368881

[Bab82] L. Babai, On the order of doubly transitive permutation groups, Invent. Math. 65 (1981/82), no. 3, 473-484, DOI 10.1007/BF01396631. MR643565 (83g:20003)

[Bas72] H. Bass, The degree of polynomial growth of finitely generated nilpotent groups, Proc. London Math. Soc. (3) 25 (1972), 603-614. MR0379672 (52 \#577)

[BBS04] L. Babai, R. Beals, and Á. Seress, On the diameter of the symmetric group: polynomial bounds, Proceedings of the Fifteenth Annual ACM-SIAM Symposium on Discrete Algorithms, ACM, New York, 2004, pp. 1108-1112 (electronic). MR2291003

[BD92] D. Bayer and P. Diaconis, Trailing the dovetail shuffle to its lair, Ann. Appl. Probab. 2 (1992), no. 2, 294-313. MR1161056 (93d:60014)

[BG08a] J. Bourgain and A. Gamburd, Expansion and random walks in $\mathrm{SL}_{d}\left(\mathbb{Z} / p^{n} \mathbb{Z}\right)$. I, J. Eur. Math. Soc. (JEMS) 10 (2008), no. 4, 987-1011, DOI 10.4171/JEMS/137. MR2443926 (2010a:05093)

[BG08b] J. Bourgain and A. Gamburd, On the spectral gap for finitely-generated subgroups of SU(2), Invent. Math. 171 (2008), no. 1, 83-121, DOI 10.1007/s00222-007-0072-z. MR2358056 (2009g:22018)

[BG08c] J. Bourgain and A. Gamburd, Uniform expansion bounds for Cayley graphs of $\mathrm{SL}_{2}\left(\mathbb{F}_{p}\right)$, Ann. of Math. (2) 167 (2008), no. 2, 625-642, DOI 10.4007/annals.2008.167.625. MR2415383 (2010b:20070)

[BG09] J. Bourgain and A. Gamburd, Expansion and random walks in $\mathrm{SL}_{d}\left(\mathbb{Z} / p^{n} \mathbb{Z}\right)$. II, J. Eur. Math. Soc. (JEMS) 11 (2009), no. 5, 1057-1103, DOI 10.4171/JEMS/175. With an appendix by Bourgain. MR2538500 (2011a:60021)

[BG10] E. Breuillard and A. Gamburd, Strong uniform expansion in $\mathrm{SL}(2, p)$, Geom. Funct. Anal. 20 (2010), no. 5, 1201-1209, DOI 10.1007/s00039-010-0094-3. MR.2746951 (2011m:20111)

[BG11a] E. Breuillard and B. Green, Approximate groups. I: The torsion-free nilpotent case, J. Inst. Math. Jussieu 10 (2011), no. 1, 37-57, DOI 10.1017/S1474748010000150. MR2749571 (2012b:11014)

[BG11b] E. Breuillard and B. Green, Approximate groups, II: The solvable linear case, Q. J. Math. 62 (2011), no. 3, 513-521, DOI 10.1093/qmath/haq011. MR2825469

[BG12] E. Breuillard and B. Green, Approximate groups III: the unitary case, Turkish J. Math. 36 (2012), no. 2, 199-215. MR2912036

[BGGT] E. Breuillard, B. Green, R. Guralnick, and T. Tao. Expansion in finite simple groups of Lie type. Available as arxiv.org:1309.1975.

$\left[\mathrm{BGH}^{+}{ }^{14}\right]$ J. Bamberg, N. Gill, T. P. Hayes, H. A. Helfgott, Á. Seress, and P. Spiga, Bounds on the diameter of Cayley graphs of the symmetric group, J. Algebraic Combin. 40 (2014), no. 1, 1-22, DOI 10.1007/s10801-013-0476-3. MR3226815

[BGK06] J. Bourgain, A. A. Glibichuk, and S. V. Konyagin, Estimates for the number of sums and products and for exponential sums in fields of prime order, J. London Math. Soc. (2) 73 (2006), no. 2, 380-398, DOI 10.1112/S0024610706022721. MR2225493 (2007e:11092)

[BGS10] J. Bourgain, A. Gamburd, and P. Sarnak, Affine linear sieve, expanders, and sumproduct, Invent. Math. 179 (2010), no. 3, 559-644, DOI 10.1007/s00222-009-0225-3. MR.2587341 (2011d:11018)

[BGS11] J. Bourgain, A. Gamburd, and P. Sarnak, Generalization of Selberg's $\frac{3}{16}$ theorem and affine sieve, Acta Math. 207 (2011), no. 2, 255-290, DOI 10.1007/s11511-012-0070-x. MR2892611

[BGT11] E. Breuillard, B. Green, and T. Tao, Approximate subgroups of linear groups, Geom. Funct. Anal. 21 (2011), no. 4, 774-819, DOI 10.1007/s00039-011-0122-y. MR 2827010

[BGT12] E. Breuillard, B. Green, and T. Tao, The structure of approximate groups, Publ. Math. Inst. Hautes Études Sci. 116 (2012), 115-221, DOI 10.1007/s10240-012-0043-9. MR3090256

[BH92] L. Babai and G. L. Hetyei, On the diameter of random Cayley graphs of the symmetric group, Combin. Probab. Comput. 1 (1992), no. 3, 201-208, DOI 10.1017/S0963548300000237. MR1208801(94b:05095) 
[BH05] L. Babai and T. P. Hayes, Near-independence of permutations and an almost sure polynomial bound on the diameter of the symmetric group, Proceedings of the Sixteenth Annual ACM-SIAM Symposium on Discrete Algorithms, ACM, New York, 2005, pp. 1057-1066 (electronic). MR2298365

[BIW06] B. Barak, R. Impagliazzo, and A. Wigderson, Extracting randomness using few independent sources, SIAM J. Comput. 36 (2006), no. 4, 1095-1118 (electronic), DOI 10.1137/S0097539705447141. MR2272272(2007j:68048)

[BKL89] L. Babai, W. M. Kantor, and A. Lubotsky, Small-diameter Cayley graphs for finite simple groups, European J. Combin. 10 (1989), no. 6, 507-522, DOI 10.1016/S01956698(89)80067-8. MR.1022771 (91a:20038)

[BKT04] J. Bourgain, N. Katz, and T. Tao, A sum-product estimate in finite fields, and applications, Geom. Funct. Anal. 14 (2004), no. 1, 27-57, DOI 10.1007/s00039-004-0451-1. MR2053599(2005d:11028)

[BLS87] L. Babai, E. M. Luks, and Á. Seress. Permutation groups in NC. In Proceedings of the Nineteenth Annual ACM Symposium on the Theory of Computing, pages 409-420, New York, 1987. ACM.

[BNP08] L. Babai, N. Nikolov, and L. Pyber, Product growth and mixing in finite groups, Proceedings of the Nineteenth Annual ACM-SIAM Symposium on Discrete Algorithms, ACM, New York, 2008, pp. 248-257. MR2485310

[Bou72] N. Bourbaki, Éléments de mathématique. Fasc. XXXVII. Groupes et algèbres de Lie. Chapitre II: Algèbres de Lie libres. Chapitre III: Groupes de Lie, Hermann, Paris, 1972. Actualités Scientifiques et Industrielles, No. 1349. Mr.0573068 (58 \#28083a)

[BRD] J. Button and C. Roney-Dougal, An explicit upper bound for the Helfgott delta in SL(2,p), J. Algebra 421 (2015), 493-511. MR3272393

[Bro86] R. Brooks, The spectral geometry of a tower of coverings, J. Differential Geom. 23 (1986), no. 1, 97-107. MR840402 (87j:58095)

[Bro87] R. Brooks, On the angles between certain arithmetically defined subspaces of $\mathbf{C}^{n}$ (English, with French summary), Ann. Inst. Fourier (Grenoble) 37 (1987), no. 1, 175185. MR894565 (89h:11022)

[BS87a] L. Babai and Á. Seress, On the degree of transitivity of permutation groups: a short proof, J. Combin. Theory Ser. A 45 (1987), no. 2, 310-315, DOI 10.1016/00973165(87)90023-9. MR894827 (88h:20002)

[BS87b] A. Broder and E. Shamir. On the second eigenvalue of random regular graphs. In 28th Annual Symposium on the Foundations of Computer Science (FOCS 1987), 1987.

[BS88] L. Babai and Á. Seress, On the diameter of Cayley graphs of the symmetric group, J. Combin. Theory Ser. A 49 (1988), no. 1, 175-179, DOI 10.1016/0097-3165(88)90033-7. MR957215 (89i:05141)

[BS92] L. Babai and Á. Seress, On the diameter of permutation groups, European J. Combin. 13 (1992), no. 4, 231-243, DOI 10.1016/S0195-6698(05)80029-0. MR.1179520 (93h:20001)

[BS94] A. Balog and E. Szemerédi, A statistical theorem of set addition, Combinatorica 14 (1994), no. 3, 263-268, DOI 10.1007/BF01212974. MR1305895(95m:11019)

[Bur86] M. Burger. Petites valeurs propres du Laplacien et topologie de Fell. PhD thesis, Université de Lausanne, 1986.

[Bus78] P. Buser, Cubic graphs and the first eigenvalue of a Riemann surface, Math. Z. 162 (1978), no. 1, 87-99, DOI 10.1007/BF01437826. MR505920 (80b:58061)

[BV12] J. Bourgain and P. P. Varjú, Expansion in $S L_{d}(\mathbf{Z} / q \mathbf{Z}), q$ arbitrary, Invent. Math. 188 (2012), no. 1, 151-173, DOI 10.1007/s00222-011-0345-4. MR2897695

[Cha02] M.-C. Chang, A polynomial bound in Freiman's theorem, Duke Math. J. 113 (2002), no. 3, 399-419, DOI 10.1215/S0012-7094-02-11331-3. MR.1909605 (2003d:11151)

[Cha08] M.-C. Chang, Product theorems in $\mathrm{SL}_{2}$ and $\mathrm{SL}_{3}$, J. Inst. Math. Jussieu 7 (2008), no. 1, 1-25, DOI 10.1017/S1474748007000126. MR2398145 (2009d:20112)

[CS10] E. Croot and O. Sisask, A probabilistic technique for finding almost-periods of convolutions, Geom. Funct. Anal. 20 (2010), no. 6, 1367-1396, DOI 10.1007/s00039-0100101-8. MR2738997 (2012d:11019)

[Din06] O. Dinai, Poly-log diameter bounds for some families of finite groups, Proc. Amer. Math. Soc. 134 (2006), no. 11, 3137-3142 (electronic), DOI 10.1090/S0002-9939-0608384-5. MR2231895(2007j:05097) 
[Din11] O. Dinai, Growth in $\mathrm{SL}_{2}$ over finite fields, J. Group Theory 14 (2011), no. 2, 273-297, DOI 10.1515/JGT.2010.056. MR2788087 (2012c:20134)

[Dix69] J. D. Dixon, The probability of generating the symmetric group, Math. Z. 110 (1969), 199-205. MR0251758(40 \#4985)

[DM96] J. D. Dixon and B. Mortimer, Permutation groups, Graduate Texts in Mathematics, vol. 163, Springer-Verlag, New York, 1996. MR.1409812 (98m:20003)

[DS81] P. Diaconis and M. Shahshahani, Generating a random permutation with random transpositions, Z. Wahrsch. Verw. Gebiete 57 (1981), no. 2, 159-179, DOI 10.1007/BF00535487. MR626813 (82h:60024)

[DS98] V. I. Danilov and V. V. Shokurov, Algebraic curves, algebraic manifolds and schemes, Springer-Verlag, Berlin, 1998. Translated from the 1988 Russian original by D. Coray and V. N. Shokurov; Translation edited and with an introduction by I. R. Shafarevich; Reprint of the original English edition from the series Encyclopaedia of Mathematical Sciences [Algebraic geometry. I, Encyclopaedia Math. Sci., 23, Springer, Berlin, 1994; MR1287418 (95b:14001)]. MR1658464 (99g:14001)

[DSC93] P. Diaconis and L. Saloff-Coste, Comparison techniques for random walk on finite groups, Ann. Probab. 21 (1993), no. 4, 2131-2156. MR1245303 (95a:60009)

[DSV03] G. Davidoff, P. Sarnak, and A. Valette, Elementary number theory, group theory, and Ramanujan graphs, London Mathematical Society Student Texts, vol. 55, Cambridge University Press, Cambridge, 2003. MR.1989434 (2004f:11001)

[EHK12] J. S. Ellenberg, C. Hall, and E. Kowalski, Expander graphs, gonality, and variation of Galois representations, Duke Math. J. 161 (2012), no. 7, 1233-1275, DOI 10.1215/00127094-1593272. MR.2922374

[EK01] G. Elekes and Z. Király, On the combinatorics of projective mappings, J. Algebraic Combin. 14 (2001), no. 3, 183-197, DOI 10.1023/A:1012799318591. MR.1869409 (2003e:52034)

[Ele97] G. Elekes, On the number of sums and products, Acta Arith. 81 (1997), no. 4, 365-367. MR.1472816 (98h:11026)

[EM03] G. A. Edgar and C. Miller, Borel subrings of the reals, Proc. Amer. Math. Soc. 131 (2003), no. 4, 1121-1129 (electronic), DOI 10.1090/S0002-9939-02-06653-4. MR1948103 (2004d:28017)

[EMO05] A. Eskin, S. Mozes, and H. Oh, On uniform exponential growth for linear groups, Invent. Math. 160 (2005), no. 1, 1-30, DOI 10.1007/s00222-004-0378-z. MR2129706 (2006a:20081)

[ES83] P. Erdős and E. Szemerédi, On sums and products of integers, Studies in pure mathematics, Birkhäuser, Basel, 1983, pp. 213-218. MR820223 (86m:11011)

[FH91] W. Fulton and J. Harris, Representation theory, Graduate Texts in Mathematics, vol. 129, Springer-Verlag, New York, 1991. A first course; Readings in Mathematics. MR.1153249 (93a:20069)

[Fiz] G. Fiz Pontiveros, Sums of dilates in $\mathbb{Z}_{p}$, Combin. Probab. Comput. 22 (2013), no. 2, 282-293, DOI 10.1017/S0963548312000466. MR3021335

[FKP10] D. Fisher, N. H. Katz, and I. Peng, Approximate multiplicative groups in nilpotent Lie groups, Proc. Amer. Math. Soc. 138 (2010), no. 5, 1575-1580, DOI 10.1090/S00029939-10-10078-1. MR2587441(2011e:11164)

[Fre73] G. A. Freimman, Foundations of a structural theory of set addition, American Mathematical Society, Providence, R. I., 1973. Translated from the Russian; Translations of Mathematical Monographs, Vol 37. MR0360496 (50 \#12944)

[Fur77] H. Furstenberg, Ergodic behavior of diagonal measures and a theorem of Szemerédi on arithmetic progressions, J. Analyse Math. 31 (1977), 204-256. MR.0498471 (58 \#16583)

[Gam02] A. Gamburd, On the spectral gap for infinite index "congruence" subgroups of $\mathrm{SL}_{2}$ (Z), Israel J. Math. 127 (2002), 157-200, DOI 10.1007/BF02784530. MR.1900698 (2003b:11050)

[GH] N. Gill and H. Helfgott, Growth in solvable subgroups of $\mathrm{GL}_{r}(\mathbb{Z} / p \mathbb{Z})$, Math. Annalen 360 (2014), no. 1-2, 157-208. MR3263161

[GH11] N. Gill and H. A. Helfgott, Growth of small generating sets in $\mathrm{SL}_{n}(\mathbb{Z} / p \mathbb{Z})$, Int. Math. Res. Not. IMRN 18 (2011), 4226-4251. MR.2836020 
[GHR] N. Gill, H. Helfgott, and M. Rudnev. On growth in an abstract plane. To appear in Proc. Amer. Math. Soc. Available as arxiv:1212.5056.

[GK07] A. A. Glibichuk and S. V. Konyagin, Additive properties of product sets in fields of prime order, Additive combinatorics, CRM Proc. Lecture Notes, vol. 43, Amer. Math. Soc., Providence, RI, 2007, pp. 279-286. MR2359478 (2009a:11054)

[Gow98] W. T. Gowers, A new proof of Szemerédi's theorem for arithmetic progressions of length four, Geom. Funct. Anal. 8 (1998), no. 3, 529-551, DOI 10.1007/s000390050065. MR.1631259(2000d:11019)

[Gow01] W. T. Gowers, A new proof of Szemerédi's theorem, Geom. Funct. Anal. 11 (2001), no. 3, 465-588, DOI 10.1007/s00039-001-0332-9. MR.1844079(2002k:11014)

[Gow08] W. T. Gowers, Quasirandom groups, Combin. Probab. Comput. 17 (2008), no. 3, 363-387, DOI 10.1017/S0963548307008826. MR2410393 (2009f:20105)

[GR05] B. Green and I. Z. Ruzsa, Sum-free sets in abelian groups, Israel J. Math. 147 (2005), 157-188, DOI 10.1007/BF02785363. MR2166359 (2006e:11030)

[Gra] A. Granville. Additive combinatorics. Unpublished notes.

[Gro81] M. Gromov, Groups of polynomial growth and expanding maps, Inst. Hautes Études Sci. Publ. Math. 53 (1981), 53-73. MR623534 (83b:53041)

[GS04] A. Gamburd and M. Shahshahani, Uniform diameter bounds for some families of Cayley graphs, Int. Math. Res. Not. 71 (2004), 3813-3824, DOI 10.1155/S1073792804140579. MR2104475 (2005m:05111)

[GT08] B. Green and T. Tao, The primes contain arbitrarily long arithmetic progressions, Ann. of Math. (2) 167 (2008), no. 2, 481-547, DOI 10.4007/annals.2008.167.481. MR 2415379 (2009e:11181)

[Gui73] Y. Guivarc'h, Croissance polynomiale et périodes des fonctions harmoniques (French), Bull. Soc. Math. France 101 (1973), 333-379. MR0369608 (51 \#5841)

[GV12] A. S. Golsefidy and P. P. Varjú, Expansion in perfect groups, Geom. Funct. Anal. 22 (2012), no. 6, 1832-1891, DOI 10.1007/s00039-012-0190-7. MR3000503

[Hel08] H. A. Helfgott, Growth and generation in $\mathrm{SL}_{2}(\mathbb{Z} / p \mathbb{Z})$, Ann. of Math. (2) 167 (2008), no. 2, 601-623, DOI 10.4007/annals.2008.167.601. MR2415382(2009i:20094)

[Hel11] H. A. Helfgott, Growth in $\mathrm{SL}_{3}(\mathbb{Z} / p \mathbb{Z})$, J. Eur. Math. Soc. (JEMS) 13 (2011), no. 3, 761-851, DOI 10.4171/JEMS/267. MR2781932

[HK05] B. Host and B. Kra, Nonconventional ergodic averages and nilmanifolds, Ann. of Math. (2) 161 (2005), no. 1, 397-488, DOI 10.4007/annals.2005.161.397. MR2150389 (2007b:37004)

[HP95] E. Hrushovski and A. Pillay, Definable subgroups of algebraic groups over finite fields, J. Reine Angew. Math. 462 (1995), 69-91. MR1329903 (97f:20059)

[Hru12] E. Hrushovski, Stable group theory and approximate subgroups, J. Amer. Math. Soc. 25 (2012), no. 1, 189-243, DOI 10.1090/S0894-0347-2011-00708-X. MR 2833482 (2012h:03104)

[HS14] H. A. Helfgott and Á. Seress, On the diameter of permutation groups, Ann. of Math. (2) 179 (2014), no. 2, 611-658, DOI 10.4007/annals.2014.179.2.4. MR3152942

[HSZ] H. Helfgott, Á. Seress, and A. Zuk, Random generators of the symmetric group: diameter, mixing time and spectral gap, J. Algebra 421 (2015), 349-368. MR.3272386

[HW08] E. Hrushovski and F. Wagner, Counting and dimensions, Model theory with applications to algebra and analysis. Vol. 2, London Math. Soc. Lecture Note Ser., vol. 350, Cambridge Univ. Press, Cambridge, 2008, pp. 161-176, DOI 10.1017/CBO9780511735219.005. MR2436141 (2009k:03042)

[Kas07] M. Kassabov, Symmetric groups and expander graphs, Invent. Math. 170 (2007), no. 2, 327-354, DOI 10.1007/s00222-007-0065-y. MR2342639 (2008g:20009)

[Kaž67] D. A. Každan, On the connection of the dual space of a group with the structure of its closed subgroups (Russian), Funkcional. Anal. i Priložen. 1 (1967), 71-74. MR.0209390 (35 \#288)

[Kes59] H. Kesten, Symmetric random walks on groups, Trans. Amer. Math. Soc. 92 (1959), 336-354. MR0109367 (22 \#253)

[KMS84] D. Kornhauser, G. Miller, and P. Spirakis. Coordinating pebble motion on graphs, the diameter of permutation groups, and applications. In Proceedings of the 25th IEEE Symposium on Foundations of Computer Science, pages 241-250, Singer Island, FL, 1984. IEEE Computer Society Press. 
[Kon92] S. V. Konyagin, Estimates for Gaussian sums and Waring's problem modulo a prime (Russian), Trudy Mat. Inst. Steklov. 198 (1992), 111-124; English transl., Proc. Steklov Inst. Math. 1 (198) (1994), 105-117. MR1289921 (96e:11122)

[Kowa] E. Kowalski. Expander graphs. Course notes available at http://www.math.ethz.ch/ kowalski/expander-graphs.pdf.

[Kowb] E. Kowalski. Sieve in expansion. Séminaire Bourbaki, 63ème année, 2010-2011, no. 1028.

[Kow13] E. Kowalski, Explicit growth and expansion for $\mathrm{SL}_{2}$, Int. Math. Res. Not. IMRN 24 (2013), 5645-5708. MR3144176

[Lar03] M. Larsen, Navigating the Cayley graph of $\mathrm{SL}_{2}\left(\mathbb{F}_{p}\right)$, Int. Math. Res. Not. 27 (2003), 1465-1471, DOI 10.1155/S1073792803130383. MR1976231 (2004e:22013)

[Lor12] O. Lorscheid, Algebraic groups over the field with one element, Math. Z. 271 (2012), no. 1-2, 117-138, DOI 10.1007/s00209-011-0855-1. MR2917136

[Lov96] L. Lovász, Random walks on graphs: a survey, Combinatorics, Paul Erdős is eighty, Vol. 2 (Keszthely, 1993), Bolyai Soc. Math. Stud., vol. 2, János Bolyai Math. Soc., Budapest, 1996, pp. 353-397. MR1395866 (97a:60097)

[LP11] M. J. Larsen and R. Pink, Finite subgroups of algebraic groups, J. Amer. Math. Soc. 24 (2011), no. 4, 1105-1158, DOI 10.1090/S0894-0347-2011-00695-4. MR.2813339 (2012f:20148)

[LPS88] A. Lubotzky, R. Phillips, and P. Sarnak, Ramanujan graphs, Combinatorica 8 (1988), no. 3, 261-277, DOI 10.1007/BF02126799. MR.963118(89m:05099)

[LPW09] D. A. Levin, Y. Peres, and E. L. Wilmer, Markov chains and mixing times, American Mathematical Society, Providence, RI, 2009. With a chapter by James G. Propp and David B. Wilson. MR2466937 (2010c:60209)

[LR92] J. D. Lafferty and D. Rockmore, Fast Fourier analysis for $\mathrm{SL}_{2}$ over a finite field and related numerical experiments, Experiment. Math. 1 (1992), no. 2, 115-139. MR.1203870 (94f:11131)

[LS74] V. Landazuri and G. M. Seitz, On the minimal degrees of projective representations of the finite Chevalley groups, J. Algebra 32 (1974), 418-443. MR0360852 (50 \#13299)

[Lub12] A. Lubotzky, Expander graphs in pure and applied mathematics, Bull. Amer. Math. Soc. (N.S.) 49 (2012), no. 1, 113-162, DOI 10.1090/S0273-0979-2011-013593. MR2869010 (2012m:05003)

[LW54] S. Lang and A. Weil, Number of points of varieties in finite fields, Amer. J. Math. 76 (1954), 819-827. MR0065218 (16,398d)

[LW93] A. Lubotzky and B. Weiss, Groups and expanders, Expanding graphs (Princeton, NJ, 1992), DIMACS Ser. Discrete Math. Theoret. Comput. Sci., vol. 10, Amer. Math. Soc., Providence, RI, 1993, pp. 95-109. MR1235570 (95b:05097)

[McK84] P. McKenzie, Permutations of bounded degree generate groups of polynomial diameter, Inform. Process. Lett. 19 (1984), no. 5, 253-254, DOI 10.1016/0020-0190(84)90062-0. MR.777808 (86h:68077)

[Mil68] J. Milnor, Growth of finitely generated solvable groups, J. Differential Geometry 2 (1968), 447-449. MR0244899 (39 \#6212)

[MV00] F. Martin and A. Valette, Markov operators on the solvable Baumslag-Solitar groups, Experiment. Math. 9 (2000), no. 2, 291-300. MR1780213 (2002a:37005)

[MVW84] C. R. Matthews, L. N. Vaserstein, and B. Weisfeiler, Congruence properties of Zariskidense subgroups. I, Proc. London Math. Soc. (3) 48 (1984), no. 3, 514-532, DOI 10.1112/plms/s3-48.3.514. MR735226 (85d:20040)

[NC00] M. A. Nielsen and I. L. Chuang, Quantum computation and quantum information, Cambridge University Press, Cambridge, 2000. MR.1796805 (2003j:81038)

[Nor87] M. V. Nori, On subgroups of $\mathrm{GL}_{n}\left(\mathbf{F}_{p}\right)$, Invent. Math. 88 (1987), no. 2, 257-275, DOI 10.1007/BF01388909. MR880952 (88d:20068)

[NP11] N. Nikolov and L. Pyber, Product decompositions of quasirandom groups and a Jordan type theorem, J. Eur. Math. Soc. (JEMS) 13 (2011), no. 4, 1063-1077, DOI 10.4171/JEMS/275. MR2800484 (2012h:20054)

[Pet12] G. Petridis, New proofs of Plünnecke-type estimates for product sets in groups, Combinatorica 32 (2012), no. 6, 721-733, DOI 10.1007/s00493-012-2818-5. MR3063158

[Plü70] H. Plünnecke, Eine zahlentheoretische Anwendung der Graphentheorie (German), J. Reine Angew. Math. 243 (1970), 171-183. MR0266892(42 \#1794) 
[PPSS12] C. E. Praeger, L. Pyber, P. Spiga, and E. Szabó, Graphs with automorphism groups admitting composition factors of bounded rank, Proc. Amer. Math. Soc. 140 (2012), no. 7, 2307-2318, DOI 10.1090/S0002-9939-2011-11100-6. MR2898694

[PSa] L. Pyber and E. Szabó. Growth in finite simple groups of Lie type of bounded rank. Submitted. Available at arxiv.org:1005.1881.

[PSb] L. Pyber and E. Szabó, Growth in linear groups: thin groups and superstrong approximation, Math. Res. Sci. Inst. Publ., vol. 61, Cambridge University Press, Cambridge, 2014. MR3220893

[Pyb93] L. Pyber, On the orders of doubly transitive permutation groups, elementary estimates, J. Combin. Theory Ser. A 62 (1993), no. 2, 361-366, DOI 10.1016/0097-3165(93)90053B. MR $1207742(94 \mathrm{~d}: 20005)$

[Raz14] A. A. Razborov, A product theorem in free groups, Ann. of Math. (2) 179 (2014), no. 2, 405-429, DOI 10.4007/annals.2014.179.2.1. MR 3152939

[Rok63] V. A. Rohlin, Generators in ergodic theory (Russian, with English summary), Vestnik Leningrad. Univ. 18 (1963), no. 1, 26-32. MR0193206 (33 \#1427)

[Rot53] K. F. Roth, On certain sets of integers, J. London Math. Soc. 28 (1953), 104-109. MR.0051853 (14,536g)

[RT85] I. Z. Ruzsa and S. Turjányi, A note on additive bases of integers, Publ. Math. Debrecen 32 (1985), no. 1-2, 101-104. MR810596 (87a:11014)

[Ruz89] I. Z. Ruzsa, An application of graph theory to additive number theory, Sci. Ser. A Math. Sci. (N.S.) 3 (1989), 97-109. MR2314377

[Ruz91] I. Z. Ruzsa, Arithmetic progressions in sumsets, Acta Arith. 60 (1991), no. 2, 191-202. MR.1139055 (92k:11009)

[Ruz94] I. Z. Ruzsa, Generalized arithmetical progressions and sumsets, Acta Math. Hungar. 65 (1994), no. 4, 379-388, DOI 10.1007/BF01876039. MR1281447 (95k:11011)

[Ruz99] I. Z. Ruzsa, An analog of Freiman's theorem in groups (English, with English and French summaries), Astérisque 258 (1999), xv, 323-326. Structure theory of set addition. MR1701207 (2000h:11111)

[San12] T. Sanders, On the Bogolyubov-Ruzsa lemma, Anal. PDE 5 (2012), no. 3, 627-655, DOI 10.2140/apde.2012.5.627. MR2994508

[Sel65] A. Selberg, On the estimation of Fourier coefficients of modular forms, Proc. Sympos. Pure Math., Vol. VIII, Amer. Math. Soc., Providence, R.I., 1965, pp. 1-15. MR.0182610 $(32 \# 93)$

[Ser03] Á. Seress, Permutation group algorithms, Cambridge Tracts in Mathematics, vol. 152, Cambridge University Press, Cambridge, 2003. MR1970241 (2004c:20008)

[Sha97] Y. Shalom, Expanding graphs and invariant means, Combinatorica 17 (1997), no. 4, 555-575, DOI 10.1007/BF01195004. MR1645694 (99h:05057)

[Sha99] Y.Shalom, Expander graphs and amenable quotients, Emerging applications of number theory (Minneapolis, MN, 1996), IMA Vol. Math. Appl., vol. 109, Springer, New York, 1999, pp. 571-581, DOI 10.1007/978-1-4612-1544-8_23. MR 1691549 (2000h:20059)

[Sim70] C. C. Sims, Computational methods in the study of permutation groups, Computational Problems in Abstract Algebra (Proc. Conf., Oxford, 1967), Pergamon, Oxford, 1970, pp. 169-183. MR0257203 (41 \#1856)

[Sim71] C. C. Sims. Computation with permutation groups. In Proc. Second Symposium on Symbolic and Algebraic Maniupulation, pages 23-28. ACM Press, New York, NY, 1971.

[Sol09] J. Solymosi, Bounding multiplicative energy by the sumset, Adv. Math. 222 (2009), no. 2, 402-408, DOI 10.1016/j.aim.2009.04.006. MR2538014(2010h:11014)

[SP12] J.-C. Schlage-Puchta, Applications of character estimates to statistical problems for the symmetric group, Combinatorica 32 (2012), no. 3, 309-323, DOI 10.1007/s00493012-2502-9. MR2965280

[Spi12] P. Spiga, Two local conditions on the vertex stabiliser of arc-transitive graphs and their effect on the Sylow subgroups, J. Group Theory 15 (2012), no. 1, 23-35, DOI 10.1515/JGT.2011.097. MR2876252

[Spr86] T. A. Springer, Conjugacy classes in algebraic groups, Group theory, Beijing 1984, Lecture Notes in Math., vol. 1185, Springer, Berlin, 1986, pp. 175-209, DOI 10.1007/BFb0076175. MR842444 (87i:20082) 
[SSV05] B. Sudakov, E. Szemerédi, and V. H. Vu, On a question of Erdös and Moser, Duke Math. J. 129 (2005), no. 1, 129-155, DOI 10.1215/S0012-7094-04-12915-X. MR2155059 (2006c:11118)

[ST83] E. Szemerédi and W. T. Trotter Jr., Extremal problems in discrete geometry, Combinatorica 3 (1983), no. 3-4, 381-392, DOI 10.1007/BF02579194. MR729791(85j:52014)

[SX91] P. Sarnak and X. X. Xue, Bounds for multiplicities of automorphic representations, Duke Math. J. 64 (1991), no. 1, 207-227, DOI 10.1215/S0012-7094-91-06410-0. MR:1131400(92h:22026)

[Sze] B. Szegedy. On higher order Fourier analysis. Available as arxiv.org:1203.2260.

[Sze69] E. Szemerédi, On sets of integers containing no four elements in arithmetic progression, Acta Math. Acad. Sci. Hungar. 20 (1969), 89-104. MR0245555 (39 \#6861)

[Tao] T. Tao. Course notes. Available at http://www.math.ucla.edu/ tao/254b.1.12w/.

[Tao08] T. Tao, Product set estimates for non-commutative groups, Combinatorica 28 (2008), no. 5, 547-594, DOI 10.1007/s00493-008-2271-7. MR2501249(2010b:11017)

[Tao10] T. Tao, Freiman's theorem for solvable groups, Contrib. Discrete Math. 5 (2010), no. 2, 137-184. MR2791295 (2012e:05063)

[Tit57] J. Tits, Sur les analogues algébriques des groupes semi-simples complexes (French), Colloque d'algèbre supérieure, tenu à Bruxelles du 19 au 22 décembre 1956, Centre Belge de Recherches Mathématiques, Établissements Ceuterick, Louvain; Librairie Gauthier-Villars, Paris, 1957, pp. 261-289. MR.0108765 (21 \#7477)

[Tit72] J. Tits, Free subgroups in linear groups, J. Algebra 20 (1972), 250-270. MR.0286898 (44 \#4105)

[Toi] M. Tointon. Freiman's theorem in an arbitrary nilpotent group. Submitted. Available as arxiv:1211.3989.

[TV06] T. Tao and V. Vu, Additive combinatorics, Cambridge Studies in Advanced Mathematics, vol. 105, Cambridge University Press, Cambridge, 2006. MR2289012 (2008a:11002)

[Var] P. P. Varjú, Random walks in compact groups, Doc. Math. 18 (2013), 1137-1175. MR.3138842

[Var12] P. P. Varjú, Expansion in $S L_{d}\left(\mathcal{O}_{K} / I\right)$, I square-free, J. Eur. Math. Soc. (JEMS) 14 (2012), no. 1, 273-305, DOI 10.4171/JEMS/302. MR2862040

[Wei84] B. Weisfeiler, Strong approximation for Zariski-dense subgroups of semisimple algebraic groups, Ann. of Math. (2) 120 (1984), no. 2, 271-315, DOI 10.2307/2006943. MR:763908 (86m:20053)

[Wie64] H. Wielandt, Finite permutation groups, Translated from the German by R. Bercov, Academic Press, New York-London, 1964. MR0183775 (32 \#1252)

[Wol68] J. A. Wolf, Growth of finitely generated solvable groups and curvature of Riemanniann manifolds, J. Differential Geometry 2 (1968), 421-446. MR.0248688 (40 \#1939)

[Zie07] T. Ziegler, Universal characteristic factors and Furstenberg averages, J. Amer. Math. Soc. 20 (2007), no. 1, 53-97 (electronic), DOI 10.1090/S0894-0347-06-005327. MR2257397 (2007j:37004)

École Normale Supérieure, Département de Mathématiques, 45 Rue D’Ulm, F-75230 PARIS, FRANCE

E-mail address: harald.helfgott@ens.fr 